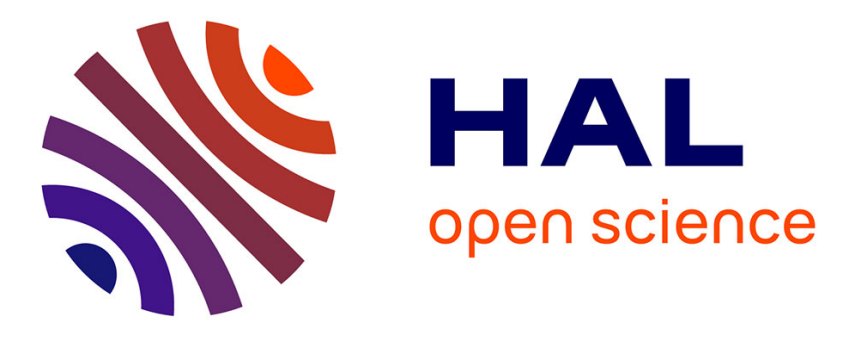

\title{
Application of Quantitative 1H and 19F NMR to Organometallics
}

Ayman Akhdar, Jean-Michel Andanson, Sophie Faure, Mounir Traïkia, Arnaud Gautier

\section{- To cite this version:}

Ayman Akhdar, Jean-Michel Andanson, Sophie Faure, Mounir Traïkia, Arnaud Gautier. Application of Quantitative 1H and 19F NMR to Organometallics. Journal of Organometallic Chemistry, 2021, 950, pp.121991. 10.1016/j.jorganchem.2021.121991 . hal-03368939

\section{HAL Id: hal-03368939 \\ https://hal.uca.fr/hal-03368939}

Submitted on 13 Oct 2021

HAL is a multi-disciplinary open access archive for the deposit and dissemination of scientific research documents, whether they are published or not. The documents may come from teaching and research institutions in France or abroad, or from public or private research centers.
L'archive ouverte pluridisciplinaire HAL, est destinée au dépôt et à la diffusion de documents scientifiques de niveau recherche, publiés ou non, émanant des établissements d'enseignement et de recherche français ou étrangers, des laboratoires publics ou privés. 


\title{
Application of Quantitative ${ }^{1} \mathrm{H}$ and ${ }^{19} \mathrm{~F}$ NMR to Organometallics
}

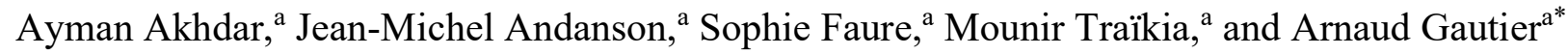
CNRS-UMR 6296, Université Clermont Auvergne, ICCF, F-63000 Clermont-Ferrand, France.

* arnaud.gautier@uca.fr

\begin{abstract}
Purity assessment of organometallics is particularly important for catalytic applications. While quantitative NMR is a well-known method in pharmaceutic chemistry, the present work illustrates its usefulness for the determination of the ligands and organometallics purities using proton and fluorine NMR. This method is fast, straightforward and provides accuracy results.
\end{abstract}

\section{Keywords.}

Purity, NMR, Analytic Chemistry, Imidazolium, Organometallics. 


\section{Introduction.}

The structure determination of ligands and organometallic complexes is usually performed by NMR, infrared (IR), Ultraviolet-Visible (UV-vis) and X-ray spectroscopies. The purity is determined accurately by HPLC for ligands if analytical pure samples of the constituent(s) of a mixture are available. However, this technique cannot be applied routinely to organometallic complexes. Elemental analysis is useful to establish elemental composition and molecular formula. However, it does not provide a direct measurement of the molar purity. Quantitative NMR (qNMR) presents a valuable alternative because the measured signals represent a direct measurement of the composition. [1] Therefore, in presence of an internal reference of known purity, the composition of a synthetic sample can be determined accurately using a single spectrum/ integration sequence (so-called absolute quantification). Equation 1 affords the purity value after accurate weight and NMR measurements.

$P_{s}=\frac{I_{s}}{I_{s t d}} \cdot \frac{N_{s t d}}{N_{s}} \cdot \frac{M_{s}}{M_{s t d}} \cdot \frac{m_{s t d}}{m_{s}} \cdot P_{s t d}$ Eq. 1

(where $\mathrm{s}$ and std stand for the sample and the standard respectively; I being the sum of integrals, $\mathrm{N}$ the total number of nucleus $\left({ }^{1} \mathrm{H},{ }^{19} \mathrm{~F}\right.$ for example), $\mathrm{M}$ the molecular mass, $\mathrm{m}$ the measured masses, and $\mathrm{P}$ the purities of the standard). This method gives a direct access to the sample purity regardless the content of water or residual solvents, inorganic or organic chemicals, and sorbents, such as silica gel or celite. qNMR, especially using proton measurement is now a technique of choice routinely used in natural product and pharmaceutical chemistry with a reported accuracy of 1-2\%. [1a] Several reviews are available, [1b] 
reporting the acquisition parameters and this protocol is recently entered into the guideline for authors of the Journal of Medicinal Chemistry.[2] Chemical suppliers are commercializing 'gold standard' which are used as internal or external standards. Expensive 'gold standards' can also be used to determine the purity of a cheaper or more adequate secondary standard, especially if the commercial standard is not compatible with the chemical stability of the organometallic complexes. The purity is of importance for the synthesis of the desired complexes. For example, the starting material could contain residual water, a metathesis could afford a mixture of counter ions or a transmetallation leading to a contamination. In addition, in the case of kinetic studies the determination of the concentrations in stock solutions (reactants and catalyst) should be taken into consideration to perform accurate measurements. In this communication, the application of ${ }^{1} \mathrm{H}$ and ${ }^{19} \mathrm{~F}$ qNMR for ligands and organometallics is reported (ESI for NMR parameters and protocols). In brief, after proper weight measurements, choice of the adequate solvent, recording of ${ }^{1} \mathrm{H}$ and ${ }^{19} \mathrm{~F}$ spectrum takes 30 to 60 minutes making this technic attractive for routine determination.

\section{Results and discussion.}

\subsection{Choice of the standard.}

To make this method straightforward and broadly applicable to a wide variety of ligands and organometallics, the standard should be cheap and inert in presence of the organometallics. This prohibits standards containing some functional groups that could chelate or react such as carbonyls, reducing agents (formic salts), double bonds, acidic or redox compounds (phenol, quinones). We find that 1,4-dimethoxy 
benzene $(1,4-D M B)$ is a good secondary standard for ${ }^{1} \mathrm{H}$ qNMR as it is cheap, easily purified by recrystallization from hexane or sublimation [3] and presents a good solubility in all common NMR solvents. Using maleic acid as primary gold standard (99.995\% traceable certified reference materials, TraceCERT ${ }^{\oplus}$ ) we find a purity of $99.5 \%$ for our recrystallized sample of 1,4-DMB (Karl Fisher analysis demonstrates the absence of residual water). ${ }^{1} \mathrm{H}$ NMR of $1,4-\mathrm{DMB}$ shows two well defined, sharp singlets at 6.7 and $3.7 \mathrm{ppm}$. Regarding ${ }^{19} \mathrm{~F}$ qNMR, we used the commercially available 'gold standard' 2-chloro-4fluoro toluene that is found inert in presence of the compounds described below. It is also interesting to note that the chemical shift of this standard is well positioned between the signals displayed by $\mathrm{BF}_{4}$ and $\mathrm{PF}_{6}$ anions. $\mathrm{PF}_{6}$ appears as a doublet due to the coupling with phosphorus whereas $\mathrm{BF}_{4}$ appears as two close signals, a singlet $\left({ }^{10} \mathrm{~B}\right)$ and a quadruplet $\left({ }^{11} \mathrm{~B}, \mathrm{~S}=3 / 2, \mathrm{~J}=1.1 \mathrm{~Hz}\right)$ in a ratio $2: 8$. Chemical shifts $(\delta)$ and relaxation times (T1) are collected in Table 1. The relaxation times show that 30 seconds of inter-pulse delay (>5 T1) could be used for ${ }^{19} \mathrm{~F}$ qNMR.

Table 1: Chemical shifts $(\delta)$ and relaxation times (T1)

\begin{tabular}{|c|c|c|c|}
\hline compound & Solvent & $\delta(\mathrm{ppm})$ & T1 (s) \\
\hline $\begin{array}{l}\text { 2-chloro-4-fluoro } \\
\text { Toluene }\end{array}$ & $\begin{array}{l}\text { dmso-d } 6 \\
\text { Acetone-d } 6 \\
\text { MeOH-d }_{4} \\
{\mathrm{MeCN}-\mathrm{d}_{3}} \\
\mathrm{CDCl}_{3}\end{array}$ & $\begin{array}{l}-115.3 \\
-117.0 \\
-117.7 \\
-117.3 \\
-115.8\end{array}$ & $\begin{array}{l}3.3^{[\mathrm{a}]} \\
6.5^{[\mathrm{b}]} \\
4.8^{[\mathrm{a}]} \\
4.7^{[\mathrm{a}]} \\
4.4^{[\mathrm{a}]}\end{array}$ \\
\hline $\mathrm{BF}_{4}^{-}$ & $\begin{array}{l}\text { dmso-d } 6 \\
\text { Acetone-d } 6 \\
\text { MeOH-d }_{4} \\
\mathrm{MeCN}_{3} \mathrm{~d}_{3} \\
\mathrm{CDCl}_{3}\end{array}$ & \begin{tabular}{|l|}
-148.3 \\
-151.8 \\
-154.8 \\
-151.8 \\
-151.9
\end{tabular} & $\begin{array}{ll}{ }^{10} \text { B: } 6.8 & { }^{11} B: 5.9^{[\mathrm{c}]} \\
{ }^{10} \text { B: } 5.5 & { }^{11} \text { B: } 5.2^{[\mathrm{c}]} \\
{ }^{10} \text { B: } 5.4 & { }^{11} \text { B: } 4.9^{[\mathrm{c}]} \\
{ }^{10} \text { B: } 6.2 & { }^{11} \text { B: } 5.5^{[\mathrm{c}]} \\
{ }^{10} \text { B: } 2.9 & { }^{11} \text { B: } 2.8^{[\mathrm{d}]}\end{array}$ \\
\hline $\mathrm{PF}_{6}{ }^{-}$ & $\begin{array}{l}\text { dmso-d6 } \\
\text { Acetone-d6 } \\
\text { MeOH-d } 4\end{array}$ & $\begin{array}{l}-70.0 \\
-72.6 \\
-74.7\end{array}$ & $\begin{array}{l}3.8^{[\mathrm{e}]} \\
3.4^{[\mathrm{e}]} \\
3.1^{[\mathrm{e}]}\end{array}$ \\
\hline
\end{tabular}




\begin{tabular}{|l|l|l|l|}
\hline & $\mathrm{MeCN} \mathrm{d}_{3}$ & -72.8 & $3.5^{[\mathrm{e}]}$ \\
$\mathrm{CDCl}_{3}$ & -72.3 & $2.0^{[\mathrm{f}]}$ \\
\hline
\end{tabular}

[a]: taken from Aldrich, TraceCERT ${ }^{\circledR}$ CRMs for quantitative NMR. [b]: this work. [c]: using SIPr.HBF 4 . [d]:

using $\mathrm{N}(\mathrm{Pr})_{4}, \mathrm{BF}_{4}$. [e]: using SIPr.HPF 6 . [f]: using $\mathrm{N}(\mathrm{Bu})_{4}, \mathrm{PF}_{6}$.

\subsection{Ligands.}

As illustration of this methodology due to their actual increasing interest in catalysis, we decided to focus

on imidazolium and imidazolinium that are used for $\mathrm{N}$-heterocyclic carbenes (NHCs) and metal-NHCs synthesis.[4] In his seminal communications, Arduengo reports a convenient synthesis of these azoliums that consists of refluxing diamines in triethyl orthoformate (Scheme 1).[4b] After filtration, salt/triethyl orthoformate adduct are obtained and simple purification can be achieved by repeated recrystallizations from acetonitrile/ether.
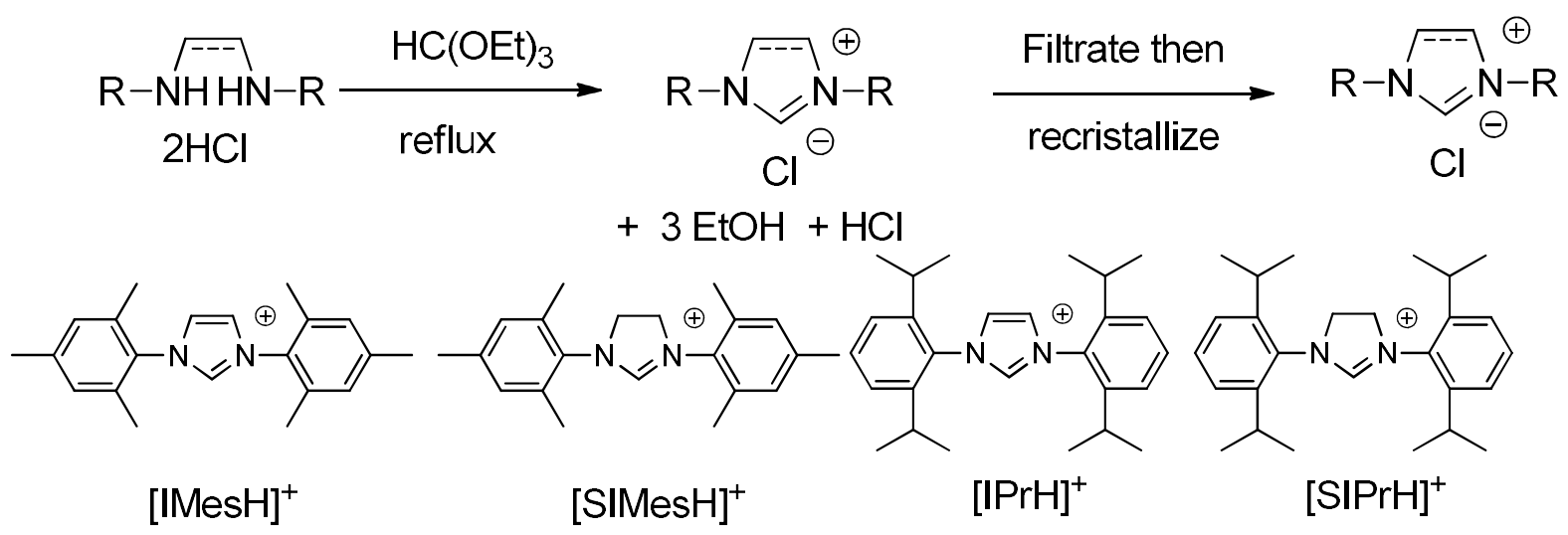

Scheme 1: Imidazolium and imidazolinium synthesis.

At first, we followed the Arduengo's procedure for SIMes. $\mathrm{HCl}$ and find, using 1,4-DMB, a purity of $72 \%$ for the crude product after filtration (Table 2 , entry 1 ). Simple recrystallization [4b] and determination of the 
water content by Karl-Fisher analysis ( 0.5 molecule of water, Table 2, entry 2 ) affords a purity of $91 \%$. For the azolium series, the amount of water varies from 0 to 1 molecule. This first screening of classical imidazolium and imidazolinium highlights the importance of the purity determination that should be corroborated with Karl-Fisher analysis results.

Table 2: purity of azolium chlorides.

\begin{tabular}{|c|c|c|}
\hline Entries $^{(a)}$ & Azolium & Purity $^{(b)}$ \\
\hline 1 & SIMes.HCl(c) & $72 \%$ \\
\hline 2 & SIMes.HCl, $0.5 \mathrm{H}_{2} \mathrm{O}^{(\mathrm{d})}$ & $91 \%( \pm 2 \%)$ \\
\hline 3 & IMes.HCI, $1 \mathrm{H}_{2} \mathrm{O}$ & $98 \%( \pm 1 \%)$ \\
\hline 4 & SIPr.HCl, $\mathbf{O H}_{2} \mathrm{O}$ & $91 \%( \pm 2 \%)$ \\
\hline 5 & IPr.HCl, $0.5 \mathrm{H}_{2} \mathrm{O}$ & $96 \%( \pm 2 \%)$ \\
\hline
\end{tabular}

(a): in dmso-d6. (b): uncertainty from 3 experiments. (c): before recrystallization. (d): after recrystallization.

The same procedure was followed for the non-coordinating anions $\mathrm{PF}_{6}$ and $\mathrm{BF}_{4}$, commonly used for metal carbene complexes. Importantly, both ${ }^{1} \mathrm{H}$ qNMR and ${ }^{19} \mathrm{~F}$ qNMR should give comparable results. We simply synthesize the $\mathrm{PF}_{6}$ and $\mathrm{BF}_{4}$ salts by anion metathesis from the corresponding azolium chloride as previously described (Table 3). [5] We obtained high yields and in contrast to the chloride series, Karl-Fisher analysis demonstrates the absence of residual water (less than $0.1 \%$ ). The results show a good correspondence between both technics with a maximum deviation of $3 \%$ (entries 6 ) and an averaged deviation of $1.8 \%$. In all cases, good purity was observed. 
Table3: Purity of azolium with non-coordinating salts.

\begin{tabular}{|c|c|c|c|c|}
\hline Entry $^{(a)}$ & Azolium & Yield & Purity by ${ }^{1} H$ qNMR ${ }^{(b)}$ & Purity by ${ }^{19} \mathrm{~F} q \mathrm{qMR}^{(\mathrm{b})}$ \\
\hline 1 & SIMes.HPF $_{6}$ & $90 \%$ & $98 \%( \pm 2 \%)$ & $97 \%( \pm 1 \%)$ \\
\hline 2 & IMes.HPF $_{6}$ & $91 \%$ & $99 \%( \pm 3 \%)$ & $99 \%( \pm 1 \%)$ \\
\hline 3 & SIPr.HPF $_{6}$ & $76 \%$ & $96 \%( \pm 2 \%)$ & $97 \%( \pm 2 \%)$ \\
\hline 4 & IPr.HPF $_{6}$ & $86 \%$ & $95 \%( \pm 2 \%)$ & $96 \%( \pm 1 \%)$ \\
\hline 5 & SIMes.HBF $_{4}$ & $89 \%$ & $98 \%( \pm 2 \%)$ & $99 \%( \pm 1 \%)$ \\
\hline 6 & IMes. $\mathrm{HBF}_{4}$ & $90 \%$ & $94 \%( \pm 2 \%)$ & $97 \%( \pm 1 \%)$ \\
\hline 7 & SIPr. $\mathrm{HBF}_{4}$ & $78 \%$ & $95 \%( \pm 2 \%)$ & $97 \%( \pm 1 \%)$ \\
\hline 8 & 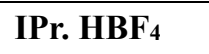 & $91 \%$ & $93 \%( \pm 2 \%)$ & $95 \%( \pm 1 \%)$ \\
\hline
\end{tabular}

(a): in dmso-d $d_{6}$ (b) Uncertainty from 2 experiments.

\subsection{Metal-NHCs.}

The purity of a selection of useful metal-NHCs was determined. We used well-established literature procedures to synthetize silver, copper, gold and palladium complexes. Indeed, $\mathrm{Ag}(\mathrm{I})-\mathrm{NHCs}$ and $\mathrm{Cu}(\mathrm{I})-$

NHCs are important synthons for transmetallation. [6] Silver(I)-NHCs is used in A3 coupling reactions and oxazole synthesis, copper(I)-NHCs are used in CuAAC, reduction, conjugated additions, allylic substitutions, alkene and alkyne transformations, nitrene transfert, allene synthesis, hydrocarboxylation, A3 coupling, oxidative coupling of phenol. [7] Regarding gold(I)-NHCs, they are used in a wide range of catalysis including enyne cycloisomerization, alkyne hydration, C-C coupling reactions; [8] and palladium(II)-NHCs has gained high importance in homogeneous cross-coupling. [9]

IMesAgCl, SIMesAgCl, IPrAgCl and SIPrAgCl were synthesized by the classical silver oxide route [4a], [10] and recrystallized from dichloromethane/pentane affording good purities (Table 4, entries 1-4) in $\mathrm{CDCl}_{3}$. At this stage, we noticed that a decomposition of the complex in presence of the maleic acid standard takes place after few hours; therefore $1,4-\mathrm{DMB}$ as secondary standard was selected. The copper 
complexes SIMesCuCl and IPrCuCl (entry 5-6) were obtained by a soft, direct metalation of the azolium using the ammonia protocol. [11] Both complexes are obtained with good purity, 93 and $98 \%$ respectively.

IPrAuCl was synthetized by transmetallation from IPrAgCl affording 93\% purity (entry 7). The cationic complex [IPrAu(MeCN)], $\mathrm{BF}_{4}$ was obtained from Strem chemical (95\%) and analyzed by ${ }^{1} \mathrm{H}$ and ${ }^{19} \mathrm{~F}$ qNMR to give an average purity of $95 \%$ in perfect agreement with the supplier's specifications (entry 8 ). Pd(PEPPSI)IPr was synthetized from the well-established procedure from the Organ's group.[12a] We find small amounts of indefinite materials in the crude mixture that were removed by washing with dichloromethane/pentane (1:5). The NMR spectrum shows the presence of 0.8 molecules of $\mathrm{CH}_{2} \mathrm{Cl}_{2}$ that cannot be removed under reduced pressure (2mbar) for several hours. [12b] The Pd(PEPPSI)IPr's purity in this mixture is found to be $87 \%$ and, taking the dichloromethane amount into account, a $96 \%$ purity is obtained (entry 9).

Table 4: Purity of metal-NHCs.

\begin{tabular}{|c|c|c|c|}
\hline Entries & M-NHC & Solvent & Purity \\
\hline 1 & IMesAgCI & $\mathrm{CDCl}_{3}$ & $97 \%( \pm 1 \%)$ \\
\hline 2 & SIMesAgCI & $\mathrm{CDCl}_{3}$ & $95 \%( \pm 2 \%)$ \\
\hline 3 & IPrAgCl & $\mathrm{CDCl}_{3}$ & $97 \%( \pm 1 \%)$ \\
\hline 4 & SIPrAgCl & $\mathrm{CDCl}_{3}$ & $98 \%( \pm 1 \%)$ \\
\hline 5 & SIMesCuCl & $\mathrm{CDCl}_{3}$ & $93 \%( \pm 2 \%)$ \\
\hline 6 & IPrCuCl & $\mathrm{CDCl}_{3}$ & $98 \%( \pm 1 \%)$ \\
\hline 7 & IPrAuCl & dmso-d6 & $93 \%( \pm 1 \%)$ \\
\hline 8 & [IPrAu,MeCN], $\mathrm{BF}_{4}$ & dmso-d 6 & $\begin{array}{l}94 \%( \pm 1 \%)^{(b)} \\
96 \%( \pm 1 \%)^{(c)}\end{array}$ \\
\hline 9 & $\mathrm{Pd}(\mathrm{PEPPSI}) \mathrm{IPr}^{\left({ }^{(\mathrm{d})}\right)} \mathrm{0.8C}_{2} \mathrm{Cl}_{2}$ & $\mathrm{CDCl}_{3}$ & $96 \%( \pm 2 \%)$ \\
\hline
\end{tabular}

(a): average of two measurements. (b): by ${ }^{1} H$ qNMR. (c): by ${ }^{19} F$ qNMR. (d): Pd(PEPPSI)IPr: Dichloro[1,3bis(2,6-diisopropylphenyl)imidazol-2-ylidene)](3-chloropyridyl)palladium(II). 


\section{Conclusion}

In conclusion, we have applied ${ }^{1} \mathrm{H}$ and ${ }^{19} \mathrm{~F}$ quantitative NMR to organometallics complexes and their corresponding ligands. According to previous reports, this technique is easy to conduct, affording purity of compounds in approximately 30 minutes to $1 \mathrm{~h}$ experiment. Care should be taken regarding the choice of the solvent and if possible, Karl Fisher analysis should be considered, especially regarding the ligands.

\section{Acknowledgment}

Financial support from the Centre National de la Recherche Scientifique (CNRS) and the Université Clermont-Auvergne are acknowledged.

\section{Appendix.}

Supplementary material: procedure, NMR sequence and spectra can be found, in the online version, at ...

\section{References.}

[1] (a) G.F. Pauli, S. N. Chen, C. Simmler, D. C. Lankin, T. Gödecke, B.U. Jaki, J. B. Friesen, J. B. McAlpine, J. G. Napolitano, Importance of purity evaluation and the potential of quantitative ${ }^{1} \mathrm{H}$ NMR as a purity assay. J. Med. Chem. 57 (2014) 9220-31. https://doi.org/10.1021/jm500734a. (b) S.K. Bharti, R. Roy, Quantitative 
${ }^{1} \mathrm{H} \quad$ NMR spectroscopy Trends in analytical Chemistry $35 \quad$ (2012) 5-26. https://doi.org/10.1016/j.trac.2012.02.007.

[2] M. Cushman, G. I. Georg, U. Holzgrabe, S. Wange, Absolute Quantitative ${ }^{1} \mathrm{H}$ NMR Spectroscopy for Compound Purity Determination. J. Med. Chem. 57 (2014) 9219. https://doi.org/10.1021/jm501683w.

[3] Purification of laboratory chemicals 6thEds, p279.

[4] (a) M. N. Hopkinson, C. Richter, M. Schedler, F. Glorius, An overview of N-heterocyclic carbenes. Nature 510, (2014), 485-496. https://doi.org/10.1038/nature13384. (b) A. J. Arduengo, R. Krafczyk, R. Schmutzler, H. A. Craig, J. R. Goerlich, W. J. Marshall, M. Unverzagt, Imidazolylidenes, imidazolinylidenes and imidazolidines, Tetrahedron 55 (1999) 14523-34. https://doi.org/10.1016/S0040-4020(99)00927-8.

[5] X. Bantreil, S. P. Nolan, Synthesis of N-heterocyclic carbene ligands and derived ruthenium olefin metathesis catalysts. Nat. Protocols 6 (2011) 69-77. https://doi.org/10.1038/nprot.2010.177.

[6] (a) I. J. B Lin, C. S. Vasam, Preparation and application of N-Heterocyclic carbene complexes of $\mathrm{Ag}(\mathrm{I})$ Coord. Chem. Rev. 251 (2007) 642-670. https://doi.org/10.1016/j.ccr.2006.09.004. (b) F. Nahra, A. GómezHerrera, C. S. J. Cazin, Copper(I)-NHC complexes as NHC transfer agents. Dalton Trans. 46 (2017) 628-631. https://doi.org/10.1039/C6DT03687B.

[7] (a) V. A. Peshkov, O. P. Pereshivko, E. V. Van der Eycken, A walk around the A3-coupling. Chem. Soc. Rev. 41 (2012) 3790-3807. https://doi.org/10.1039/C2CS15356D. (b) R. Heath, H. Müller-Bunz, M. 
Albrecht, Silver(I) NHC mediated C-C bond activation of alkyl nitriles and catalytic efficiency in oxazoline synthesis. Chem. Commun. 51 (2015) 8699-8701. https://doi.org/10.1039/C5CC02558C.

[8] S. P. Nolan, The Development and Catalytic Uses of N-Heterocyclic Carbene Gold Complexes. Acc. Chem. Res. 44 (2011) 91-100. https://doi.org/10.1021/ar1000764.

[9] G. C. Fortman, S. P. Nolan N-Heterocyclic carbene (NHC) ligands and palladium in homogeneous crosscoupling catalysis: a perfect union. Chem. Soc. Rev. $40 \quad$ (2011) 5151-5169. https://doi.org/10.1039/C1CS15088J.

[10] P. de Frémont, N. M. Scott, E. D. Stevens, T. Ramnial, O. C. Lightbody, C. L. B. Mac Donald, J. A. C. Clyburne, C. D. Abernethy, S. P. Nolan Synthesis of Well-Defined N-Heterocyclic Carbene Silver(I) Complexes. Organometallics 22 (2005) 6301-6309. https://doi.org/10.1021/om050735i.

[11] C. Gibard, H. Ibrahim, A. Gautier, F. Cisnetti, Simplified Preparation of Copper(I) NHCs Using Aqueous Ammonia. Organometallics 15 (2013) 4279-4283. https://doi.org/10.1021/om400440b.

[12] (a) J. Nasielski, N. Hadei, G. Achonduh, E. Ass en, B. Kantchev, C. J. O'Brien, A. Lough, M. G. Organ. Structure-Activity Relationship Analysis of Pd-PEPPSI Complexes in Cross-Couplings: A Close Inspection of the Catalytic Cycle and the Precatalyst Activation Mode. Chem. Eur. J. 16 (2010) 10844-53. https://doi.org/10.1002/chem.201000138. (b) We do not try to increase the temperature. 


\section{Application of Quantitative ${ }^{1} \mathrm{H}$ and ${ }^{19} \mathrm{~F}$ NMR to Organometallics.}

Ayman Akhdar, ${ }^{\mathrm{a}}$ Jean-Michel Andanson, ${ }^{\mathrm{a}}$ Sophie Faure, ${ }^{\mathrm{a}}$ Mounir Traïkia, ${ }^{\mathrm{a}}$ and Arnaud Gautier ${ }^{\mathrm{*}}$

S1. Generals. 14

S2. Structures 15

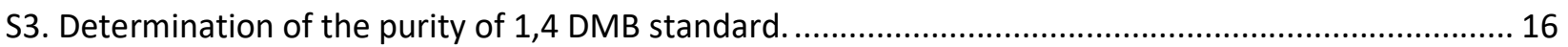

S4. ${ }^{19} \mathrm{~F}$ NMR spectra of SIPr.HPF

S5. ${ }^{19} \mathrm{~F}$ NMR spectra of SIPr.HBF

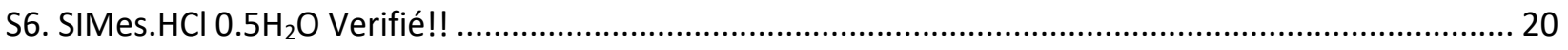

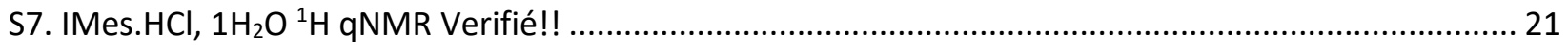

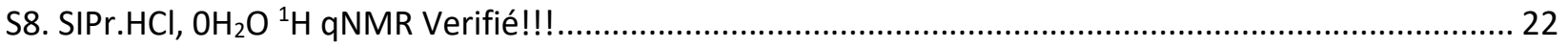

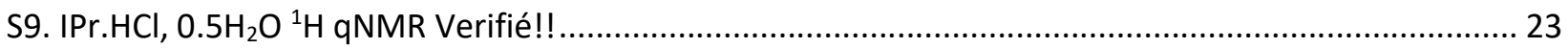

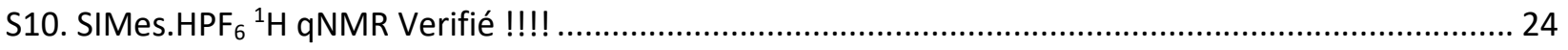

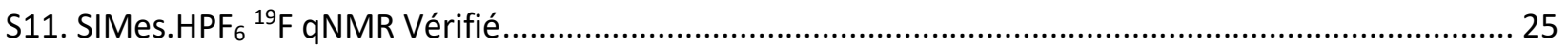

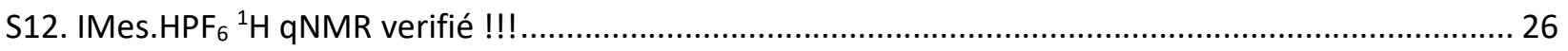

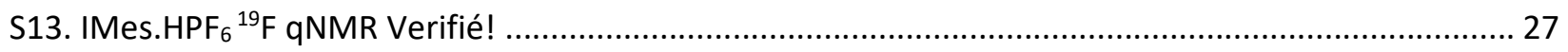

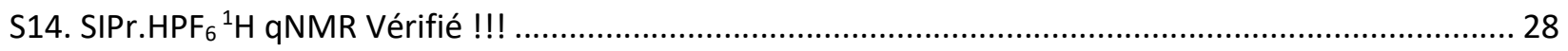

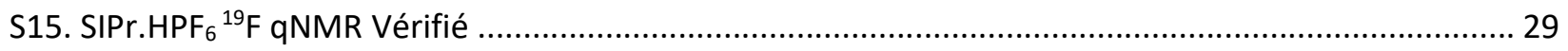

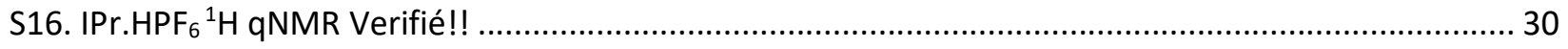

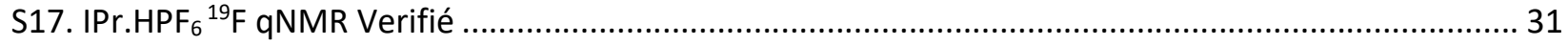

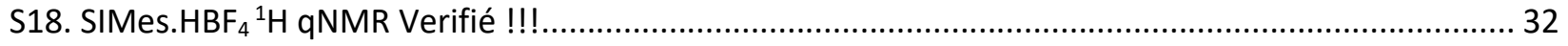

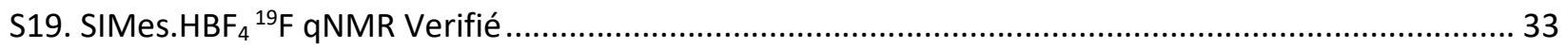

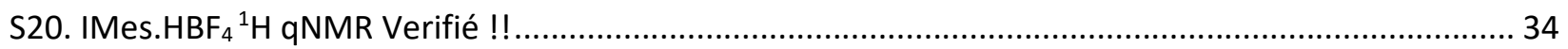

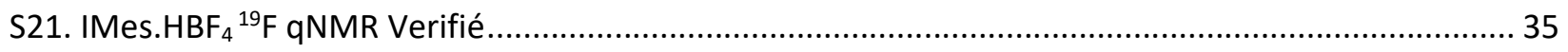

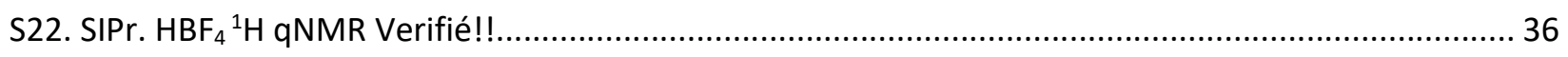

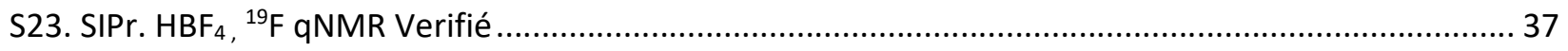




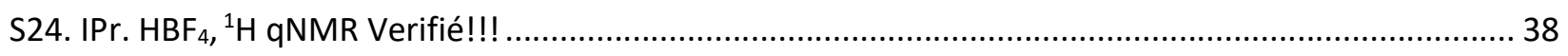

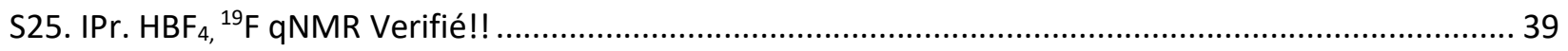

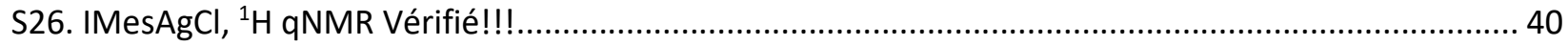

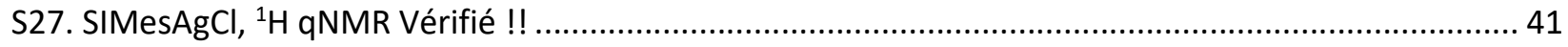

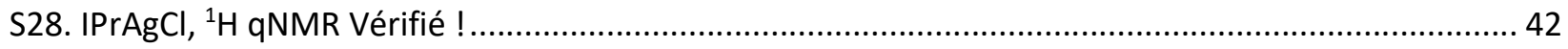

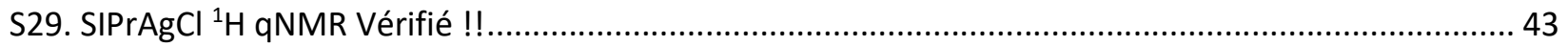

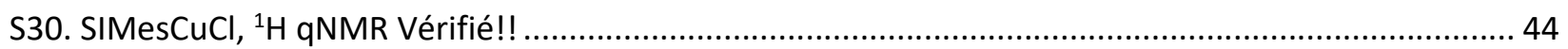

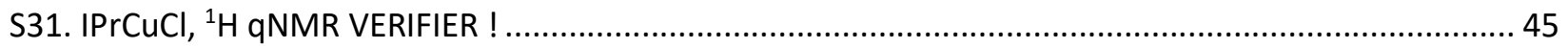

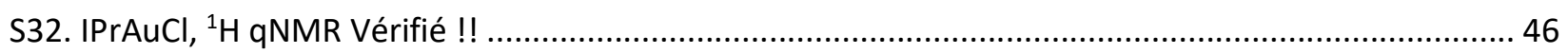

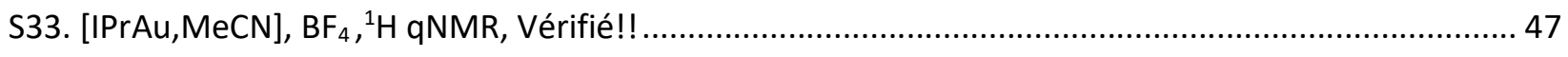

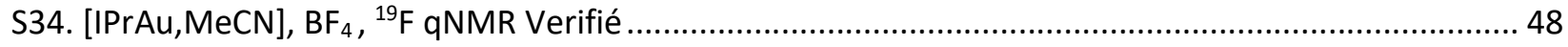

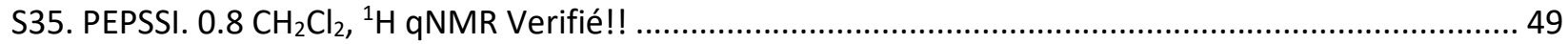




\section{S1. Generals.}

All ligands and complexes were analyzed by ${ }^{1} \mathrm{H}$ and $\{1 \mathrm{H}\}^{19} \mathrm{~F}$ NMR spectroscopy on a Bruker advance 400 spectrometer. $\mathrm{CDCl}_{3}$ was neutralized over anhydrous $\mathrm{K}_{2} \mathrm{CO}_{3}$ prior to use. The water content was measured using a coulometric Karl Fischer titrator (MettlerToledo, DL32).

Sample Preparation: The sample and the standard are weighed using a Mettler Toledo balance $(0.01 \mathrm{mg}$ accuracy) into a single Eppendorf, then solvent was added and the recipient was gently shaken to ensure a total dissolution. The solution was transferred into a $3 \mathrm{~mm}$ standard NMR tube for analysis.

Pulse Program: Sample Temperature: $19^{\circ} \mathrm{C}$ (regulated $\pm 0.1 \mathrm{~K}$ ), single pulse, without carbon decoupling ('zg' with $90^{\circ}$ pulse). Data Points (acquired): $64 \mathrm{~K} . \mathrm{NS}=64$. Relaxation delay: D1=60s. Acquisition Time: 4s. Spectral window for proton: $\mathrm{SW}=30 \mathrm{ppm}$ and $01: 7.5 \mathrm{ppm}$; for fluorine, $\mathrm{PF} 6 \mathrm{SW}=120 \mathrm{ppm}$ and $\mathrm{O}_{1}=90$ ppm, $\mathrm{BF}_{4}: \mathrm{SW}=-130 \mathrm{ppm}$ and $\mathrm{O} 1=100 \mathrm{ppm}$.

Post-Acquisition Processing was performed with ACDLABS software (1.2, academic version). Zero Filling: to $256 \mathrm{~K}$. Line Broadening: $\mathrm{LB}=0.1 \mathrm{~Hz}$. Phasing: manually. Baseline Correction, for ${ }^{1} \mathrm{H} N \mathrm{NR}$ : $6^{\text {th }}$ order polynomial. For each signal measured a ratio signal/noise $>100$ is verified. 


\section{S2. Structures}

\section{Ligands}
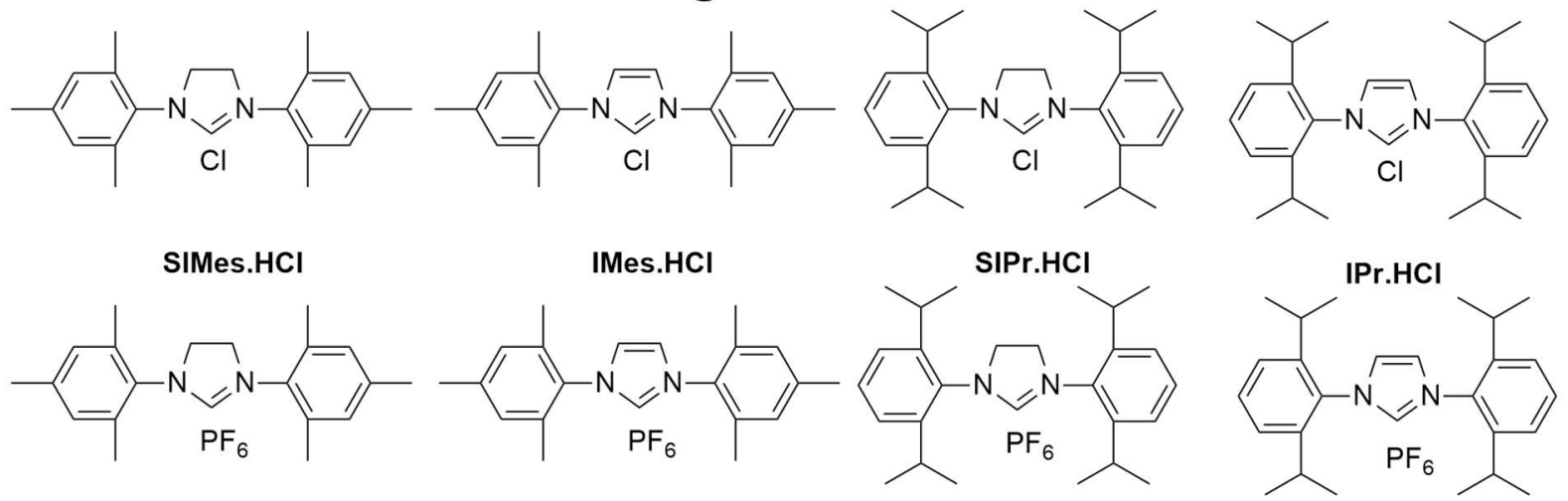

SIMes.HPF 6

IMes.HPF $_{6}$

SIPr.HPF 6

IPr.HPF $_{6}$
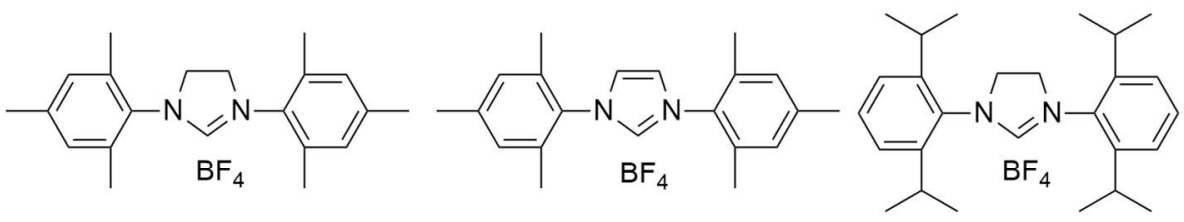

SIMes.HBF 4

IMes.HBF 4

SIPr.HBF 4

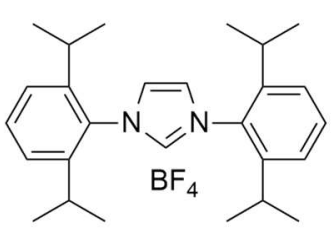

\section{Complexes}

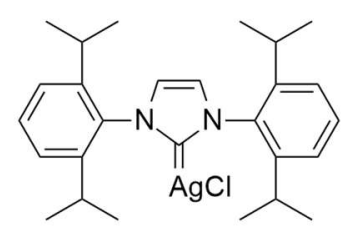

IPrAgCI<smiles>CC(C)c1cccc(C(C)C)c1-n1ccn(-c2c(C(C)C)cccc2C(C)C)c1=[Cr]Cl</smiles>

IPrCuCl

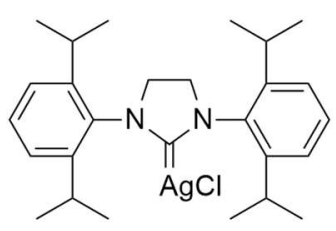

SIPrAgCI<smiles>CC(C)c1cccc(C(C)C)c1N1C=CN(c2c(C(C)C)cccc2C(C)C)C1=[Ge]Cl</smiles>

IPrAuCI

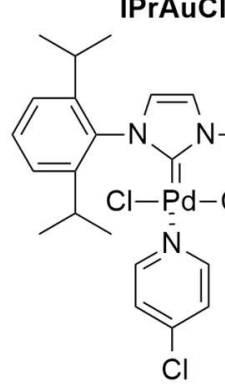

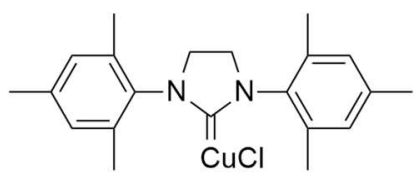

SIMesCuCl

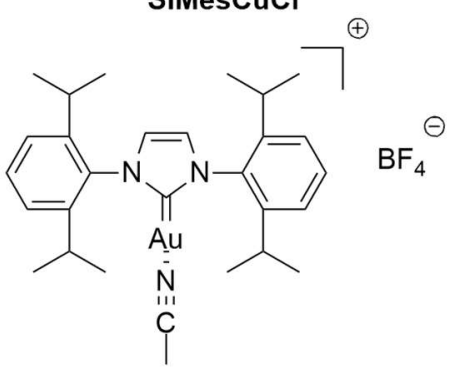

$[\mathrm{IPrAu}(\mathrm{MeCN})]^{+}, \mathrm{Cl}^{-}$

PEPPSI 


\section{S3. Determination of the purity of 1,4 DMB standard.}

Purity was determined as the average of 6 independent measurements in dmso_ $d_{6}$ from maleic acid gold standard trace certified (Aldrich) as primary standard.

Example of spectrum

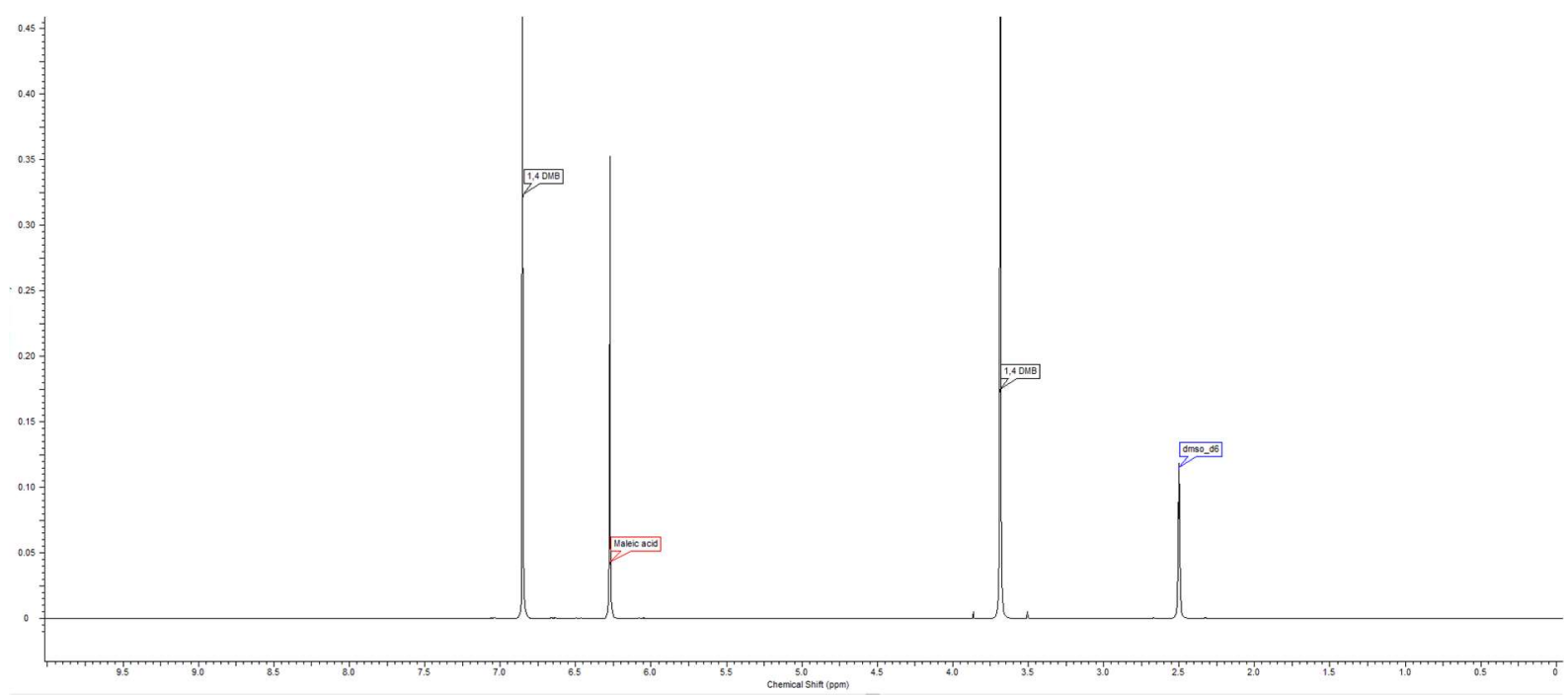

Purity is calculated as the ratio of the number of mol of 1,4-DMB determined by qNMR divided by the number of mol of 1,4-DMB weighted, taking into account of the primary standard purity $(99.98+/-0.13$ $\%)$.

\begin{tabular}{|c|c|c|c|c|c|c|}
\hline Exp & 1 & 2 & 3 & 4 & 5 & 6 \\
\hline Purity & 99.32 & 98.39 & 98.91 & 99.56 & 100.00 & 100.04 \\
\hline Average & $99.48 \%$ & & & & & \\
\hline $\begin{array}{l}\text { Standard } \\
\text { deviation }\end{array}$ & $+/-0.06 \%$ & & & & & \\
\hline
\end{tabular}

The linearity of the response in the range 10 to $25 \mathrm{mg}$ (0.08 to $0.17 \mathrm{mmol})$ was verified: 


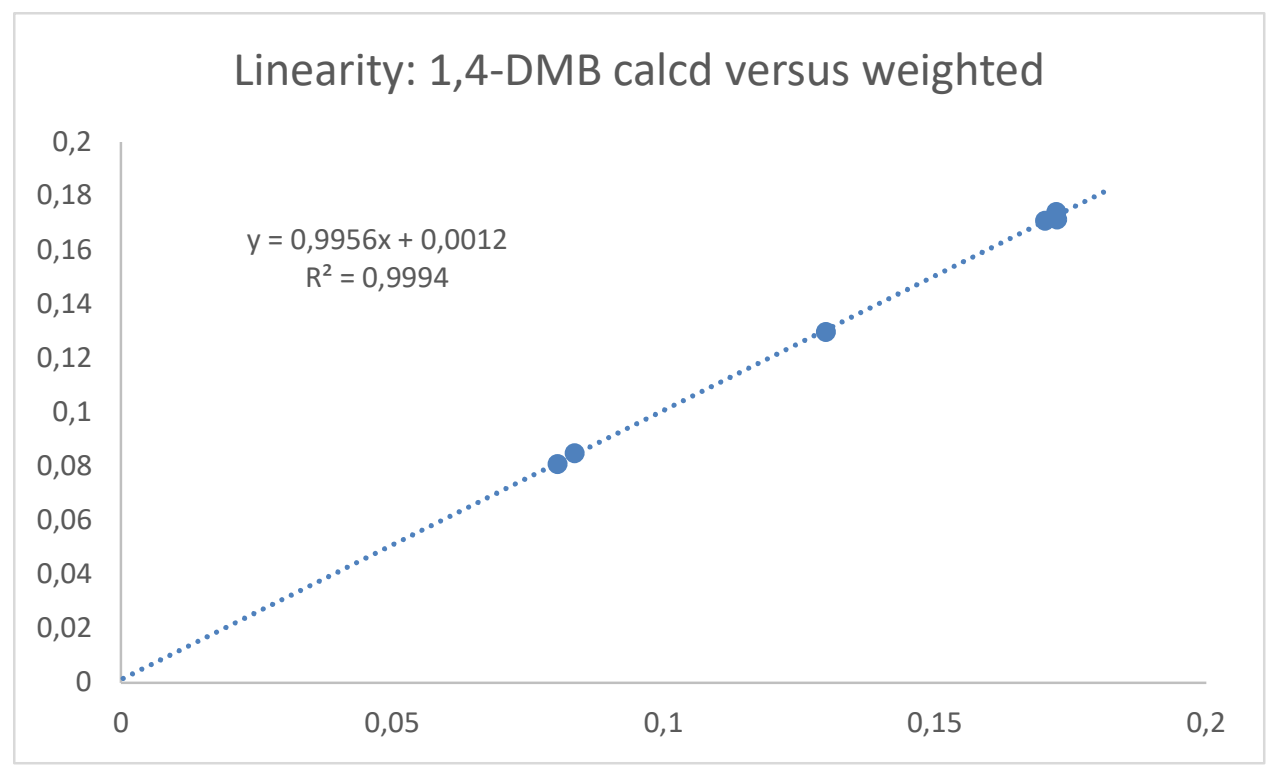


S4. ${ }^{19}$ F NMR spectra of SIPr.HPF 6

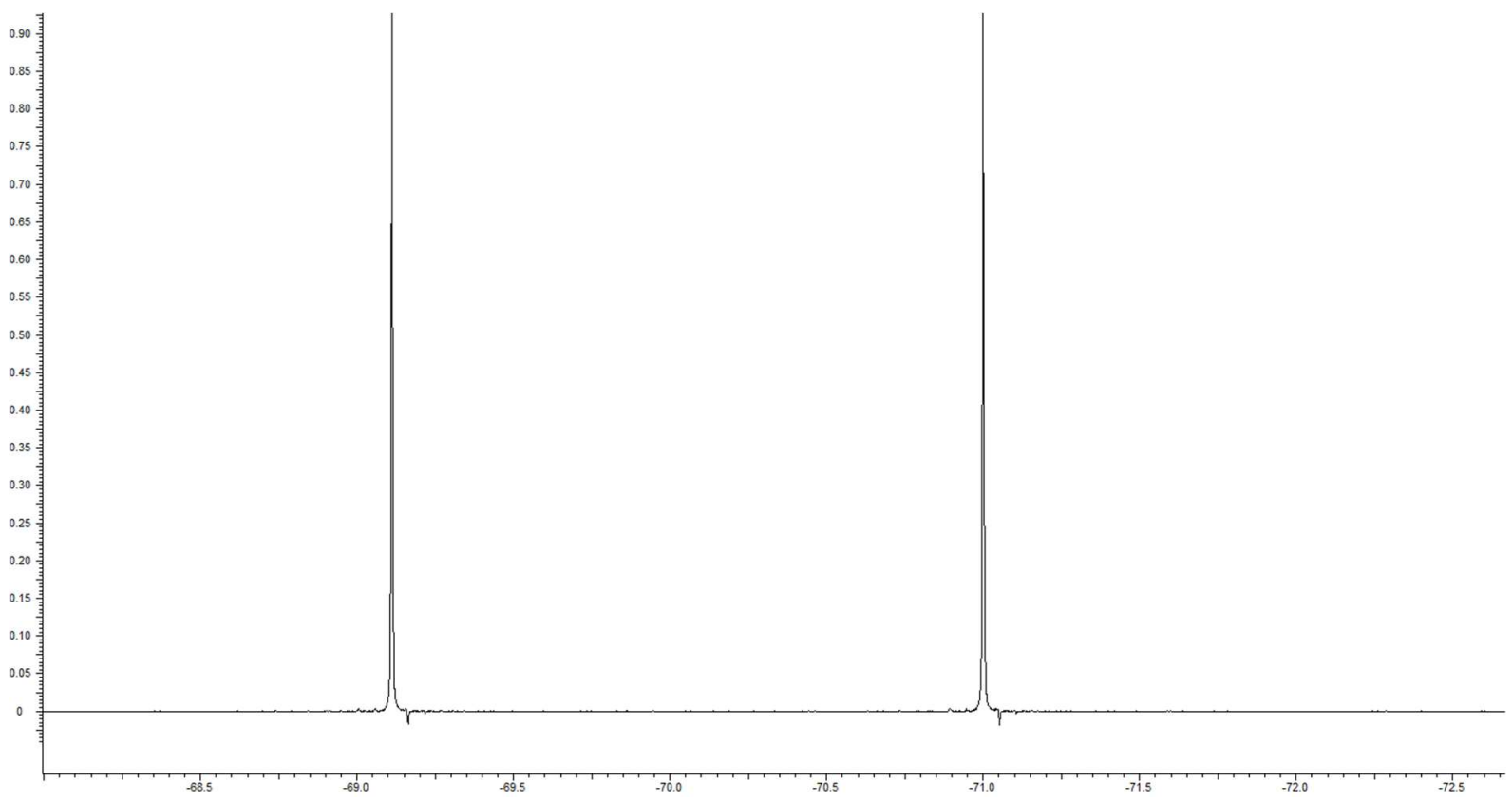


S5. ${ }^{19}$ F NMR spectra of SIPr.HBF 4

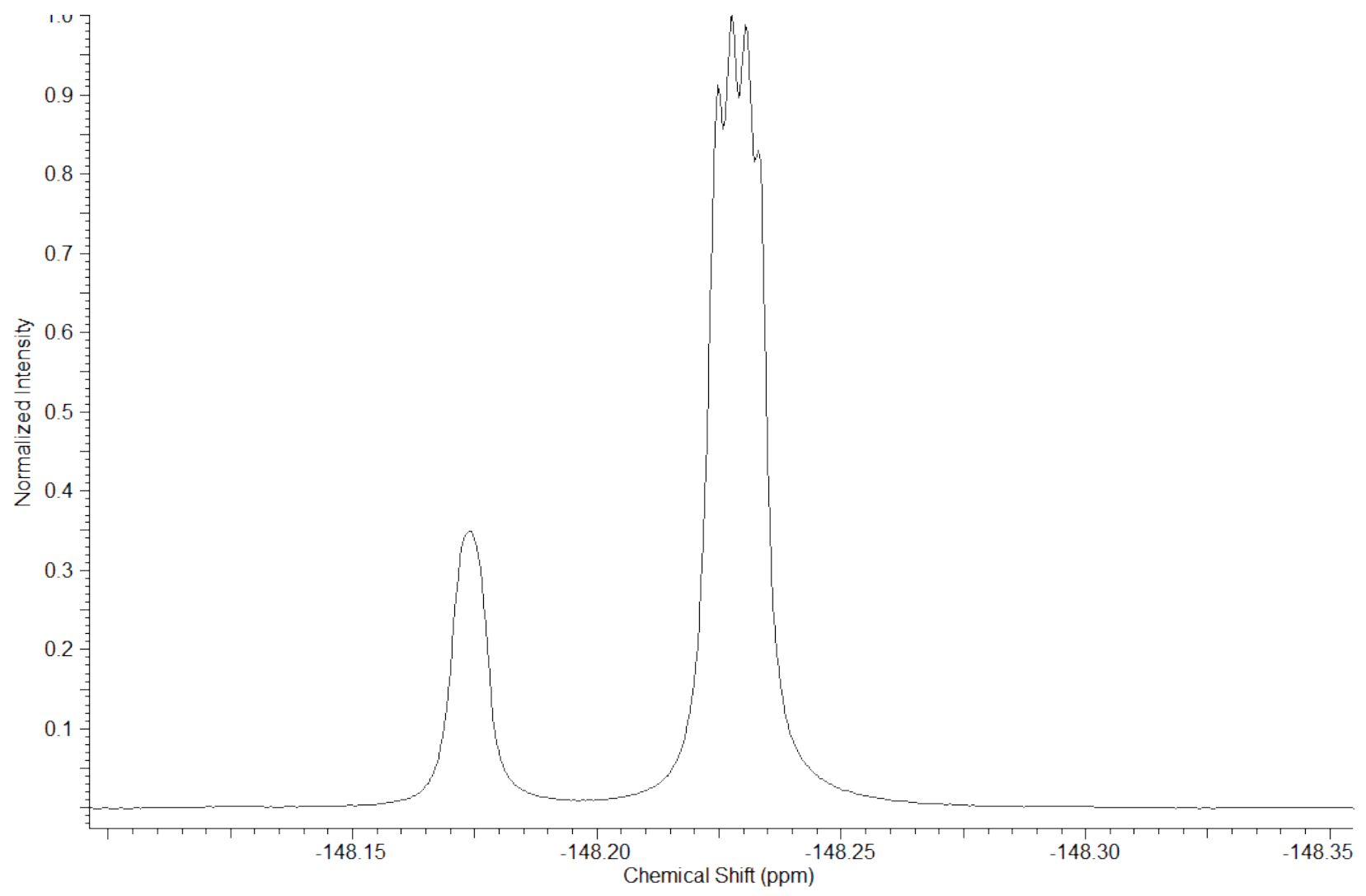




\section{S6. SIMes.HCl $0.5 \mathrm{H}_{2} \mathrm{O}$ Verifiée!!}

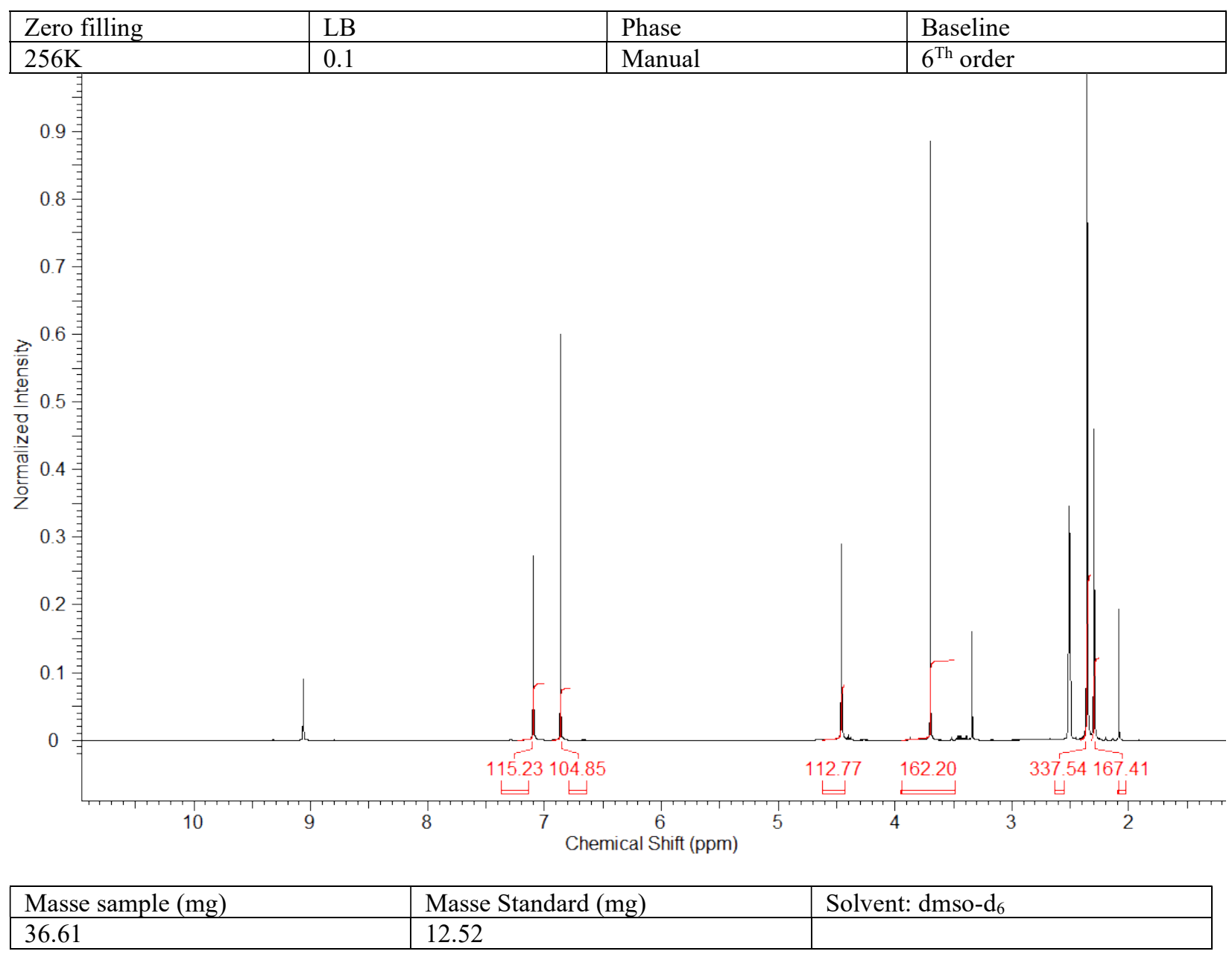

\begin{tabular}{|l|l|l|l|l|}
\hline Signals standard $(\mathrm{ppm})$ & 6.86 & 3.7 & & \\
\hline Integration & 104.8 & 162.2 & & \\
\hline \hline Signals sample $(\mathrm{ppm})$ & 7.1 & 4.45 & 2.35 & 2.3 \\
\hline Integration & 115.2 & 112.7 & 337.5 & 167.4 \\
\hline
\end{tabular}

Somme of the integral: sample, 732.8; standard, 267.

Number of protons: sample, 26; standard, 10.

Molecular weight: sample, 351.9; standard, 138.2.

Mass of: sample, $36.61 ;$ standard: 12.52.

$P_{s}=\frac{I_{s}}{I_{s t d}} \cdot \frac{N_{\text {std }}}{N_{s}} \cdot \frac{M_{s}}{M_{s t d}} \cdot \frac{m_{\text {std }}}{m_{s}} \cdot P_{s t d}=\frac{732.8}{267} \cdot \frac{10}{26} \cdot \frac{351.9}{138.2} \cdot \frac{12.52}{36.61} \cdot 0.995=0.914$

\section{Purity: $91 \%$}




\section{S7. IMes.HCl, $\mathbf{1 H}_{2} \mathrm{O}^{1} \mathrm{H}$ qNMR Verifié!!}

\begin{tabular}{|l|l|l|l|}
\hline Zero filling & LB & Phase & Baseline \\
\hline $256 \mathrm{~K}$ & 0.1 & Manual & $6^{\mathrm{Th}}$ order \\
\hline
\end{tabular}

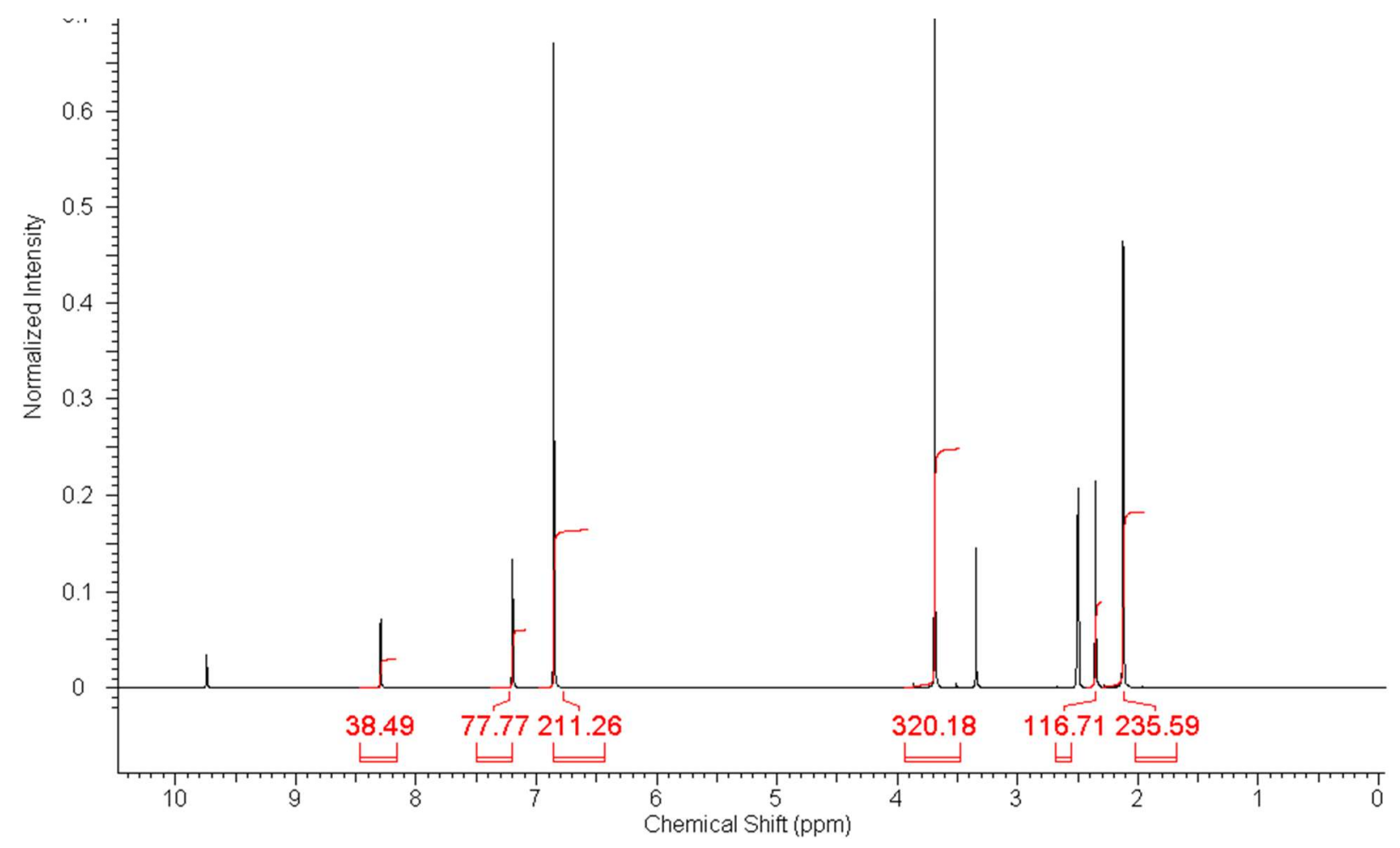

\begin{tabular}{|l|l|l|}
\hline Masse sample $(\mathrm{mg})$ & Masse Standard $(\mathrm{mg})$ & Solvent: $\mathrm{dmso}_{\mathrm{N}} \mathrm{d}_{6}$ \\
\hline 13.48 & 13.96 & \\
\hline
\end{tabular}

\begin{tabular}{|l|l|l|l|l|}
\hline Signals standard (ppm) & 6.86 & 3.7 & & \\
\hline Integration & 211.3 & 320.2 & & \\
\hline \hline Signals sample (ppm) & 8.3 & 7.2 & 2.4 & 2.1 \\
\hline Integration & 38.5 & 77.7 & 116.7 & 235.6 \\
\hline
\end{tabular}

Somme of the integral: sample, 468.5; standard, 531.5.

Number of protons: sample, 24; standard, 10.

Molecular weight: sample, 358.9; standard, 138.2.

Mass of: sample, 13.48; standard: 13.96.

$$
P_{s}=\frac{I_{s}}{I_{s t d}} \cdot \frac{N_{s t d}}{N_{s}} \cdot \frac{M_{s}}{M_{\text {std }}} \cdot \frac{m_{\text {std }}}{m_{s}} \cdot P_{\text {std }}=\frac{468.5}{531.5} \cdot \frac{10}{24} \cdot \frac{358.9}{138.2} \cdot \frac{13.96}{13.48} \cdot 0.995=0.983
$$

\section{Purity: 98\%}


S8. SIPr.HCl, $\mathbf{O H}_{2} \mathrm{O}^{1} \mathrm{H}$ qNMR Verifié!!!

\begin{tabular}{|l|l|l|l|}
\hline Zero filling & LB & Phase & Baseline \\
\hline $256 \mathrm{~K}$ & 0.1 & Manual & $6^{\mathrm{Th}}$ order \\
\hline
\end{tabular}

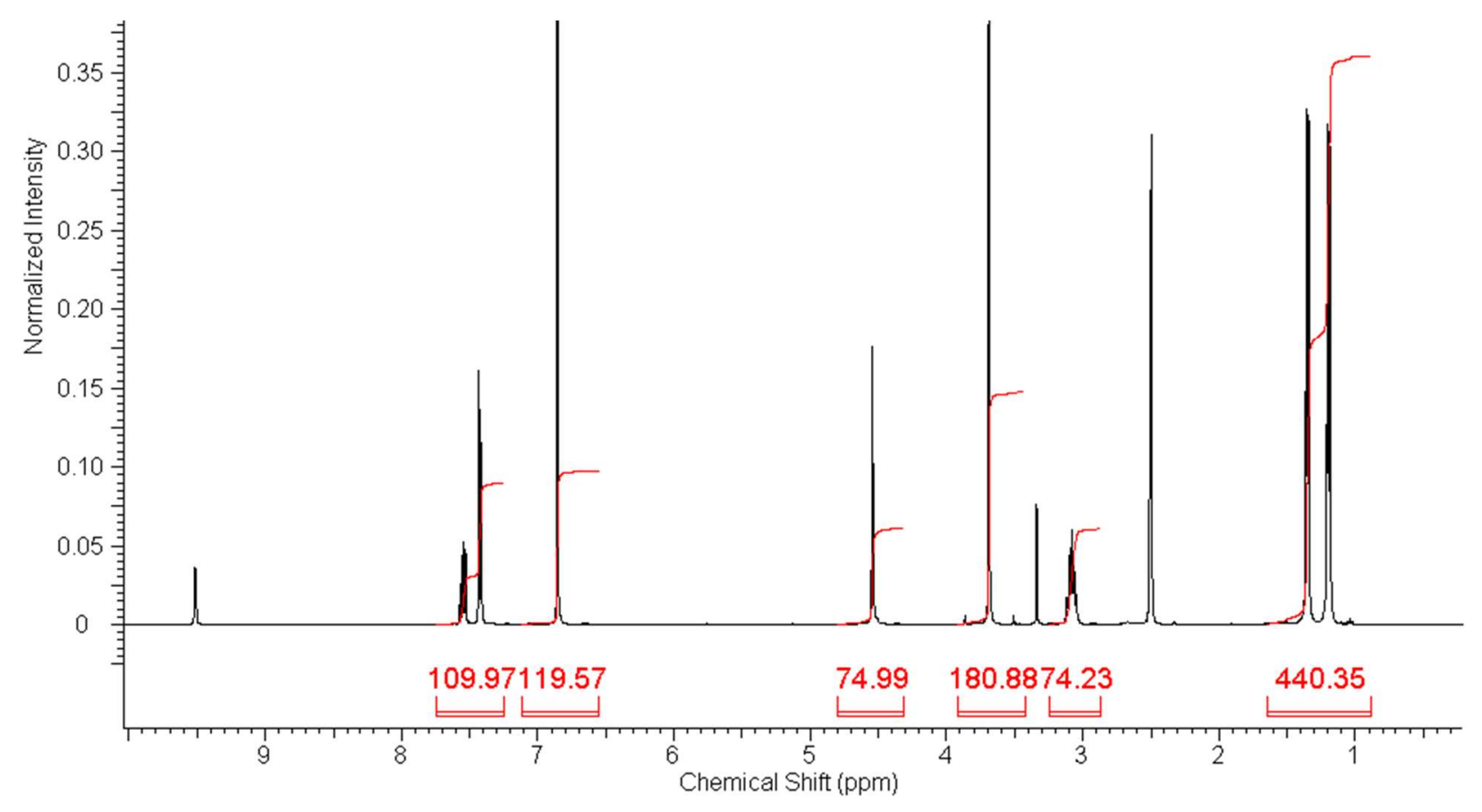

\begin{tabular}{|l|l|l|}
\hline Masse sample $(\mathrm{mg})$ & Masse Standard $(\mathrm{mg})$ & Solvent: dmso- $_{6}$ \\
\hline 31.98 & 15.53 & \\
\hline
\end{tabular}

\begin{tabular}{|l|l|l|l|l|}
\hline Signals standard $(\mathrm{ppm})$ & 6.86 & 3.7 & & \\
\hline Integration & 119.6 & 180.9 & & \\
\hline \hline Signals sample $(\mathrm{ppm})$ & 7.5 & 4.5 & 3.1 & 2.3 \\
\hline Integration & 110 & 75 & 74.2 & 440.4 \\
\hline
\end{tabular}

Somme of the integral: sample, 699.6; standard, 300.5 .

Number of protons: sample, 40; standard, 10.

Molecular weight: sample, 445.1; standard, 138.2.

Mass of: sample, 31.98; standard: 15.53.

$$
P_{s}=\frac{I_{s}}{I_{s t d}} \cdot \frac{N_{s t d}}{N_{s}} \cdot \frac{M_{s}}{M_{s t d}} \cdot \frac{m_{s t d}}{m_{s}} \cdot P_{s t d}=\frac{699.6}{300.5} \cdot \frac{10}{40} \cdot \frac{445.1}{138.2} \cdot \frac{15.53}{31.98} \cdot 0.995=0.906
$$

\section{Purity: $91 \%$}




\section{S9. IPr.HCl, $0.5 \mathrm{H}_{2} \mathrm{O}^{1} \mathrm{H}$ qNMR Verifié!!}

\begin{tabular}{|l|l|l|l|}
\hline Zero filling & LB & Phase & Baseline \\
\hline $256 \mathrm{~K}$ & 0.1 & Manual & $6^{\text {Th }}$ order \\
\hline
\end{tabular}

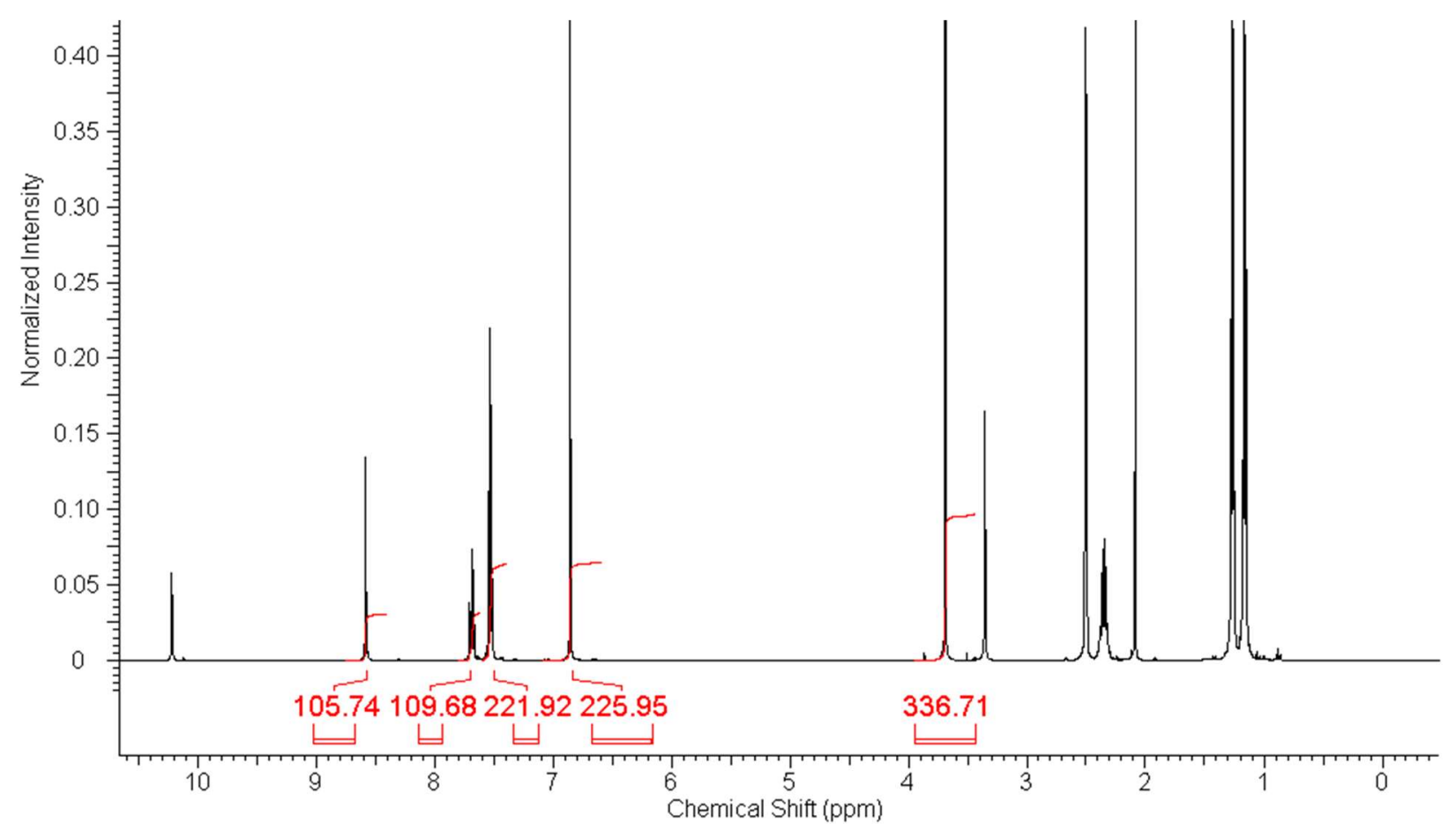

\begin{tabular}{|l|l|l|}
\hline Masse sample (mg) & Masse Standard (mg) & Solvent: dmso- $\mathrm{d}_{6}$ \\
\hline 31.75 & 11.27 & \\
\hline
\end{tabular}

\begin{tabular}{|l|l|l|l|}
\hline Signals standard (ppm) & 6.86 & 3.7 & \\
\hline Integration & 226 & 336.7 & \\
\hline \hline Signals sample (ppm) & 8.6 & 7.7 & 3.7 \\
\hline Integration & 105.7 & 109.7 & 221.9 \\
\hline
\end{tabular}

Somme of the integral: sample, 437.3; standard, 562.7.

Number of protons: sample, 8; standard, 10.

Molecular weight: sample, 434.1; standard, 138.2.

Mass of: sample, 31.75; standard: 11.27.

$$
P_{s}=\frac{I_{s}}{I_{s t d}} \cdot \frac{N_{s t d}}{N_{s}} \cdot \frac{M_{s}}{M_{s t d}} \cdot \frac{m_{s t d}}{m_{s}} \cdot P_{s t d}=\frac{552.1}{562.7} \cdot \frac{10}{8} \cdot \frac{434.1}{138.2} \cdot \frac{11.27}{31.75} \cdot 0.995=0.96
$$

\section{Purity: $96 \%$}


S10. SIMes.HPF $6{ }^{1} \mathrm{H}$ qNMR Verifié !!!!

\begin{tabular}{|l|l|l|l|}
\hline Zero filling & LB & Phase & Baseline \\
\hline $256 \mathrm{~K}$ & 0.1 & Manual & $6^{\text {Th }}$ order \\
\hline
\end{tabular}

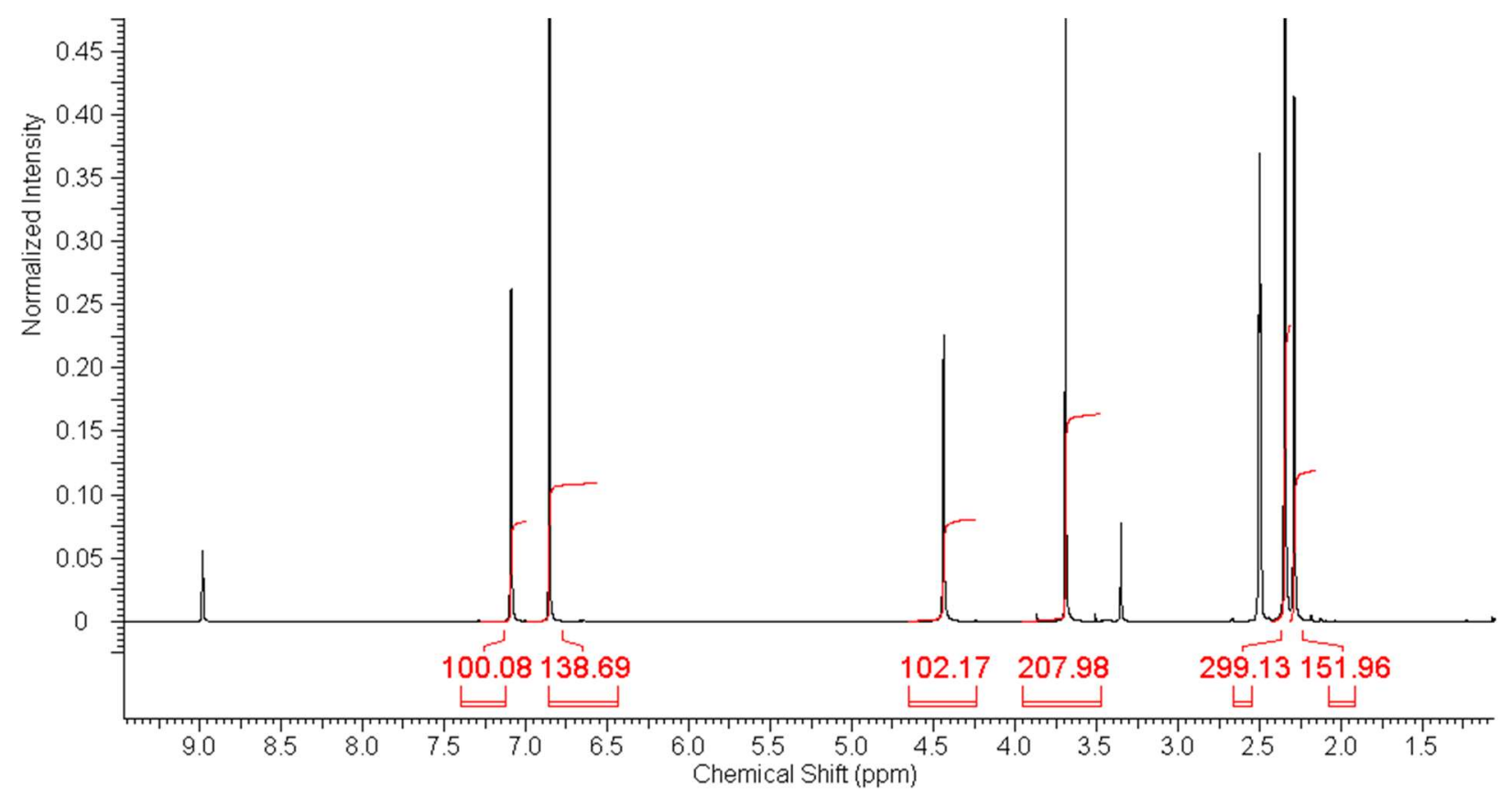

\begin{tabular}{|l|l|l|}
\hline Masse sample (mg) & Masse Standard (mg) & Solvent: dmso- $\mathrm{d}_{6}$ \\
\hline 31.00 & 12.98 & \\
\hline
\end{tabular}

\begin{tabular}{|l|l|l|l|l|}
\hline Signals standard (ppm) & 6.86 & 3.7 & & \\
\hline Integration & 138.7 & 208 & & \\
\hline \hline Signals sample (ppm) & 7.1 & 4.43 & 2.34 & 2.29 \\
\hline Integration & 100.1 & 102.7 & 299.1 & 152 \\
\hline
\end{tabular}

Somme of the integral: sample, 653.9; standard, 346.7.

Number of protons: sample, 26; standard, 10.

Molecular weight: sample, 452.4; standard, 138.2.

Mass of: sample, $30.00 ;$ standard: 12.98.

$$
P_{s}=\frac{I_{s}}{I_{s t d}} \cdot \frac{N_{s t d}}{N_{s}} \cdot \frac{M_{s}}{M_{s t d}} \cdot \frac{m_{s t d}}{m_{s}} \cdot P_{s t d}=\frac{653.9}{346.7} \cdot \frac{10}{26} \cdot \frac{452.4}{138.2} \cdot \frac{12.98}{31} \cdot 0.995=0.98
$$

\section{Purity: $98 \%$}




\section{S11. SIMes.HPF $6{ }^{19} \mathrm{~F}$ qNMR Vérifié}

\begin{tabular}{|l|l|l|l|}
\hline Zero filling & LB & Phase & Baseline \\
\hline $256 \mathrm{~K}$ & 0.1 & Manual & $6^{\text {Th }}$ order \\
\hline
\end{tabular}

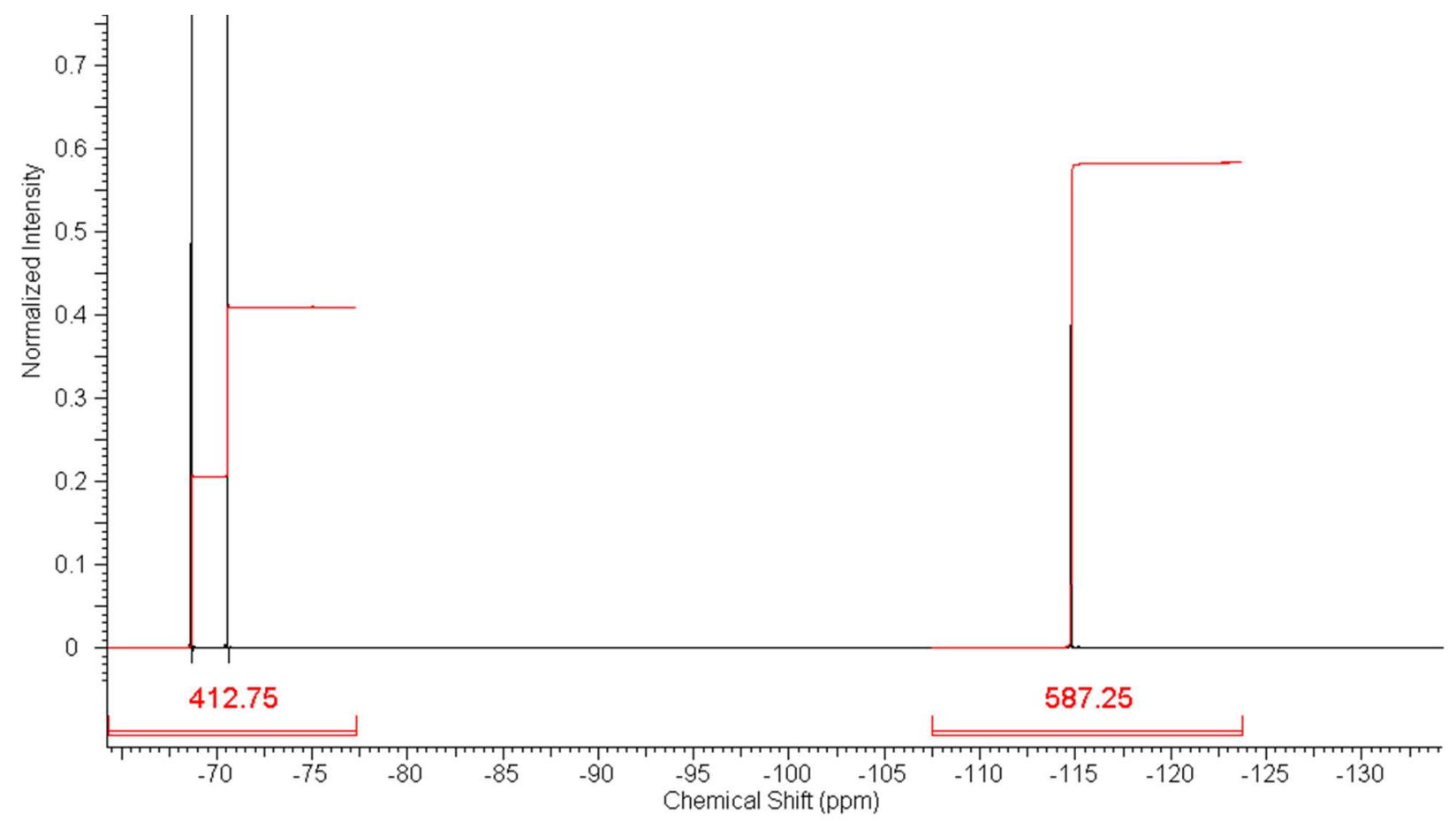

\begin{tabular}{|l|l|l|}
\hline Masse sample $(\mathrm{mg})$ & Masse Standard $(\mathrm{mg})$ & Solvent: $\mathrm{dmso}_{\mathrm{-}} \mathrm{d}_{6}$ \\
\hline 10.64 & 28.14 & \\
\hline
\end{tabular}

\begin{tabular}{|l|l|}
\hline Signals standard $(\mathrm{ppm})$ & -115 \\
\hline Integration & 587.3 \\
\hline \hline Signals sample (ppm) & -70 \\
\hline Integration & 412.8 \\
\hline
\end{tabular}

Somme of the integral: sample, 412.8; standard, 587.3.

Number of fluorine: sample, 6; standard, 1.

Molecular weight: sample, 453.4; standard, 144.6.

Mass of: sample, 10.64; standard: 28.4 .

$$
P_{s}=\frac{I_{s}}{I_{s t d}} \cdot \frac{N_{s t d}}{N_{s}} \cdot \frac{M_{s}}{M_{s t d}} \cdot \frac{m_{s t d}}{m_{s}} \cdot P_{s t d}=\frac{412.8}{587.3} \cdot \frac{1}{6} \cdot \frac{453.4}{144.6} \cdot \frac{28.4}{10.64} \cdot 0.996=0.96
$$

\section{Purity: 96\%}


S12. IMes.HPF ${ }_{6}{ }^{1} \mathrm{H}$ qNMR verifié !!!

\begin{tabular}{|l|l|l|l|}
\hline Zero filling & LB & Phase & Baseline \\
\hline $256 \mathrm{~K}$ & 0.1 & Manual & $6^{\text {Th }}$ order \\
\hline
\end{tabular}

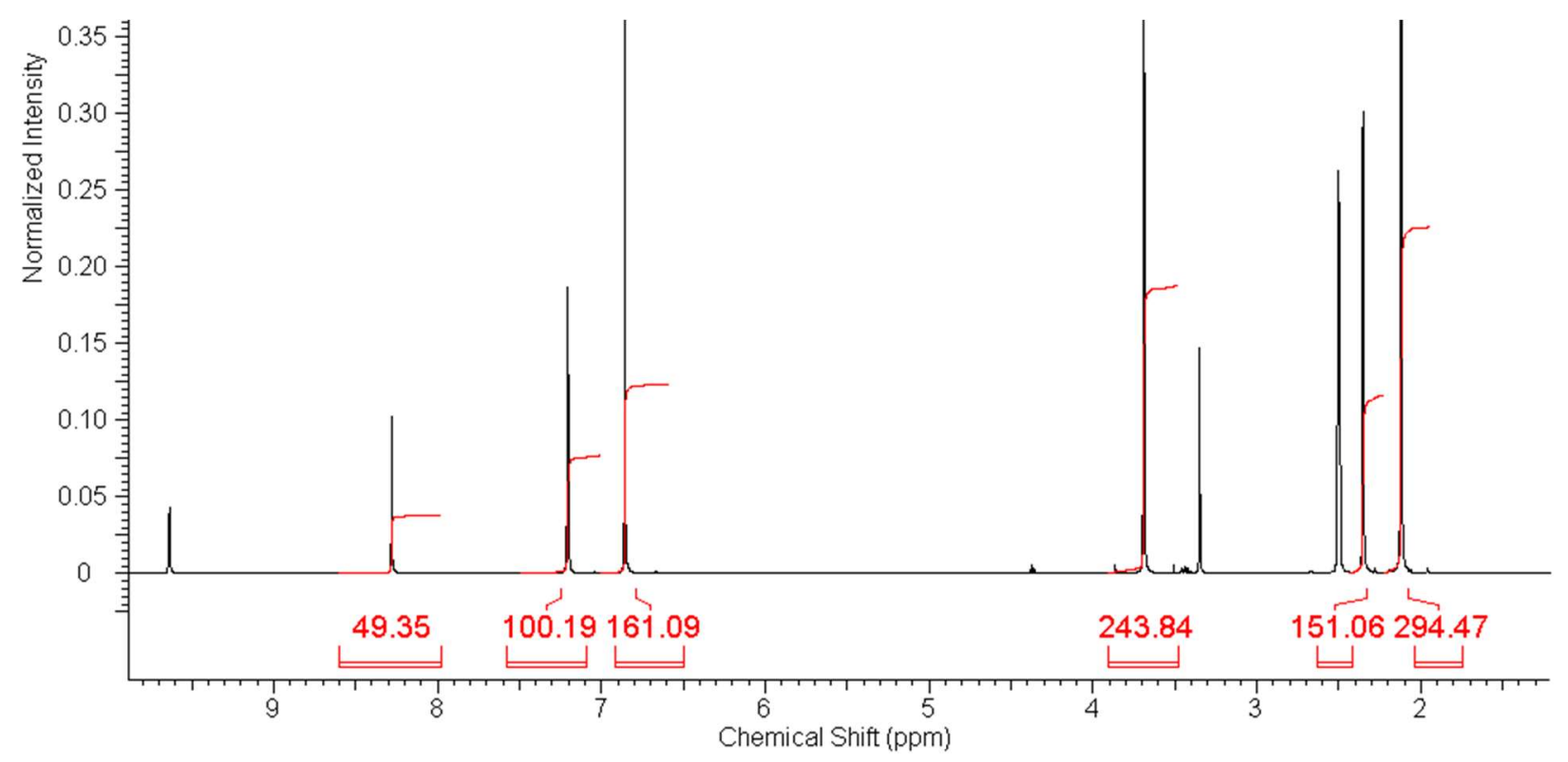

\begin{tabular}{|l|l|l|}
\hline Masse sample (mg) & Masse Standard (mg) & Solvent: dmso- $\mathrm{d}_{6}$ \\
\hline 31.51 & 15.25 & \\
\hline
\end{tabular}

\begin{tabular}{|l|l|l|l|l|}
\hline Signals standard (ppm) & 6.86 & 3.7 & & \\
\hline Integration & 161.1 & 243.8 & & \\
\hline \hline Signals sample (ppm) & 8.28 & 7.2 & 2.34 & 2.12 \\
\hline Integration & 49.35 & 100.2 & 151.1 & 294.5 \\
\hline
\end{tabular}

Somme of the integral: sample, 595.2; standard, 404.9.

Number of protons: sample, 24; standard, 10.

Molecular weight: sample, 450.4; standard, 138.2.

Mass of: sample, 31.51; standard: 15.25.

$$
P_{s}=\frac{I_{s}}{I_{s t d}} \cdot \frac{N_{s t d}}{N_{s}} \cdot \frac{M_{s}}{M_{s t d}} \cdot \frac{m_{s t d}}{m_{s}} \cdot P_{s t d}=\frac{595.2}{404.9} \cdot \frac{10}{24} \cdot \frac{450.4}{138.2} \cdot \frac{15.25}{31.51} \cdot 0.995=0.96
$$

\section{Purity: 96\%}




\section{S13. IMes.HPF ${ }_{6}{ }^{19} \mathrm{~F}$ qNMR Verifié!}

\begin{tabular}{|l|l|l|l|}
\hline Zero filling & LB & Phase & Baseline \\
\hline $256 \mathrm{~K}$ & 0.1 & Manual & $6^{\text {Th }}$ order \\
\hline
\end{tabular}

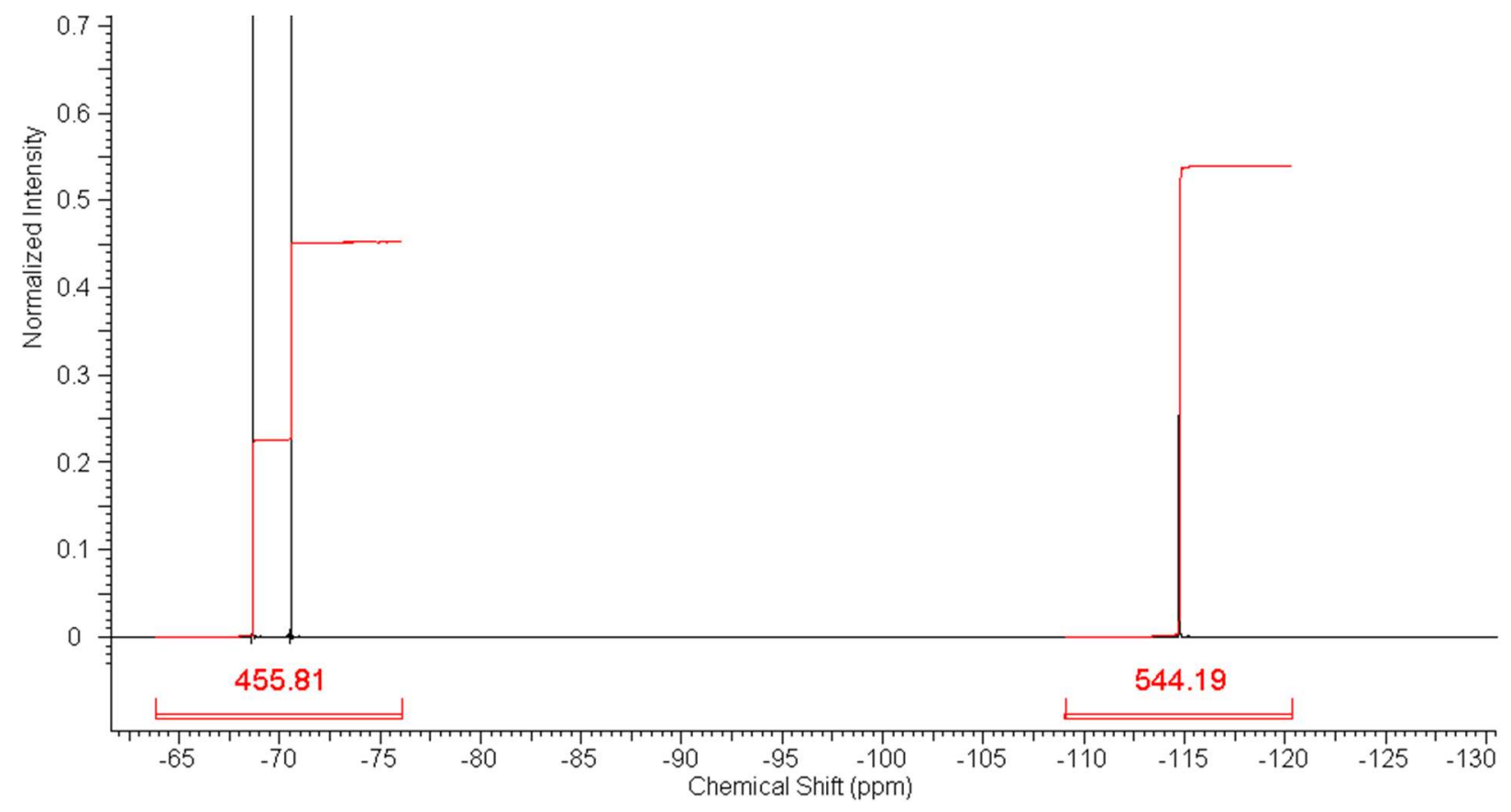

\begin{tabular}{|l|l|l|}
\hline Masse sample $(\mathrm{mg})$ & Masse Standard (mg) & Solvent: dmso- $\mathrm{d}_{6}$ \\
\hline 11.27 & 25.82 & \\
\hline
\end{tabular}

\begin{tabular}{|l|l|}
\hline Signals standard $(\mathrm{ppm})$ & -115 \\
\hline Integration & 544.2 \\
\hline \hline Signals sample $(\mathrm{ppm})$ & -70 \\
\hline Integration & 455.8 \\
\hline
\end{tabular}

Somme of the integral: sample, 455.8; standard, 544.2.

Number of fluorine: sample, 6; standard, 1.

Molecular weight: sample, 450.4; standard, 144.6.

Mass of: sample, 11.27; standard: 25.82.

$$
P_{s}=\frac{I_{s}}{I_{s t d}} \cdot \frac{N_{s t d}}{N_{s}} \cdot \frac{M_{s}}{M_{s t d}} \cdot \frac{m_{s t d}}{m_{s}} \cdot P_{s t d}=\frac{455.8}{544.2} \cdot \frac{1}{6} \cdot \frac{450.4}{144.6} \cdot \frac{25.82}{11.27} \cdot 0.996=0.99
$$

\section{Purity: 99\%}


S14. SIPr.HPF ${ }_{6}{ }^{1} \mathrm{H}$ qNMR Vérifié !!!

\begin{tabular}{|l|l|l|l|}
\hline Zero filling & LB & Phase & Baseline \\
\hline $256 \mathrm{~K}$ & 0.1 & Manual & $6^{\text {Th }}$ order \\
\hline
\end{tabular}

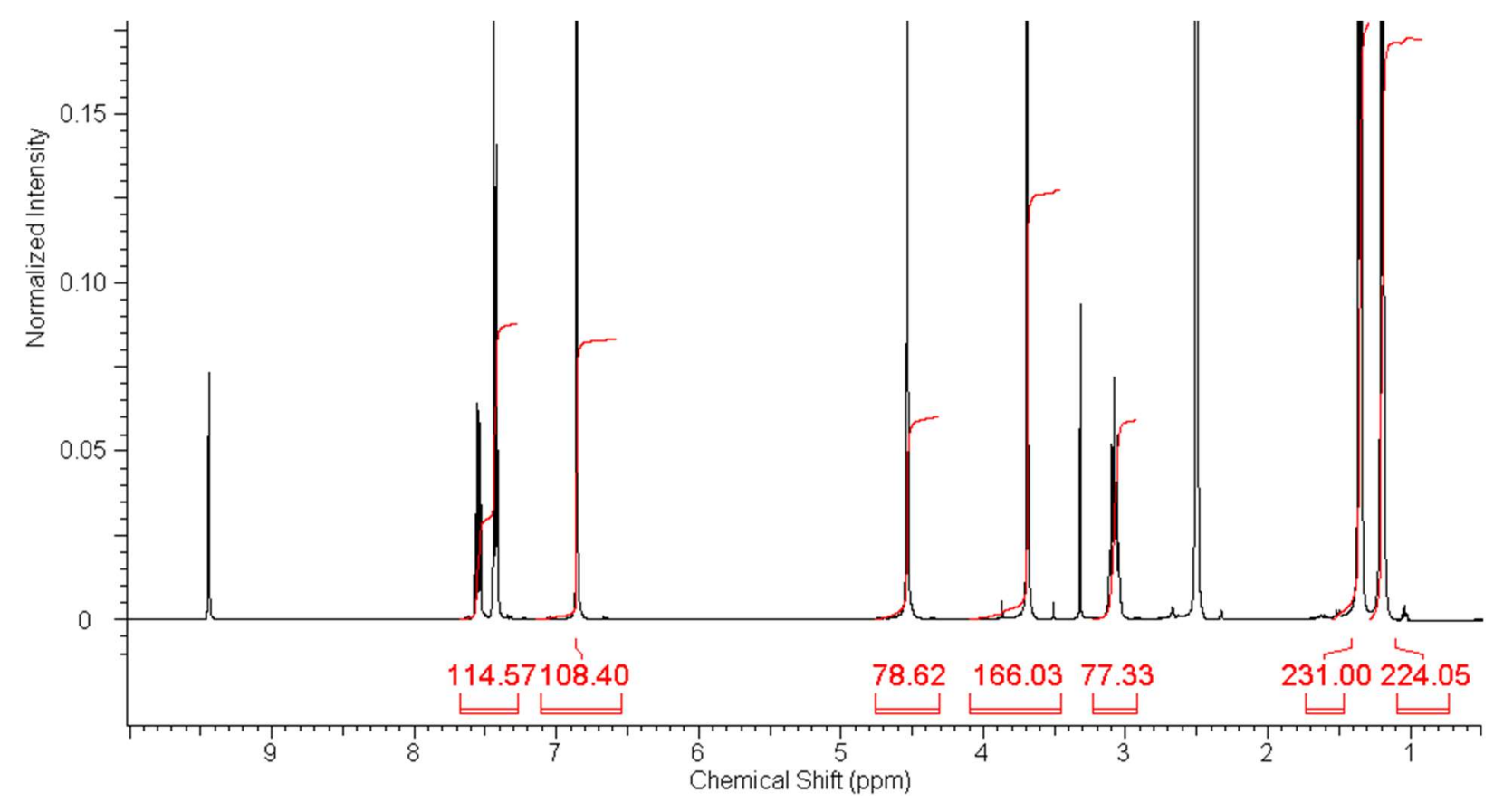

\begin{tabular}{|l|l|l|}
\hline Masse sample $(\mathrm{mg})$ & Masse Standard $(\mathrm{mg})$ & Solvent: dmso-d $\mathrm{d}_{6}$ \\
\hline 32.09 & 11.51 & \\
\hline
\end{tabular}

\begin{tabular}{|l|l|l|l|l|l|}
\hline Signals standard (ppm) & 6.86 & 3.7 & & & \\
\hline Integration & 108.4 & 166 & & & \\
\hline \hline Signals sample (ppm) & 7.49 & 4.54 & 3.1 & 11.35 & 1.19 \\
\hline Integration & 114.6 & 78.6 & 77.3 & 231 & 224.1 \\
\hline
\end{tabular}

Somme of the integral: sample, 725.6; standard, 274.4 .

Number of protons: sample, 38; standard, 10.

Molecular weight: sample, 536.6; standard, 138.2.

Mass of: sample, 32.09; standard: 11.51.

$$
P_{s}=\frac{I_{s}}{I_{s t d}} \cdot \frac{N_{s t d}}{N_{s}} \cdot \frac{M_{s}}{M_{s t d}} \cdot \frac{m_{s t d}}{m_{s}} \cdot P_{s t d}=\frac{725.6}{274.4} \cdot \frac{10}{38} \cdot \frac{536.6}{138.2} \cdot \frac{11.51}{32.09} \cdot 0.995=0.96
$$

\section{Purity: $96 \%$}




\section{S15. SIPr.HPF ${ }_{6}{ }^{19} \mathrm{~F}$ qNMR Vérifié}

\begin{tabular}{|l|l|l|l|}
\hline Zero filling & LB & Phase & Baseline \\
\hline $256 \mathrm{~K}$ & 0.1 & Manual & $6^{\text {Th }}$ order \\
\hline
\end{tabular}

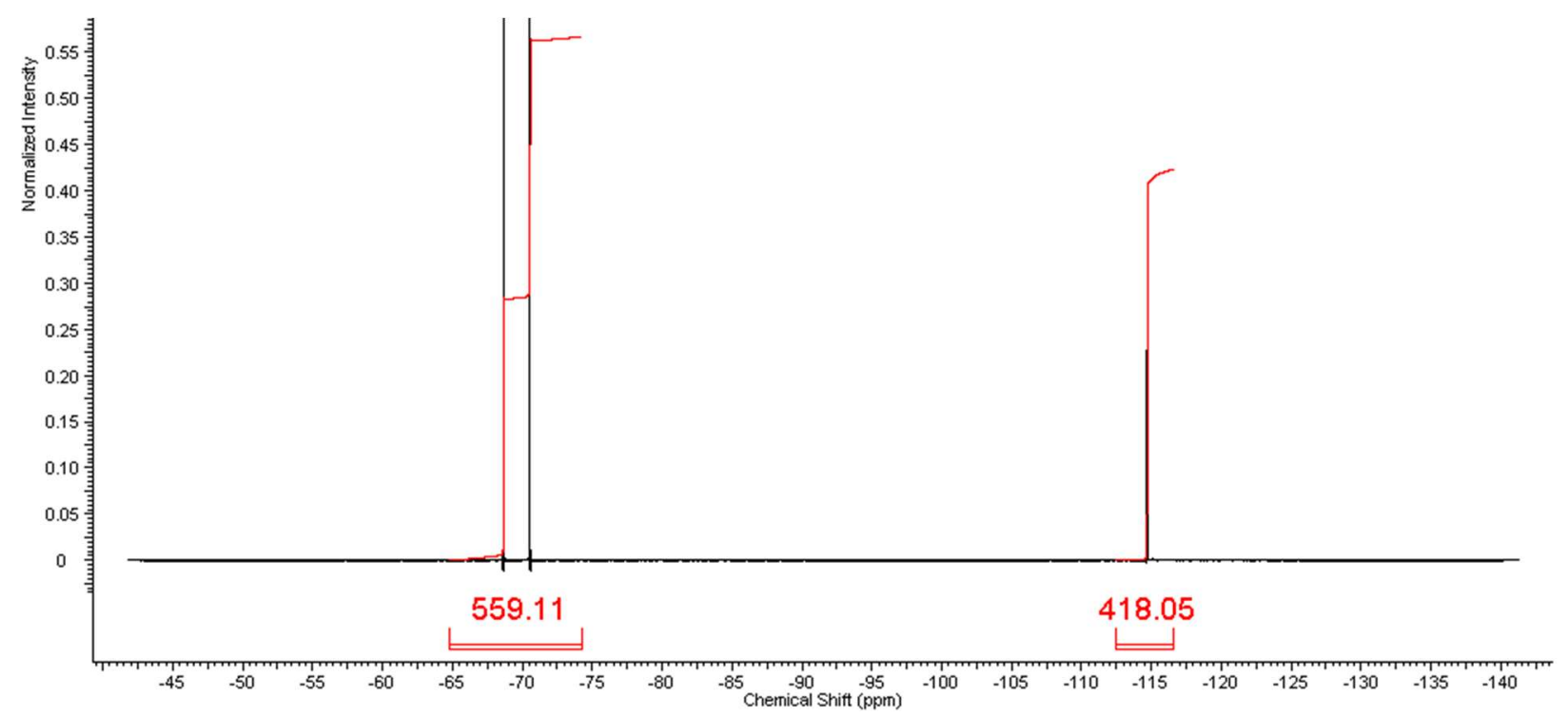

\begin{tabular}{|l|l|l|}
\hline Masse sample (mg) & Masse Standard (mg) & Solvent: dmso- $\mathrm{d}_{6}$ \\
\hline 11.56 & 13.6 & \\
\hline
\end{tabular}

\begin{tabular}{|l|l|}
\hline Signals standard $(\mathrm{ppm})$ & -115 \\
\hline Integration & 418.1 \\
\hline \hline Signals sample $(\mathrm{ppm})$ & -70 \\
\hline Integration & 559.1 \\
\hline
\end{tabular}

Somme of the integral: sample, 559.1; standard, 418.1.

Number of fluorine: sample, 6; standard, 1.

Molecular weight: sample, 534.6; standard, 144.6.

Mass of: sample, 11.56; standard: 13.6.

$$
P_{s}=\frac{I_{s}}{I_{s t d}} \cdot \frac{N_{s t d}}{N_{s}} \cdot \frac{M_{s}}{M_{s t d}} \cdot \frac{m_{s t d}}{m_{s}} \cdot P_{s t d}=\frac{559.1}{418.1} \cdot \frac{1}{6} \cdot \frac{534.6}{144.6} \cdot \frac{13.6}{11.56} \cdot 0.996=0.97
$$

\section{Purity: 97\%}


S16. IPr.HPF ${ }_{6}{ }^{1} \mathrm{H}$ qNMR Verifié!!

\begin{tabular}{|l|l|l|l|}
\hline Zero filling & LB & Phase & Baseline \\
\hline $256 \mathrm{~K}$ & 0.1 & Manual & $6^{\text {Th }}$ order \\
\hline
\end{tabular}

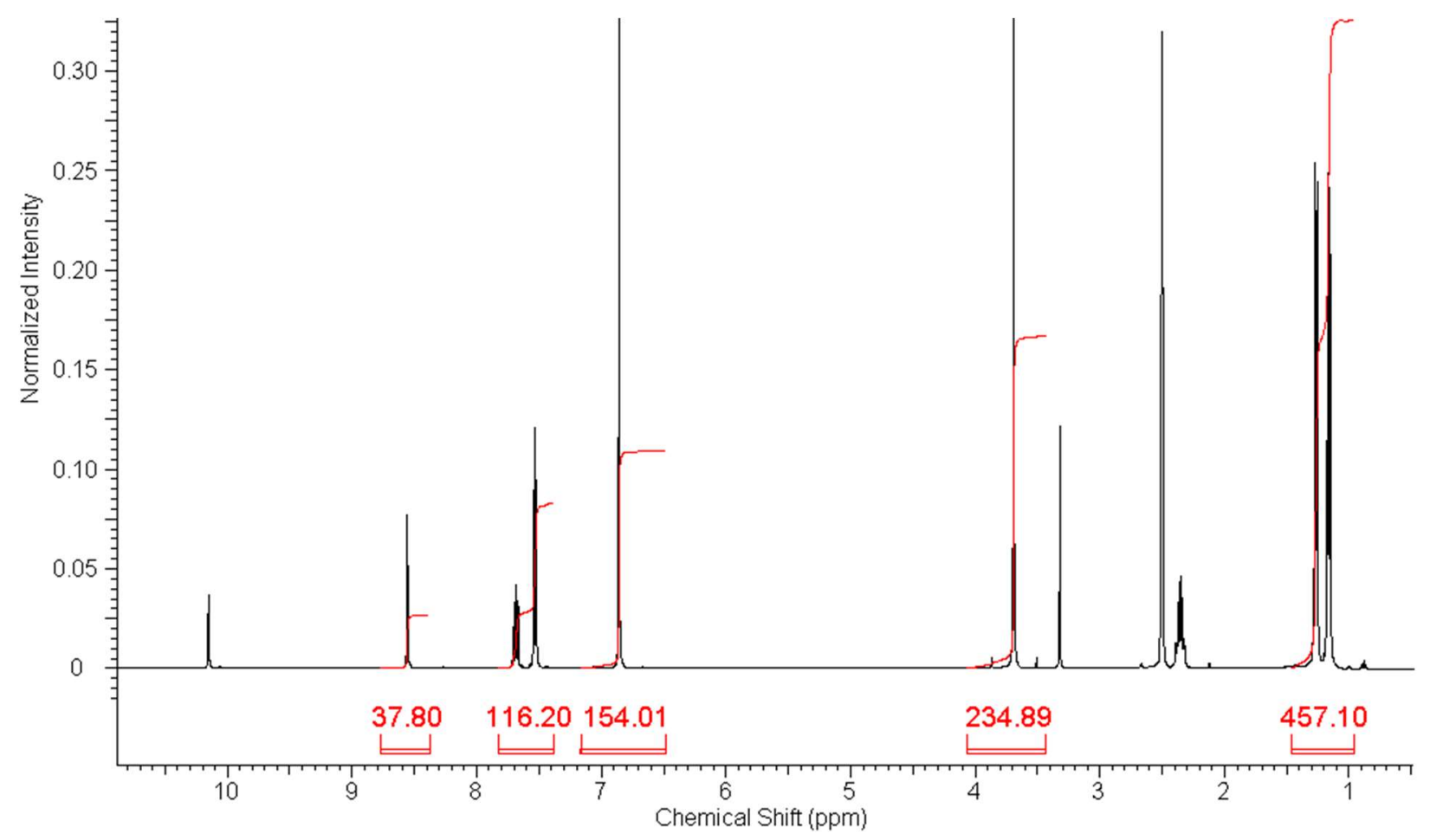

\begin{tabular}{|l|l|l|}
\hline Masse sample (mg) & Masse Standard (mg) & Solvent: dmso- $\mathrm{d}_{6}$ \\
\hline 41.33 & 20.79 & \\
\hline
\end{tabular}

\begin{tabular}{|l|l|l|l|}
\hline Signals standard $(\mathrm{ppm})$ & 6.86 & 3.7 & \\
\hline Integration & 154.0 & 234.9 & \\
\hline \hline Signals sample (ppm) & 8.56 & 7.61 & 1.21 \\
\hline Integration & 37.8 & 116.2 & 457.1 \\
\hline
\end{tabular}

Somme of the integral: sample, 611.1; standard, 388.9.

Number of protons: sample, 32; standard, 10.

Molecular weight: sample, 536.6; standard, 138.2.

Mass of: sample, 41.33; standard: 20.79.

$$
P_{s}=\frac{I_{s}}{I_{s t d}} \cdot \frac{N_{s t d}}{N_{s}} \cdot \frac{M_{s}}{M_{s t d}} \cdot \frac{m_{s t d}}{m_{s}} \cdot P_{s t d}=\frac{611.1}{388.9} \cdot \frac{10}{32} \cdot \frac{536.6}{138.2} \cdot \frac{20.79}{41.33} \cdot 0.995=0.95
$$

\section{Purity: 95\%}




\section{S17. IPr.HPF $6{ }^{19}$ F qNMR Verifié}

\begin{tabular}{|l|l|l|l|}
\hline Zero filling & LB & Phase & Baseline \\
\hline $256 \mathrm{~K}$ & 0.1 & Manual & $6^{\text {Th }}$ order \\
\hline
\end{tabular}

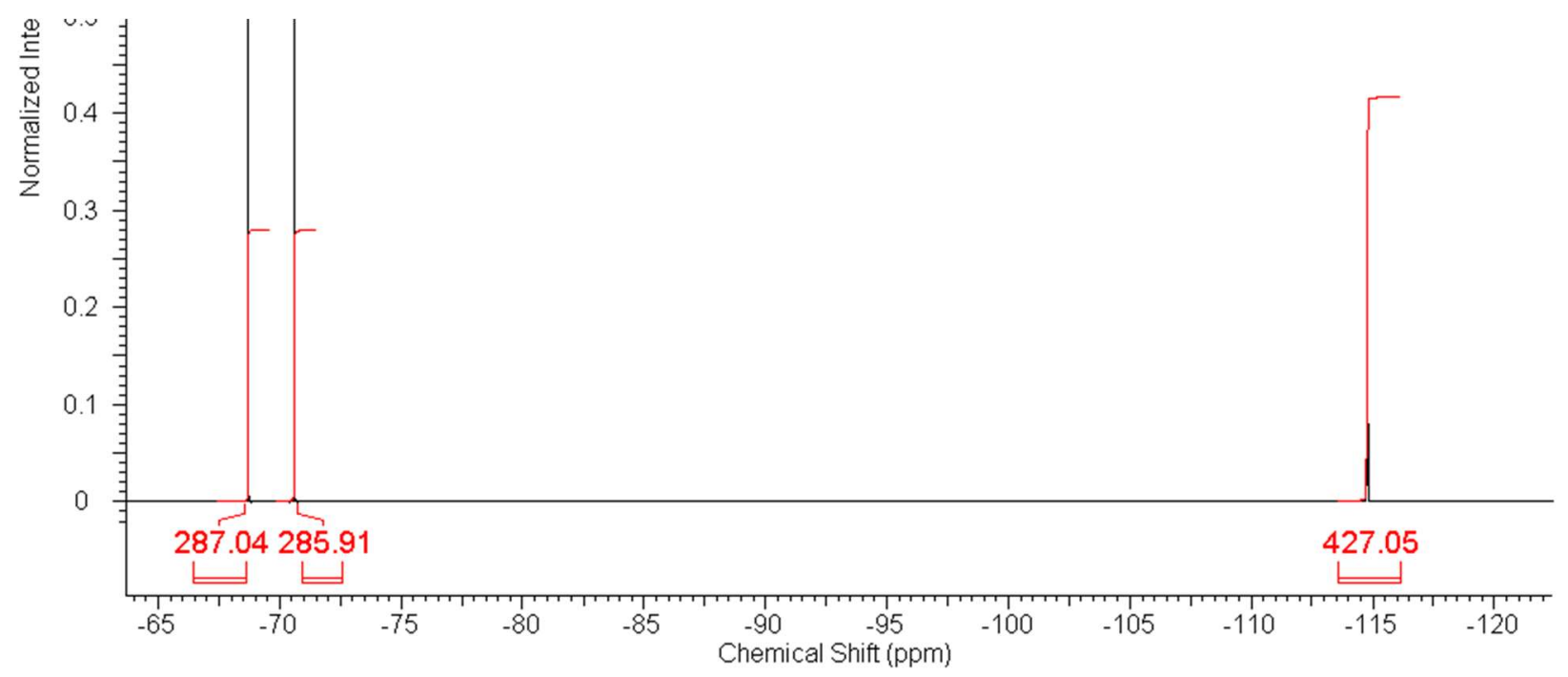

\begin{tabular}{|l|l|l|}
\hline Masse sample (mg) & Masse Standard (mg) & Solvent: dmso- $\mathrm{d}_{6}$ \\
\hline 12.80 & 14.98 & \\
\hline
\end{tabular}

\begin{tabular}{|l|l|l|}
\hline Signals standard $(\mathrm{ppm})$ & -115 & \\
\hline Integration & 427 & \\
\hline \hline Signals sample $(\mathrm{ppm})$ & -70 & -70 \\
\hline Integration & 287 & 285.9 \\
\hline
\end{tabular}

Somme of the integral: sample, 572.9; standard, 427.

Number of fluorine: sample, 6; standard, 1.

Molecular weight: sample, 534.6; standard, 144.6.

Mass of: sample, 12.8; standard: 14.98.

$$
P_{s}=\frac{I_{s}}{I_{s t d}} \cdot \frac{N_{s t d}}{N_{s}} \cdot \frac{M_{s}}{M_{s t d}} \cdot \frac{m_{s t d}}{m_{s}} \cdot P_{s t d}=\frac{572.9}{427} \cdot \frac{1}{6} \cdot \frac{534.6}{144.6} \cdot \frac{14.98}{12.8} \cdot 0.996=0.96
$$

Purity : 96\% 


\section{S18. SIMes.HBF ${ }_{4}{ }^{1} \mathrm{H}$ qNMR Verifié !!!}

\begin{tabular}{|l|l|l|l|}
\hline Zero filling & LB & Phase & Baseline \\
\hline $256 \mathrm{~K}$ & 0.1 & Manual & $6^{\text {Th }}$ order \\
\hline
\end{tabular}

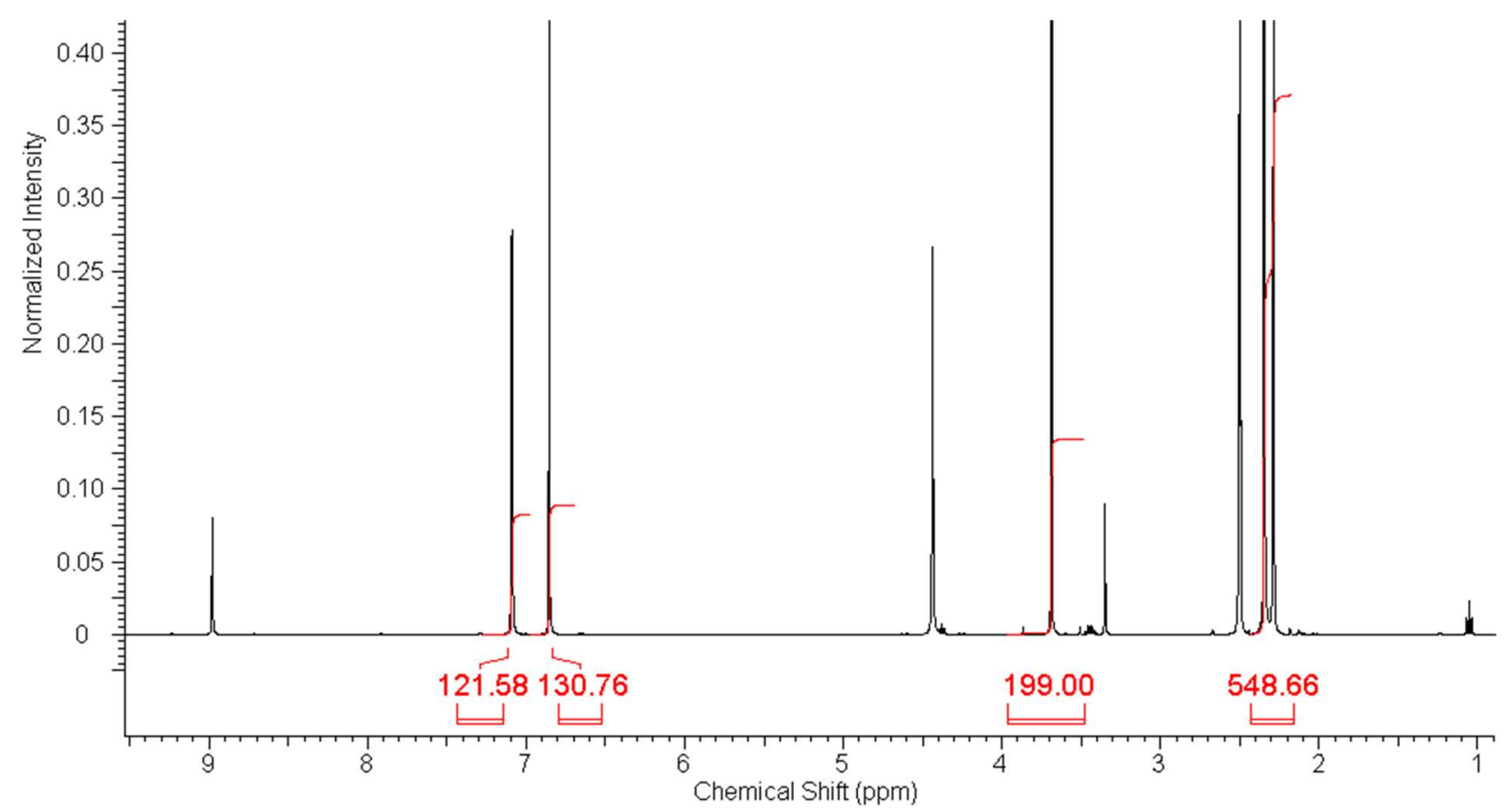

\begin{tabular}{|l|l|l|}
\hline Masse sample $(\mathrm{mg})$ & Masse Standard $(\mathrm{mg})$ & Solvent: dmso- $_{6}$ \\
\hline 31.18 & 11.75 & \\
\hline
\end{tabular}

\begin{tabular}{|l|l|l|}
\hline Signals standard $(\mathrm{ppm})$ & 6.86 & 3.7 \\
\hline Integration & 130.8 & 199 \\
\hline \hline Signals sample $(\mathrm{ppm})$ & 7.09 & 2.32 \\
\hline Integration & 121.6 & 548.7 \\
\hline
\end{tabular}

Somme of the integral: sample, 670.3 ; standard, 329.8 .

Number of protons: sample, 22; standard, 10.

Molecular weight: sample, 394.3; standard, 138.2.

Mass of: sample, 31.18; standard: 11.75.

$$
P_{s}=\frac{I_{s}}{I_{s t d}} \cdot \frac{N_{s t d}}{N_{s}} \cdot \frac{M_{s}}{M_{s t d}} \cdot \frac{m_{s t d}}{m_{s}} \cdot P_{s t d}=\frac{670.3}{329.8} \cdot \frac{10}{22} \cdot \frac{394.3}{138.2} \cdot \frac{11.75}{31.18} \cdot 0.995=0.98
$$

\section{Purity: 98\%}




\section{S19. SIMes.HBF $4{ }^{19} \mathrm{~F}$ qNMR Verifié}

\begin{tabular}{|l|l|l|l|}
\hline Zero filling & LB & Phase & Baseline \\
\hline $256 \mathrm{~K}$ & 0.1 & Manual & $6^{\text {Th }}$ order \\
\hline
\end{tabular}

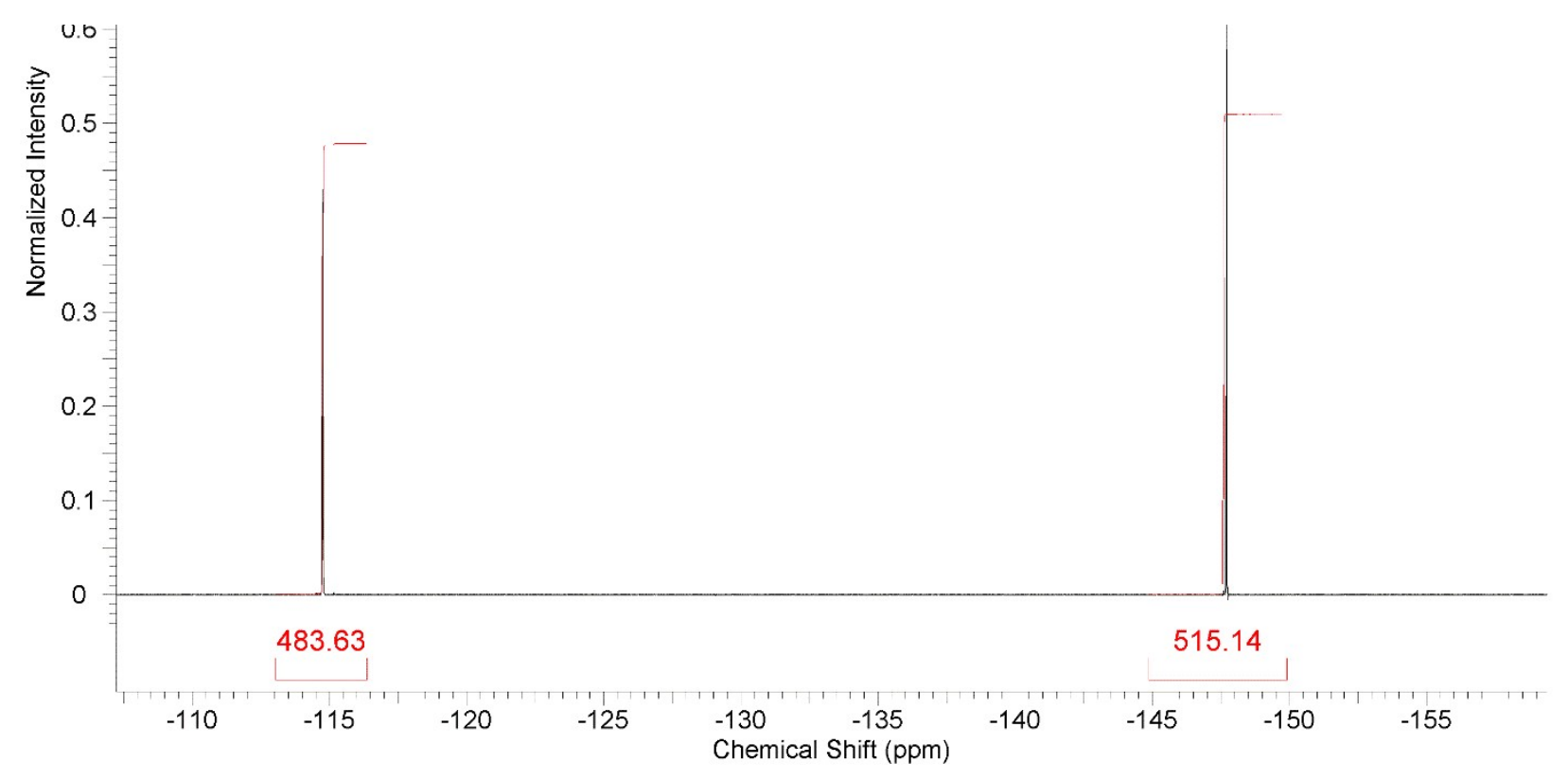

\begin{tabular}{|l|l|l|}
\hline Masse sample $(\mathrm{mg})$ & Masse Standard $(\mathrm{mg})$ & Solvent: $\mathrm{dmso}_{\mathrm{-}} \mathrm{d}_{6}$ \\
\hline 10.62 & 14.58 & \\
\hline
\end{tabular}

\begin{tabular}{|l|l|}
\hline Signals standard (ppm) & -115 \\
\hline Integration & 484 \\
\hline \hline Signals sample (ppm) & -148 \\
\hline Integration & 515 \\
\hline
\end{tabular}

Somme of the integral: sample, 515; standard, 484.

Number of fluorine: sample, 4; standard, 1.

Molecular weight: sample, 394.2; standard, 144.6.

Mass of: sample, 10.62; standard: 14.58.

$$
P_{s}=\frac{I_{s}}{I_{s t d}} \cdot \frac{N_{s t d}}{N_{s}} \cdot \frac{M_{s}}{M_{s t d}} \cdot \frac{m_{s t d}}{m_{s}} \cdot P_{s t d}=\frac{515}{484} \cdot \frac{1}{4} \cdot \frac{394.2}{144.6} \cdot \frac{14.58}{10.62} \cdot 0.996=0.99
$$

\section{Purity: 99\%}




\section{S20. IMes.HBF ${ }_{4}{ }^{1} \mathrm{H}$ qNMR Verifié !!}

\begin{tabular}{|l|l|l|l|}
\hline Zero filling & LB & Phase & Baseline \\
\hline $256 \mathrm{~K}$ & 0.1 & Manual & $6^{\mathrm{Th}}$ order \\
\hline
\end{tabular}

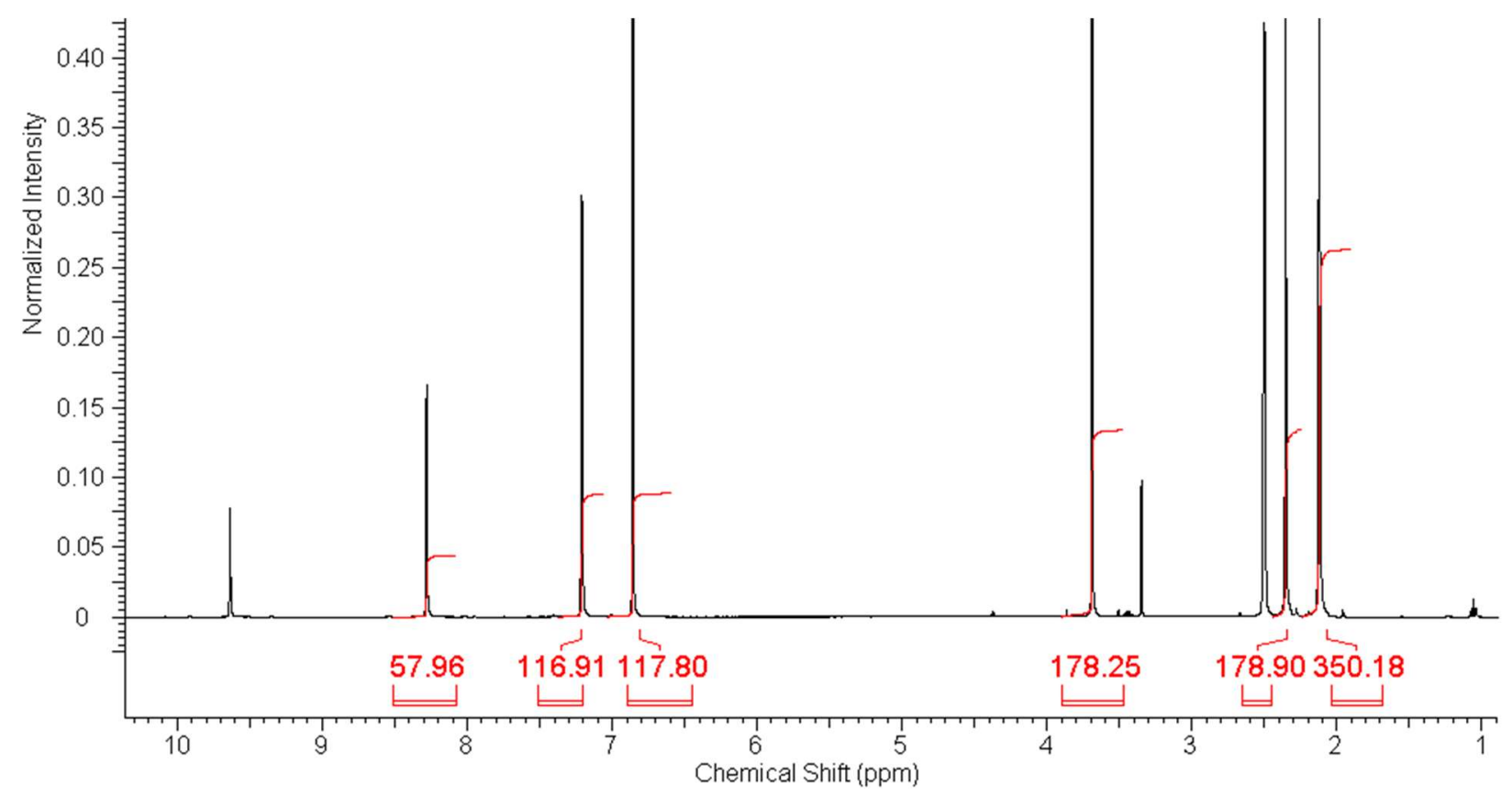

\begin{tabular}{|l|l|l|}
\hline Masse sample $(\mathrm{mg})$ & Masse Standard $(\mathrm{mg})$ & Solvent: $\mathrm{dmso}_{\mathrm{d}} \mathrm{d}_{6}$ \\
\hline 35.55 & 12.02 & \\
\hline
\end{tabular}

\begin{tabular}{|l|l|l|l|l|}
\hline Signals standard $(\mathrm{ppm})$ & 6.86 & 3.7 & & \\
\hline Integration & 117.8 & 178.3 & & \\
\hline \hline Signals sample (ppm) & 8.3 & 7.2 & 2.4 & 2.1 \\
\hline Integration & 58 & 116.9 & 178.9 & 350.2 \\
\hline
\end{tabular}

Somme of the integral: sample, 704; standard, 296.1.

Number of protons: sample, 24; standard, 10.

Molecular weight: sample, 392.2; standard, 138.2.

Mass of: sample, $35.55 ;$ standard: 12.02.

$$
P_{s}=\frac{I_{s}}{I_{s t d}} \cdot \frac{N_{s t d}}{N_{s}} \cdot \frac{M_{s}}{M_{s t d}} \cdot \frac{m_{s t d}}{m_{s}} \cdot P_{s t d}=\frac{704}{296.1} \cdot \frac{10}{24} \cdot \frac{392.2}{138.2} \cdot \frac{12.02}{35.55} \cdot 0.995=0.94
$$

\section{Purity: 94\%}




\section{S21. IMes.HBF ${ }_{4}{ }^{19} \mathrm{~F}$ qNMR Verifié}

\begin{tabular}{|l|l|l|l|}
\hline Zero filling & LB & Phase & Baseline \\
\hline $256 \mathrm{~K}$ & 0.1 & Manual & $6^{\text {Th }}$ order \\
\hline
\end{tabular}

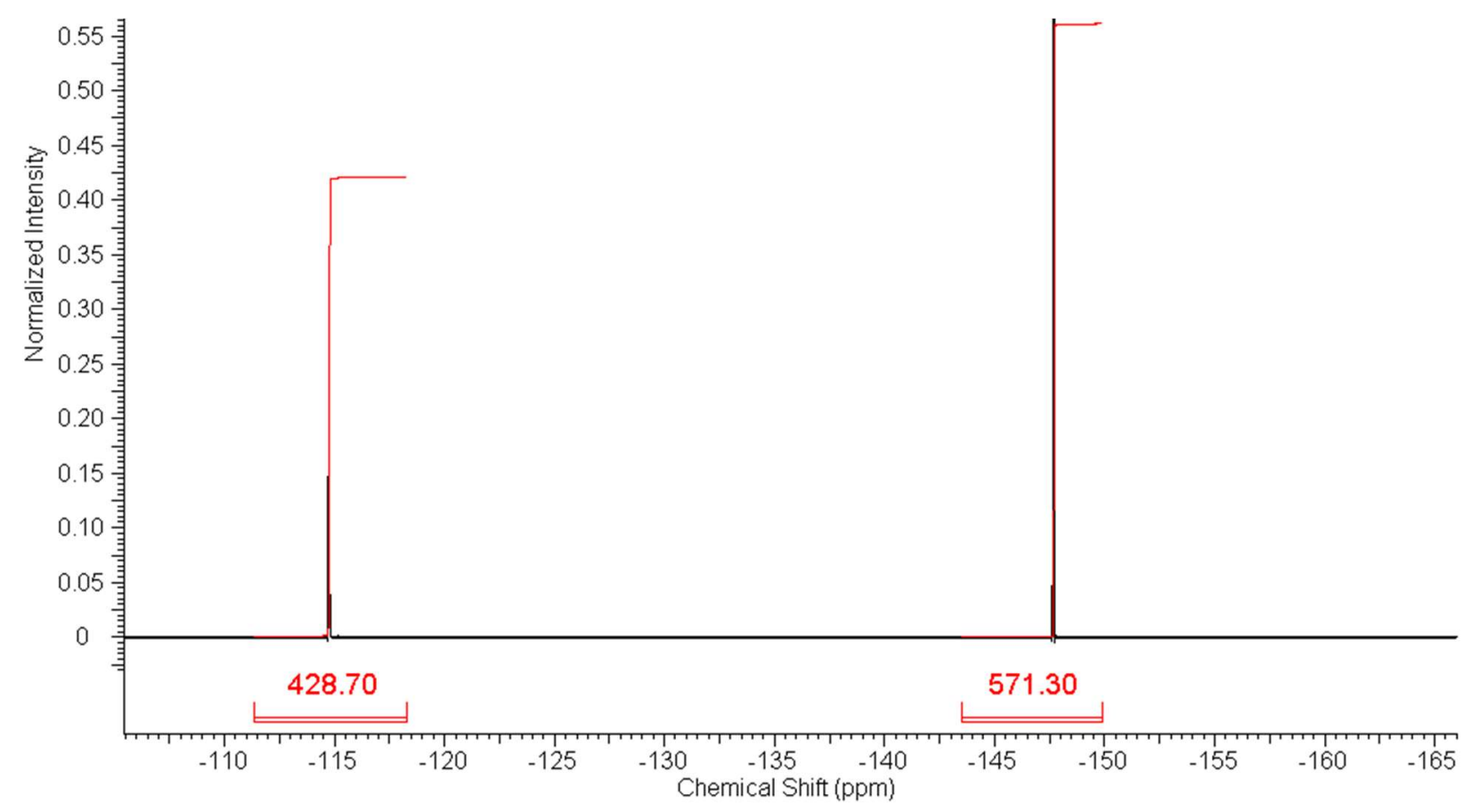

\begin{tabular}{|l|l|l|}
\hline Masse sample (mg) & Masse Standard (mg) & Solvent: dmso- $_{6}$ \\
\hline 11.18 & 12.1 & \\
\hline
\end{tabular}

\begin{tabular}{|l|l|}
\hline Signals standard (ppm) & -115 \\
\hline Integration & 429 \\
\hline \hline Signals sample (ppm) & -148 \\
\hline Integration & 571 \\
\hline
\end{tabular}

Somme of the integral: sample, 571; standard, 429.

Number of fluorine: sample, 4; standard, 1.

Molecular weight: sample, 392.2; standard, 144.6.

Mass of: sample, 11.18; standard: 12.1.

$$
P_{s}=\frac{I_{s}}{I_{s t d}} \cdot \frac{N_{s t d}}{N_{s}} \cdot \frac{M_{s}}{M_{s t d}} \cdot \frac{m_{s t d}}{m_{s}} \cdot P_{s t d}=\frac{571}{429} \cdot \frac{1}{4} \cdot \frac{392.2}{144.6} \cdot \frac{12.1}{11.18} \cdot 0.996=0.97
$$

\section{Purity: 97\%}




\section{S22. SIPr. $\mathrm{HBF}_{4}{ }^{1} \mathrm{H}$ qNMR Verifié!!}

\begin{tabular}{|l|l|l|l|}
\hline Zero filling & LB & Phase & Baseline \\
\hline $256 \mathrm{~K}$ & 0.1 & Manual & $6^{\mathrm{Th}}$ order \\
\hline
\end{tabular}

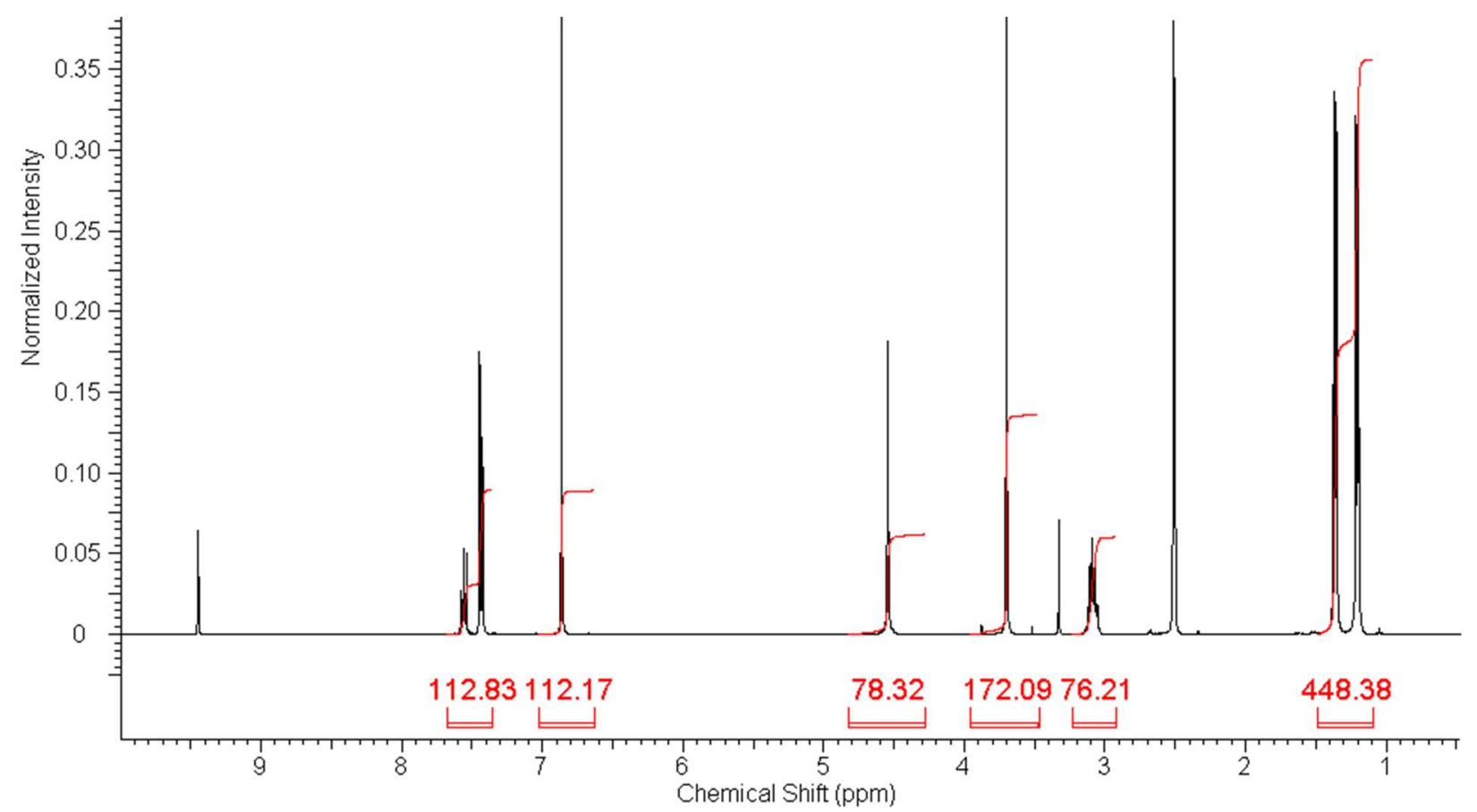

\begin{tabular}{|l|l|l|}
\hline Masse sample $(\mathrm{mg})$ & Masse Standard $(\mathrm{mg})$ & Solvent: $\mathrm{dmso}_{\mathrm{d}} \mathrm{d}_{6}$ \\
\hline 30.8 & 12.92 & \\
\hline
\end{tabular}

\begin{tabular}{|l|l|l|l|l|}
\hline Signals standard (ppm) & 6.86 & 3.7 & & \\
\hline Integration & 112.2 & 172.2 & & \\
\hline \hline Signals sample (ppm) & 7.5 & 4.6 & 3.1 & 1.29 \\
\hline Integration & 112.8 & 78.3 & 76.2 & 448.4 \\
\hline
\end{tabular}

Somme of the integral: sample, 715.7; standard, 284.4.

Number of protons: sample, 38; standard, 10.

Molecular weight: sample, 478.4; standard, 138.2.

Mass of: sample, 30.8; standard: 12.92.

$$
P_{s}=\frac{I_{s}}{I_{s t d}} \cdot \frac{N_{s t d}}{N_{s}} \cdot \frac{M_{s}}{M_{s t d}} \cdot \frac{m_{s t d}}{m_{s}} \cdot P_{s t d}=\frac{715.7}{284.4} \cdot \frac{10}{38} \cdot \frac{478.4}{138.2} \cdot \frac{12.92}{30.8} \cdot 0.995=0.95
$$

\section{Purity: 95\%}




\section{S23. SIPr. $\mathrm{HBF}_{4},{ }^{19} \mathrm{~F}$ qNMR Verifié}

\begin{tabular}{|l|l|l|l|}
\hline Zero filling & LB & Phase & Baseline \\
\hline $256 \mathrm{~K}$ & 0.1 & Manual & $6^{\mathrm{Th}}$ order \\
\hline
\end{tabular}

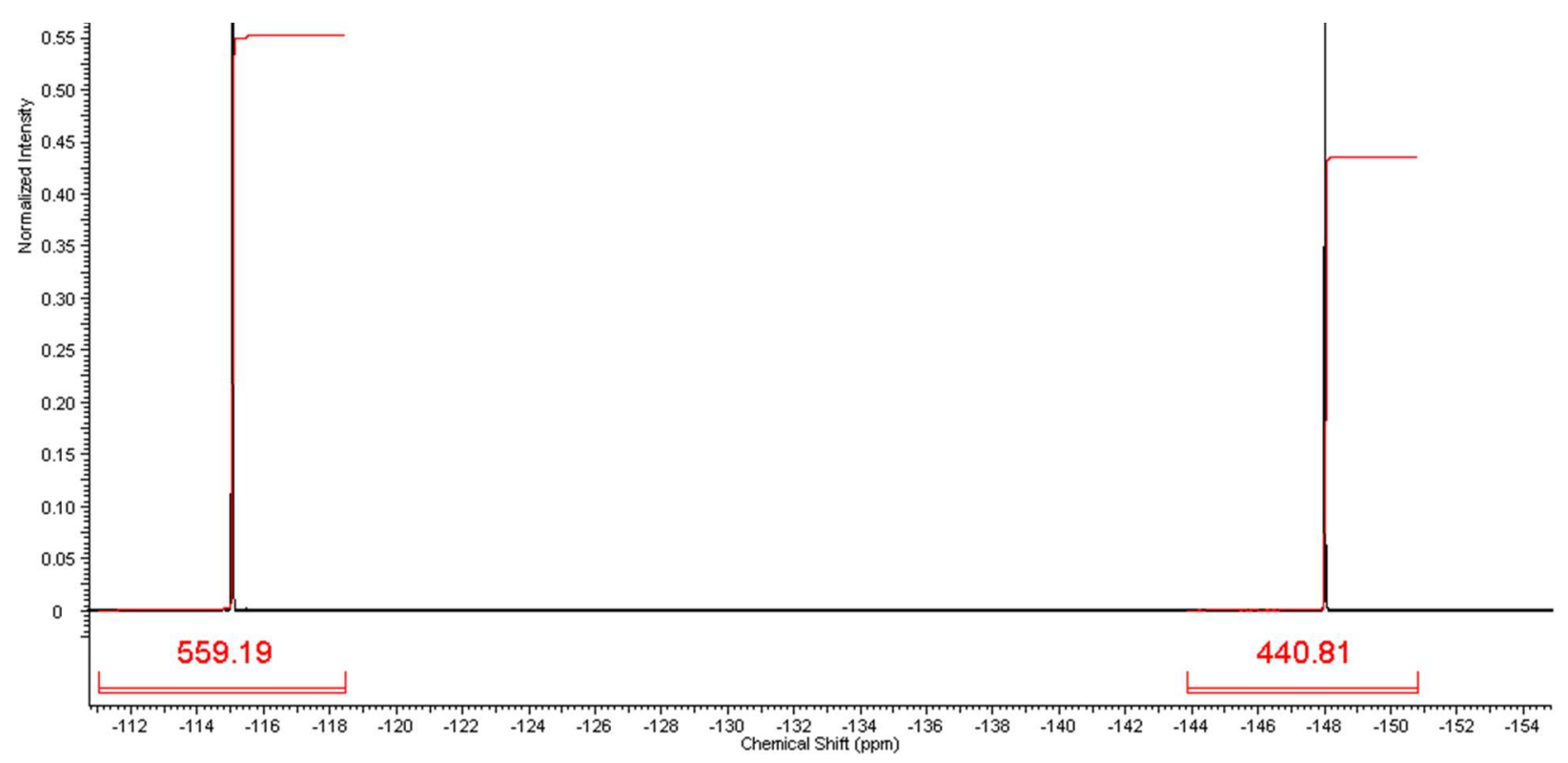

\begin{tabular}{|l|l|l|}
\hline Masse sample $(\mathrm{mg})$ & Masse Standard (mg) & Solvent: dmso- $_{6}$ \\
\hline 10.1 & 15.2 & \\
\hline
\end{tabular}

\begin{tabular}{|l|l|}
\hline Signals standard $(\mathrm{ppm})$ & -115 \\
\hline Integration & 559.2 \\
\hline \hline Signals sample $(\mathrm{ppm})$ & -148 \\
\hline Integration & 440.8 \\
\hline
\end{tabular}

Somme of the integral: sample, 440.8; standard, 559.2.

Number of fluorine: sample, 4; standard, 1.

Molecular weight: sample, 478.4; standard, 144.6.

Mass of: sample, 10.1; standard: 15.2.

$$
P_{s}=\frac{I_{s}}{I_{s t d}} \cdot \frac{N_{s t d}}{N_{s}} \cdot \frac{M_{s}}{M_{s t d}} \cdot \frac{m_{s t d}}{m_{s}} \cdot P_{s t d}=\frac{440.8}{559.2} \cdot \frac{1}{4} \cdot \frac{478.4}{144.6} \cdot \frac{15.2}{10.1} \cdot 0.996=0.97
$$

\section{Purity: 97\%}




\section{S24. IPr. $\mathrm{HBF}_{4},{ }^{1} \mathrm{H}$ qNMR Verifié!!!}

\begin{tabular}{|l|l|l|l|}
\hline Zero filling & LB & Phase & Baseline \\
\hline $256 \mathrm{~K}$ & 0.1 & Manual & $6^{\mathrm{Th}}$ order \\
\hline
\end{tabular}

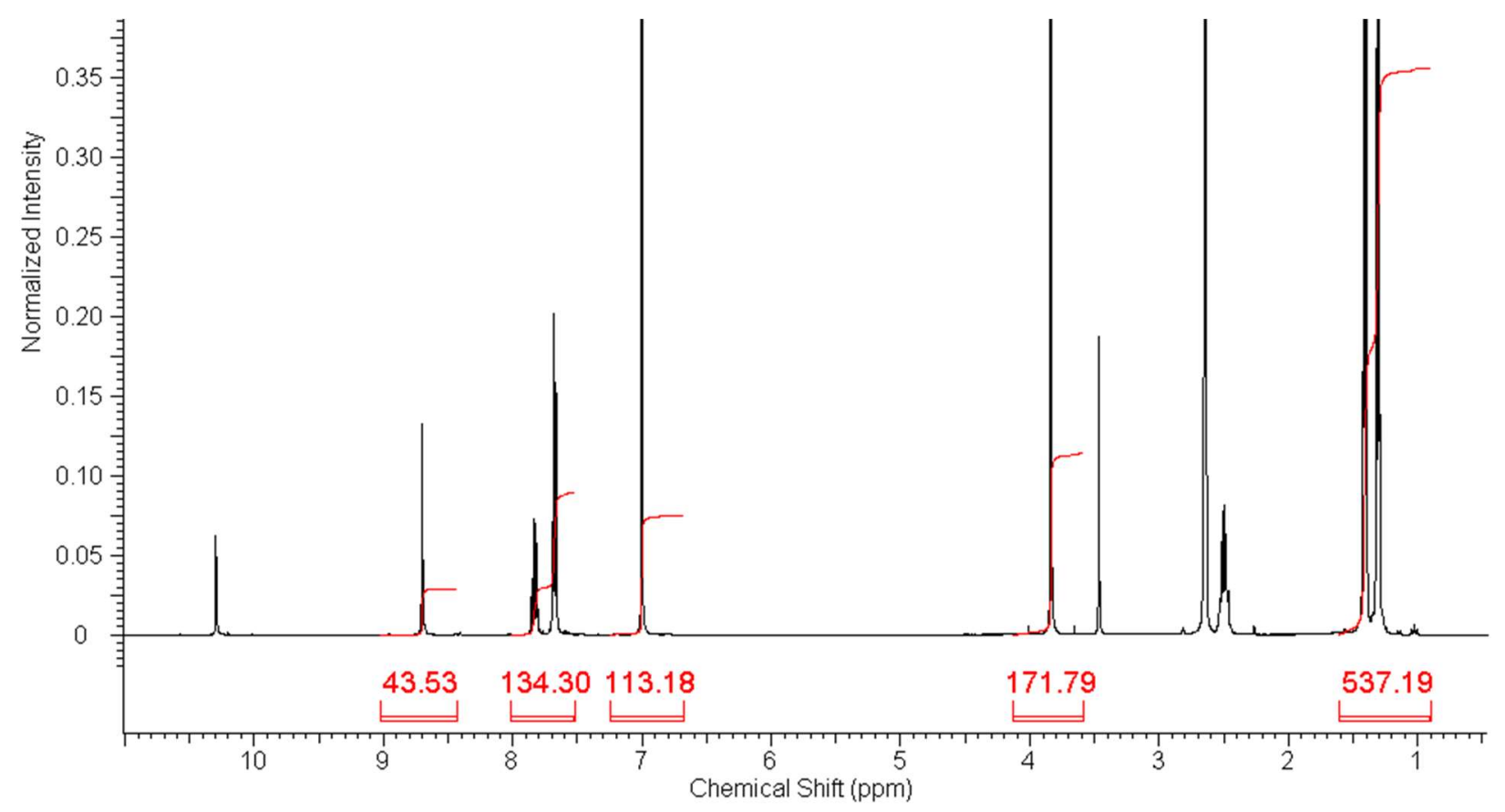

\begin{tabular}{|l|l|l|}
\hline Masse sample $(\mathrm{mg})$ & Masse Standard (mg) & Solvent: $\mathrm{dmso}_{\mathrm{d}} \mathrm{d}_{6}$ \\
\hline 31.58 & 10.96 & \\
\hline
\end{tabular}

\begin{tabular}{|l|l|l|l|}
\hline Signals standard (ppm) & 6.86 & 3.7 & \\
\hline Integration & 113.2 & 171.8 & \\
\hline \hline Signals sample (ppm) & 8.7 & 7.8 & 1.4 \\
\hline Integration & 45.53 & 134.3 & 537.2 \\
\hline
\end{tabular}

Somme of the integral: sample, 717.3; standard, 285.

Number of protons: sample, 32; standard, 10.

Molecular weight: sample, 476.4; standard, 138.2.

Mass of: sample, 31.58; standard: 10.96.

$$
P_{s}=\frac{I_{s}}{I_{s t d}} \cdot \frac{N_{s t d}}{N_{s}} \cdot \frac{M_{s}}{M_{s t d}} \cdot \frac{m_{s t d}}{m_{s}} \cdot P_{s t d}=\frac{717.3}{285} \cdot \frac{10}{32} \cdot \frac{476.4}{138.2} \cdot \frac{10.96}{31.58} \cdot 0.995=0.93
$$

Purity: 93\% 


\section{S25. IPr. $\mathrm{HBF}_{4},{ }^{19} \mathrm{~F}$ qNMR Verifié!!}

\begin{tabular}{|l|l|l|l|}
\hline Zero filling & LB & Phase & Baseline \\
\hline $256 \mathrm{~K}$ & 0.1 & Manual & $6^{\mathrm{Th}}$ order \\
\hline
\end{tabular}

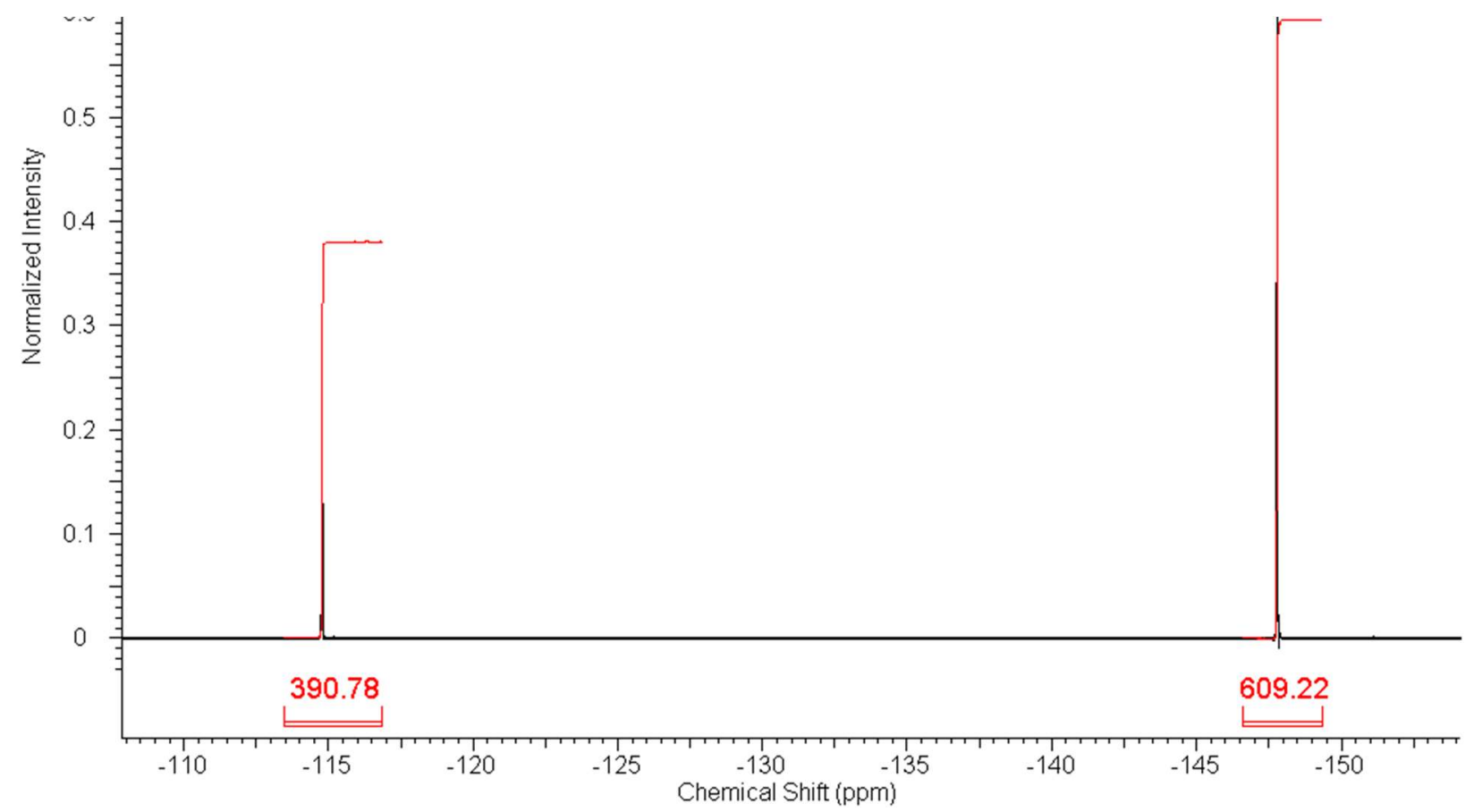

\begin{tabular}{|l|l|l|}
\hline Masse sample $(\mathrm{mg})$ & Masse Standard $(\mathrm{mg})$ & Solvent: $\mathrm{dmso}_{6}-\mathrm{d}_{6}$ \\
\hline 14.9 & 11.2 & \\
\hline
\end{tabular}

\begin{tabular}{|l|l|}
\hline Signals standard $(\mathrm{ppm})$ & -115 \\
\hline Integration & 390.8 \\
\hline \hline Signals sample (ppm) & -148 \\
\hline Integration & 609.2 \\
\hline
\end{tabular}

Somme of the integral: sample, 609.2; standard, 390.8.

Number of fluorine: sample, 4; standard, 1.

Molecular weight: sample, 476.4; standard, 144.6.

Mass of: sample, 14.9; standard: 11.12.

$$
P_{s}=\frac{I_{s}}{I_{s t d}} \cdot \frac{N_{s t d}}{N_{s}} \cdot \frac{M_{s}}{M_{s t d}} \cdot \frac{m_{s t d}}{m_{s}} \cdot P_{s t d}=\frac{609.2}{390.8} \cdot \frac{1}{4} \cdot \frac{476.4}{144.6} \cdot \frac{11.12}{14.9} \cdot 0.996=0.95
$$

\section{Purity: 95\%}




\section{S26. IMesAgCl, ${ }^{1} \mathrm{H}$ qNMR Vérifié!!!}

\begin{tabular}{|l|l|l|l|}
\hline Zero filling & LB & Phase & Baseline \\
\hline $256 \mathrm{~K}$ & 0.1 & Manual & $6^{\text {Th }}$ order \\
\hline
\end{tabular}

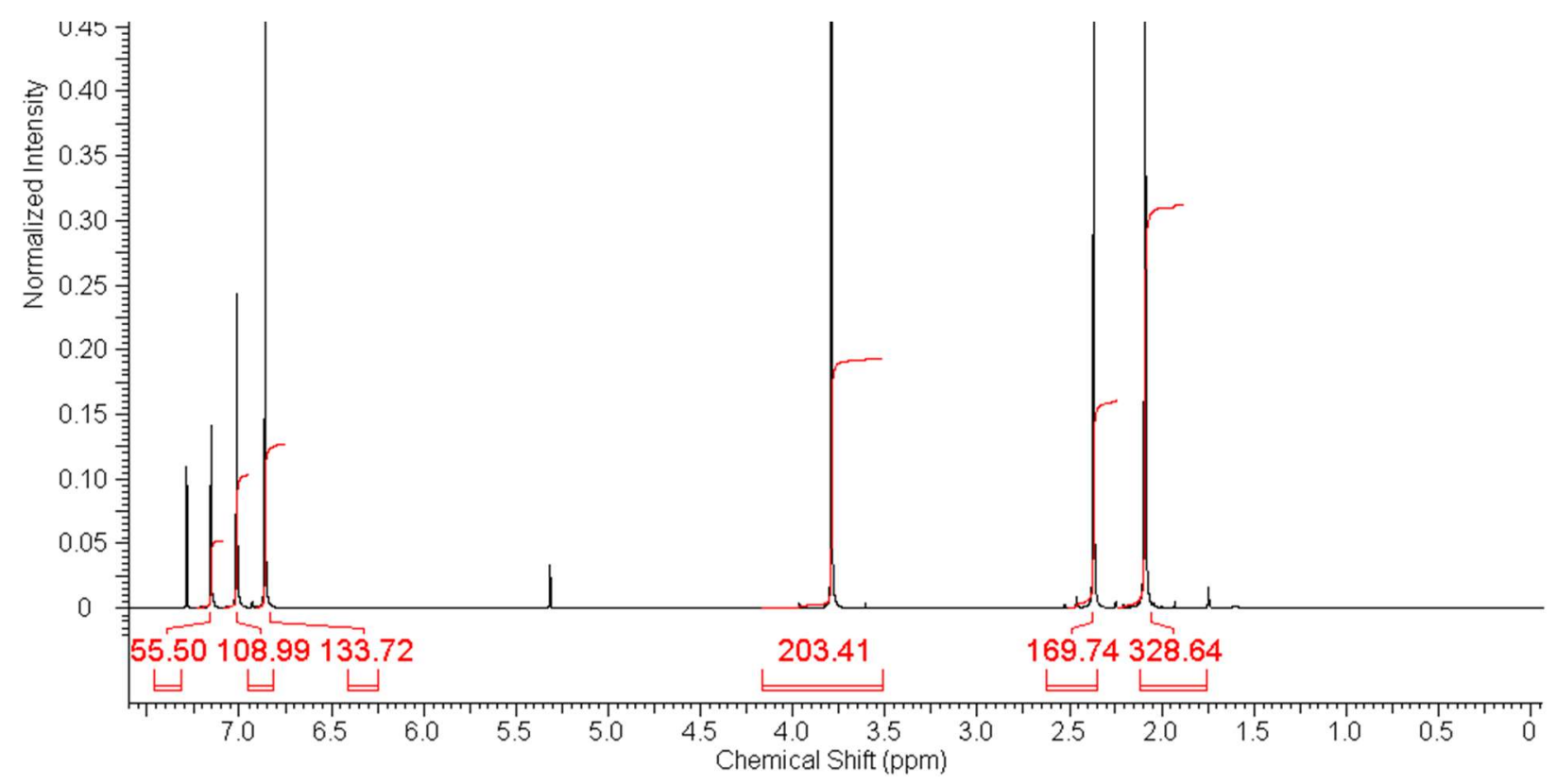

\begin{tabular}{|l|l|l|}
\hline Masse sample (mg) & Masse Standard (mg) & Solvent: $\mathrm{CDCl}_{3}$ \\
\hline 41.72 & 14.9 & \\
\hline
\end{tabular}

\begin{tabular}{|c|c|c|c|c|}
\hline Signals standard (ppm) & 6.86 & 3.7 & & \\
\hline Integration & 133.72 & 203.4 & & \\
\hline Signals sample (ppm) & 7.14 & 7.0 & 2.36 & 2.07 \\
\hline Integration & 55.5 & 109 & 169.7 & 328.6 \\
\hline
\end{tabular}

Somme of the integral: sample, $662.8 ;$ standard, 327.1.

Number of protons: sample, 24; standard, 10.

Molecular weight: sample, 449.7; standard, 138.2.

Mass of: sample, 41.72; standard: 14.9.

$$
P_{s}=\frac{I_{s}}{I_{s t d}} \cdot \frac{N_{s t d}}{N_{s}} \cdot \frac{M_{s}}{M_{s t d}} \cdot \frac{m_{s t d}}{m_{s}} \cdot P_{s t d}=\frac{662.8}{337.4} \cdot \frac{10}{24} \cdot \frac{449.7}{138.2} \cdot \frac{14.9}{41.72} \cdot 0.995=0.97
$$

\section{Purity: $\mathbf{9 7 \%}$}




\section{S27. SIMesAgCl, ${ }^{1} \mathrm{H}$ qNMR Vérifié !!}

\begin{tabular}{|l|l|l|l|}
\hline Zero filling & LB & Phase & Baseline \\
\hline $256 \mathrm{~K}$ & 0.1 & Manual & $6^{\mathrm{Th}}$ order \\
\hline
\end{tabular}

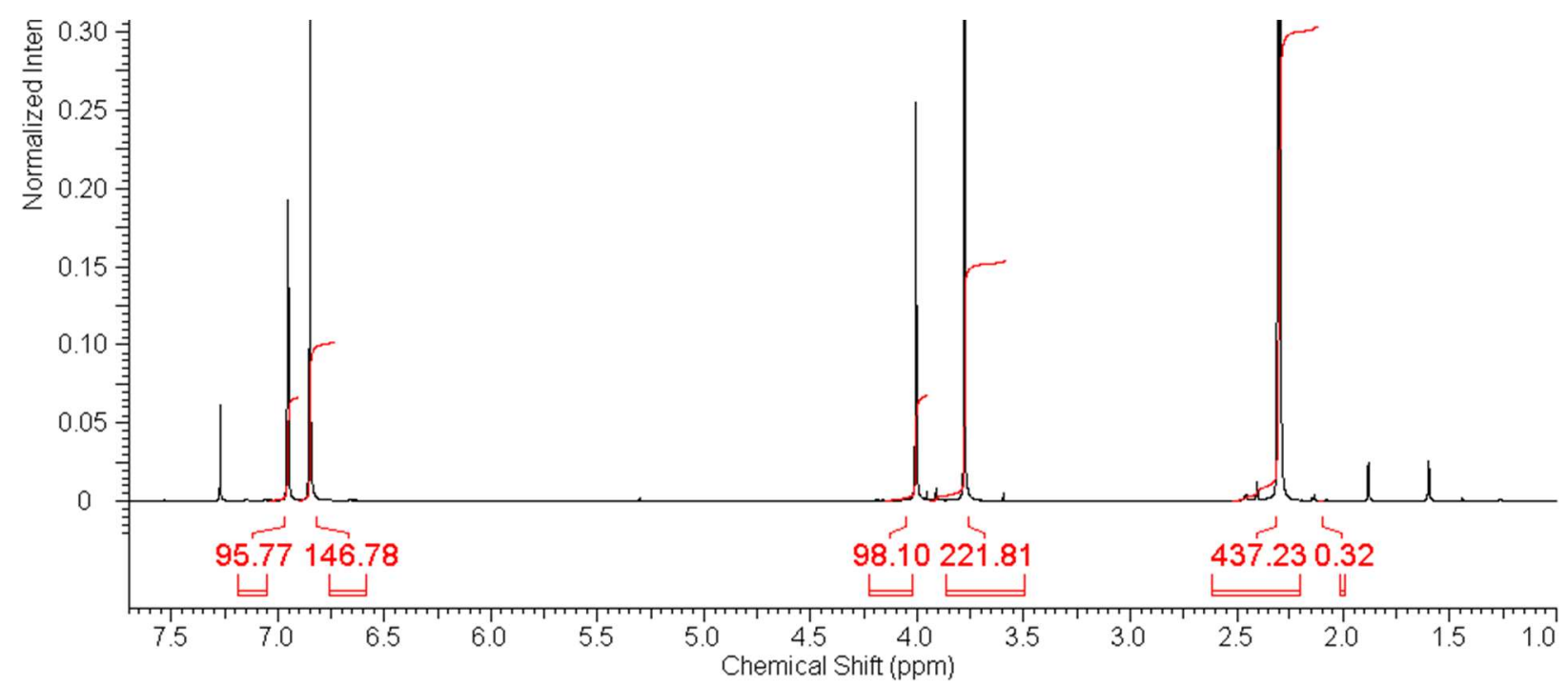

\begin{tabular}{|l|l|l|}
\hline Masse sample $(\mathrm{mg})$ & Masse Standard (mg) & Solvent: $\mathrm{CDCl}_{3}$ \\
\hline 28.79 & 12.74 & \\
\hline
\end{tabular}

\begin{tabular}{|l|l|l|l|}
\hline Signals standard $(\mathrm{ppm})$ & 6.86 & 3.7 & \\
\hline Integration & 146.8 & 221.8 & \\
\hline \hline Signals sample (ppm) & 6.95 & 4 & 2.3 \\
\hline Integration & 95.8 & 98.1 & 437.2 \\
\hline
\end{tabular}

Somme of the integral: sample, $631.1 ;$ standard, 368.6.

Number of protons: sample, 26; standard, 10.

Molecular weight: sample, 449.8; standard, 138.2.

Mass of: sample, 28.79; standard: 12.74 .

$$
P_{s}=\frac{I_{s}}{I_{s t d}} \cdot \frac{N_{s t d}}{N_{s}} \cdot \frac{M_{s}}{M_{s t d}} \cdot \frac{m_{s t d}}{m_{s}} \cdot P_{s t d}=\frac{631.1}{368.7} \cdot \frac{10}{26} \cdot \frac{449.8}{138.2} \cdot \frac{12.74}{28.79} \cdot 0.995=0.95
$$

\section{Purity: 95\%}




\section{S28. IPrAgCl, ${ }^{1} \mathrm{H}$ qNMR Vérifié !}

\begin{tabular}{|l|l|l|l|}
\hline Zero filling & LB & Phase & Baseline \\
\hline $256 \mathrm{~K}$ & 0.1 & Manual & $6^{\text {Th }}$ order \\
\hline
\end{tabular}

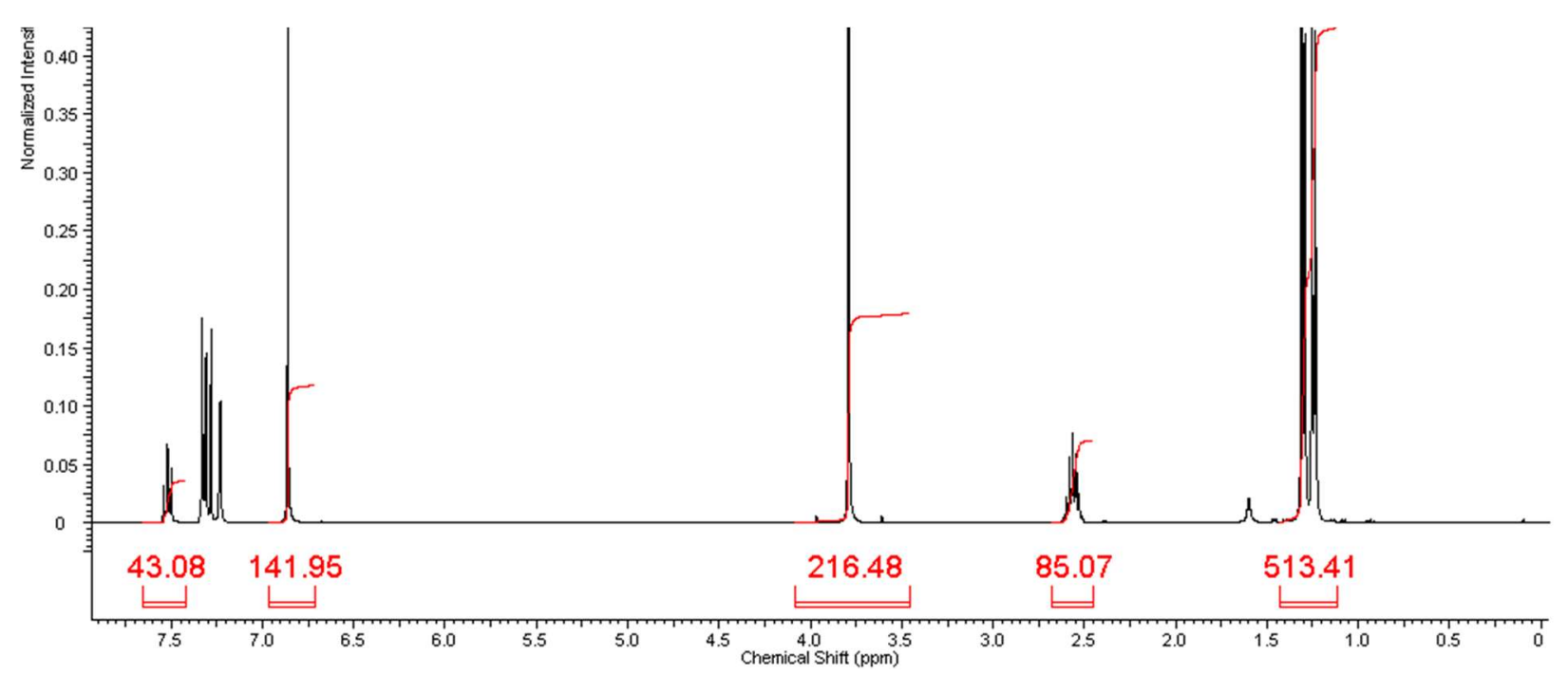

\begin{tabular}{|l|l|l|}
\hline Masse sample $(\mathrm{mg})$ & Masse Standard (mg) & Solvent: $\mathrm{CDCl}_{3}$ \\
\hline 23.89 & 10.20 & \\
\hline
\end{tabular}

\begin{tabular}{|l|l|l|l|}
\hline Signals standard (ppm) & 6.86 & 3.7 & \\
\hline Integration & 142.0 & 216.5 & \\
\hline \hline Signals sample (ppm) & 7.7 & 7.5 & 7.4 \\
\hline Integration & 43.1 & 85.1 & 513.4 \\
\hline
\end{tabular}

Somme of the integral: sample, 641.6; standard, 358.5.

Number of protons: sample, 30; standard, 10.

Molecular weight: sample, 531.9 standard, 138.2.

Mass of: sample, 23.89; standard: 10.2.

$$
P_{s}=\frac{I_{s}}{I_{s t d}} \cdot \frac{N_{s t d}}{N_{s}} \cdot \frac{M_{s}}{M_{s t d}} \cdot \frac{m_{s t d}}{m_{s}} \cdot P_{s t d}=\frac{641.6}{358.5} \cdot \frac{10}{30} \cdot \frac{531.9}{138.2} \cdot \frac{10.2}{23.89} \cdot 0.995=0.97
$$

\section{Purity: $\mathbf{9 7 \%}$}


S29. SIPrAgCl ${ }^{1} \mathrm{H}$ qNMR Vérifié !!

\begin{tabular}{|l|l|l|l|}
\hline Zero filling & LB & Phase & Baseline \\
\hline $256 \mathrm{~K}$ & 0.1 & Manual & $6^{\text {Th }}$ order \\
\hline
\end{tabular}

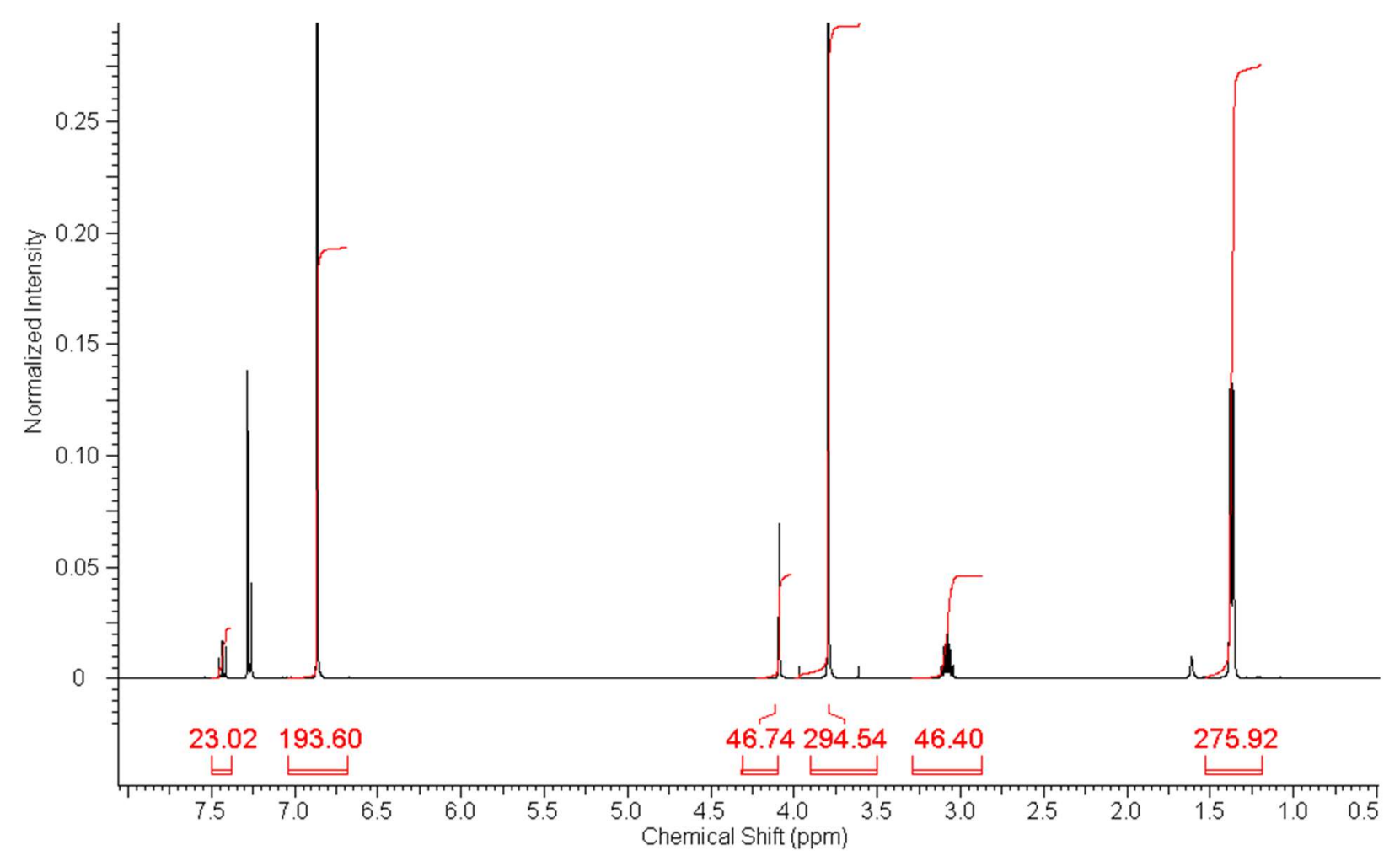

\begin{tabular}{|l|l|l|}
\hline Masse sample (mg) & Masse Standard (mg) & Solvent: $\mathrm{CDCl}_{3}$ \\
\hline 11.86 & 12.83 & \\
\hline
\end{tabular}

\begin{tabular}{|l|l|l|l|l|}
\hline Signals standard (ppm) & 6.86 & 3.7 & & \\
\hline Integration & 193.6 & 294.5 & & \\
\hline \hline Signals sample (ppm) & 7.43 & 4.1 & 3.1 & 1.4 \\
\hline Integration & 23.0 & 46.7 & 46.4 & 275.9 \\
\hline
\end{tabular}

Somme of the integral: sample, 392; standard, 488.1.

Number of protons: sample, 34; standard, 10.

Molecular weight: sample, 533.9; standard, 138.2.

Mass of: sample, 11.86; standard: 12.83.

$$
P_{s}=\frac{I_{s}}{I_{s t d}} \cdot \frac{N_{s t d}}{N_{s}} \cdot \frac{M_{s}}{M_{s t d}} \cdot \frac{m_{s t d}}{m_{s}} \cdot P_{s t d}=\frac{392}{488.1} \cdot \frac{10}{34} \cdot \frac{533.9}{138.2} \cdot \frac{12.83}{11.86} \cdot 0.995=0.98
$$

\section{Purity: $\mathbf{9 8 \%}$}




\section{S30. SIMesCuCl, ${ }^{1} \mathrm{H}$ qNMR Vérifié!!}

\begin{tabular}{|l|l|l|l|}
\hline Zero filling & LB & Phase & Baseline \\
\hline $256 \mathrm{~K}$ & 0.1 & Manual & $6^{\mathrm{Th}}$ order \\
\hline
\end{tabular}

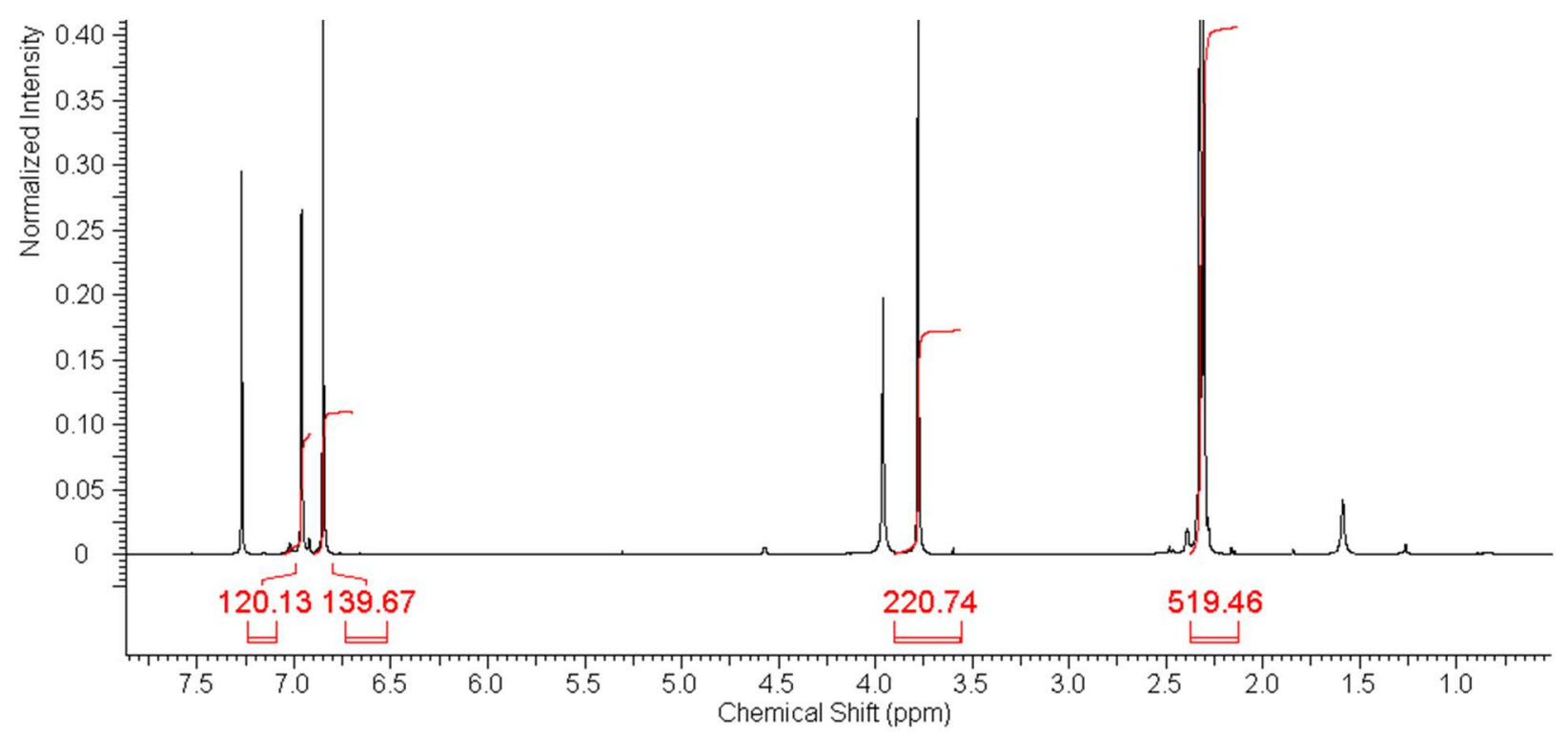

\begin{tabular}{|l|l|l|}
\hline Masse sample (mg) & Masse Standard (mg) & Solvent: CDCl3 \\
\hline 33.25 & 13.18 & \\
\hline
\end{tabular}

\begin{tabular}{|l|l|l|}
\hline Signals standard $(\mathrm{ppm})$ & 6.86 & 3.7 \\
\hline Integration & 139.7 & 220.7 \\
\hline \hline Signals sample (ppm) & 7.0 & 2.3 \\
\hline Integration & 120.1 & 519.5 \\
\hline
\end{tabular}

Somme of the integral: sample, 639.6; standard, 360.4.

Number of protons: sample, 22; standard, 10.

Molecular weight: sample, 405.4; standard, 138.2.

Mass of: sample, 33.25; standard: 13.18 .

$$
P_{s}=\frac{I_{s}}{I_{s t d}} \cdot \frac{N_{s t d}}{N_{s}} \cdot \frac{M_{s}}{M_{s t d}} \cdot \frac{m_{s t d}}{m_{s}} \cdot P_{s t d}=\frac{639.6}{360.4} \cdot \frac{10}{22} \cdot \frac{405.4}{138.2} \cdot \frac{13.18}{33.25} \cdot 0.995=0.93
$$

\section{Purity: 93\%}




\section{S31. IPrCuCl, ${ }^{1} \mathrm{H}$ qNMR VERIFIER !}

\begin{tabular}{|l|l|l|l|}
\hline Zero filling & LB & Phase & Baseline \\
\hline $256 \mathrm{~K}$ & 0.1 & Manual & $6^{\mathrm{Th}}$ order \\
\hline
\end{tabular}

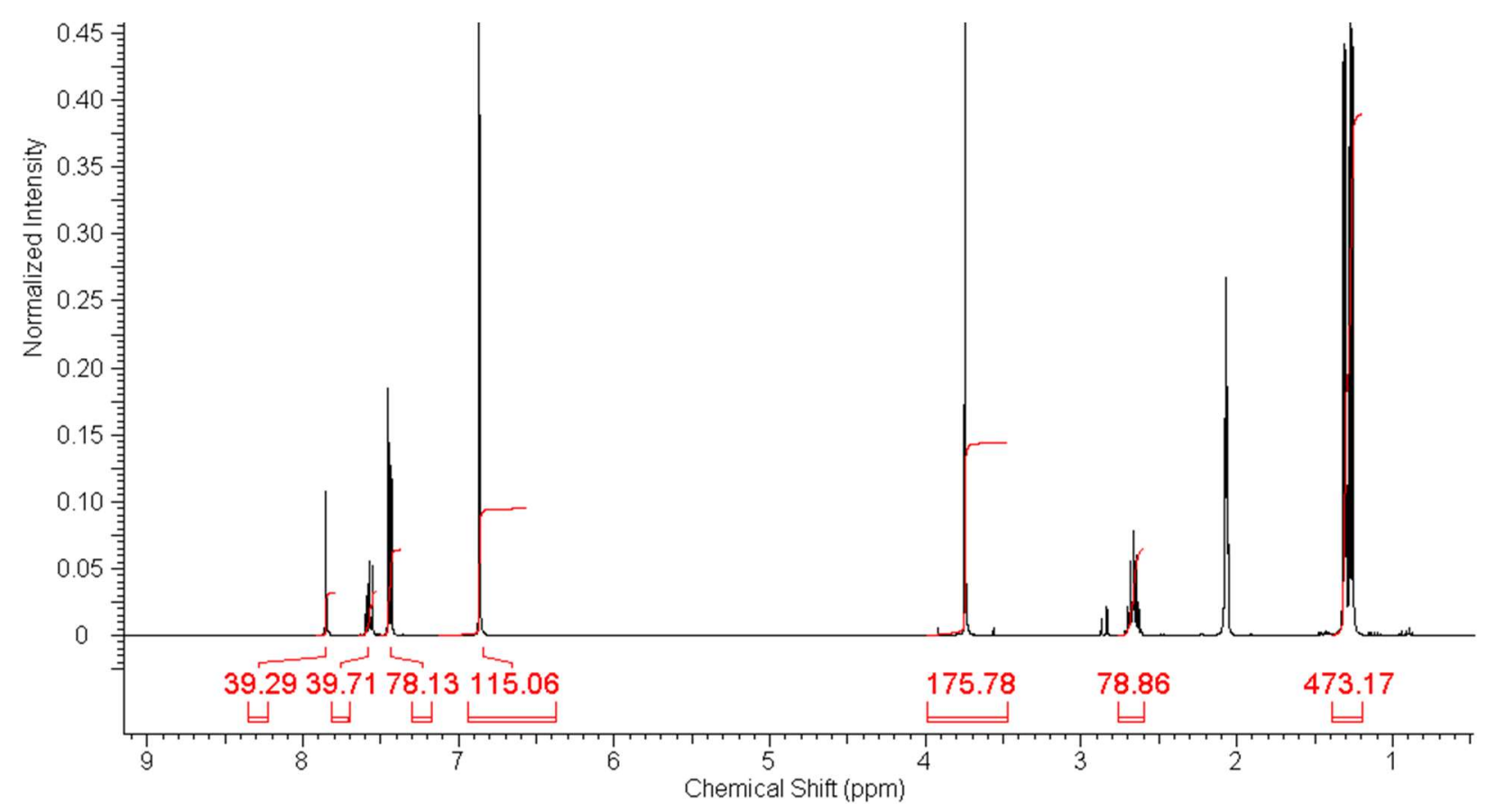

\begin{tabular}{|l|l|l|}
\hline Masse sample $(\mathrm{mg})$ & Masse Standard $(\mathrm{mg})$ & Solvent: Acetone- $\mathrm{d}_{6}$ \\
\hline 31.38 & 13.00 & \\
\hline
\end{tabular}

\begin{tabular}{|l|l|l|l|l|l|}
\hline Signals standard $(\mathrm{ppm})$ & 6.86 & 3.7 & & & \\
\hline Integration & 115.1 & 175.8 & & & \\
\hline \hline Signals sample $(\mathrm{ppm})$ & 7.9 & 7.6 & 7.4 & 2.7 & 1.3 \\
\hline Integration & 39.3 & 39.7 & 78.1 & 78.9 & 473.2 \\
\hline
\end{tabular}

Somme of the integral: sample, 709.2; standard, 290.9.

Number of protons: sample, 36; standard, 10.

Molecular weight: sample, 489.6; standard, 138.2.

Mass of: sample, 31.38; standard: 13.00.

$$
P_{s}=\frac{I_{s}}{I_{s t d}} \cdot \frac{N_{s t d}}{N_{s}} \cdot \frac{M_{s}}{M_{s t d}} \cdot \frac{m_{s t d}}{m_{s}} \cdot P_{s t d}=\frac{709.2}{290.9} \cdot \frac{10}{36} \cdot \frac{489.6}{138.2} \cdot \frac{13.00}{31.38} \cdot 0.995=0.98
$$

\section{Purity: 98\%}




\section{S32. IPrAuCl, ${ }^{1} \mathrm{H}$ qNMR Vérifié !!}

\begin{tabular}{|l|l|l|l|}
\hline Zero filling & LB & Phase & Baseline \\
\hline $256 \mathrm{~K}$ & 0.1 & Manual & $6^{\text {Th }}$ order \\
\hline
\end{tabular}

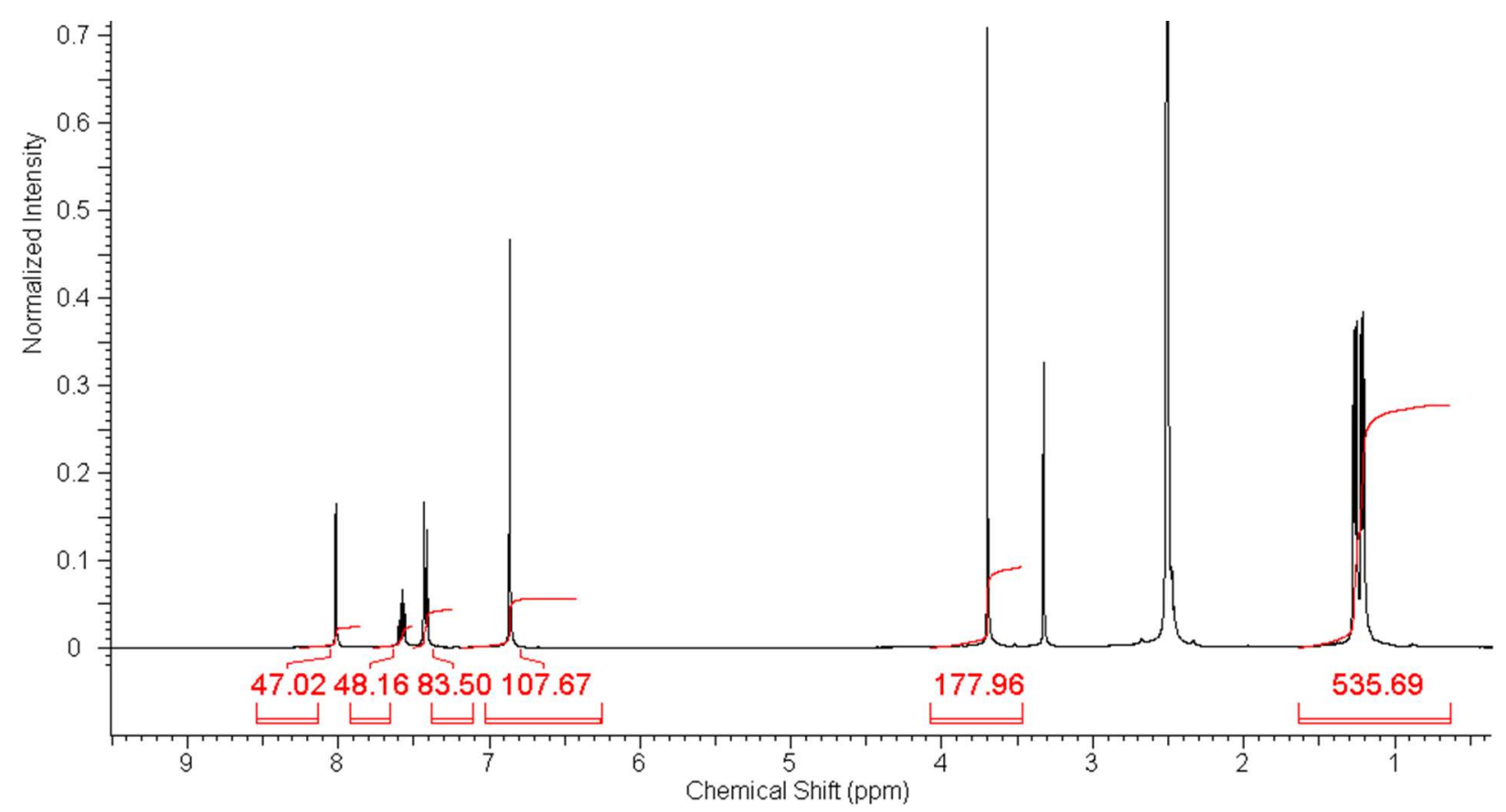

\begin{tabular}{|l|l|l|}
\hline Masse sample (mg) & Masse Standard (mg) & Solvent: dmso- $_{6}$ \\
\hline 50.83 & 13.52 & \\
\hline
\end{tabular}

\begin{tabular}{|l|l|l|l|l|}
\hline Signals standard (ppm) & 6.86 & 3.7 & & \\
\hline Integration & 107.7 & 178.0 & & \\
\hline \hline Signals sample (ppm) & 8.0 & 7.6 & 7.4 & 1.2 \\
\hline Integration & 47 & 48.2 & 83.5 & 535.7 \\
\hline
\end{tabular}

Somme of the integral: sample, 714.4; standard, 285.7.

Number of protons: sample, 32; standard, 10.

Molecular weight: sample, 621; standard, 138.2.

Mass of: sample, 50.83; standard: 13.52.

$$
P_{S}=\frac{I_{s}}{I_{s t d}} \cdot \frac{N_{s t d}}{N_{s}} \cdot \frac{M_{s}}{M_{s t d}} \cdot \frac{m_{s t d}}{m_{s}} \cdot P_{s t d}=\frac{714.4}{285.7} \cdot \frac{10}{32} \cdot \frac{621.0}{138.2} \cdot \frac{13.52}{50.83} \cdot 0.995=0.92
$$

\section{Purity: 92\%}




\section{S33. [IPrAu,MeCN], $\mathrm{BF}_{4},{ }^{1} \mathrm{H}$ qNMR, Vérifié!!}

\begin{tabular}{|l|l|l|l|}
\hline Zero filling & LB & Phase & Baseline \\
\hline $256 \mathrm{~K}$ & 0.1 & Manual & $6^{\mathrm{Th}}$ order \\
\hline
\end{tabular}

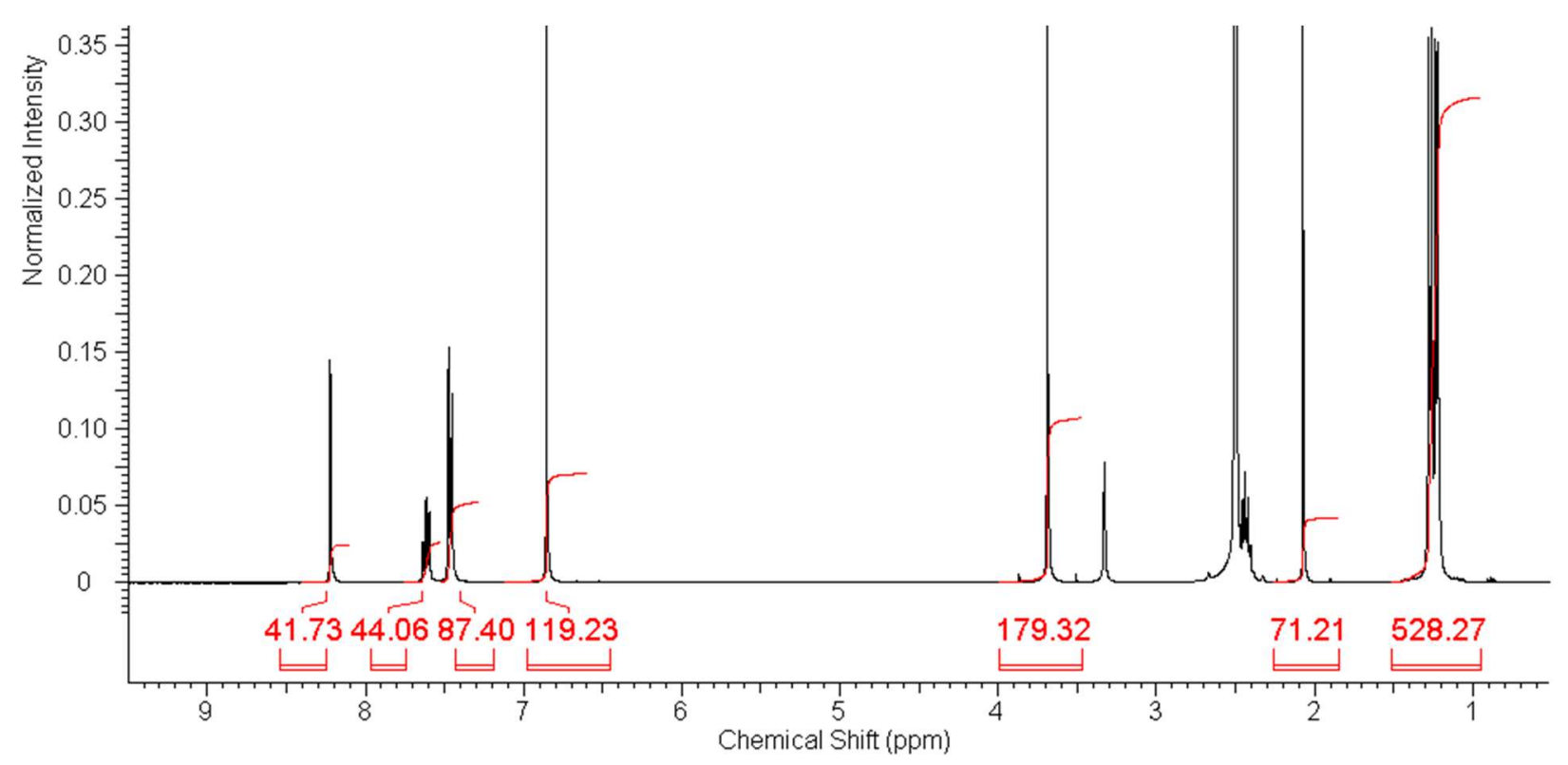

\begin{tabular}{|l|l|l|}
\hline Masse sample (mg) & Masse Standard (mg) & Solvent: dmso- $\mathrm{d}_{6}$ \\
\hline 49.3 & 12.21 & \\
\hline
\end{tabular}

\begin{tabular}{|l|l|l|l|l|l|}
\hline Signals standard (ppm) & 6.86 & 3.7 & & & \\
\hline Integration & 119.2 & 179.3 & & & \\
\hline \hline Signals sample (ppm) & 8.2 & 7.6 & 7.5 & 2.0 & 1.3 \\
\hline Integration & 41.7 & 44.1 & 87.4 & 71.2 & 528.3 \\
\hline
\end{tabular}

Somme of the integral: sample, 772.71; standard, 289.5 .

Number of protons: sample, 35; standard, 10.

Molecular weight: sample, 713.4; standard, 138.2.

Mass of: sample, 49.3 ; standard: 12.21.

$$
P_{s}=\frac{I_{s}}{I_{s t d}} \cdot \frac{N_{s t d}}{N_{s}} \cdot \frac{M_{s}}{M_{s t d}} \cdot \frac{m_{s t d}}{m_{s}} \cdot P_{s t d}=\frac{772.7}{289.5} \cdot \frac{10}{35} \cdot \frac{713.4}{138.2} \cdot \frac{12.21}{49.3} \cdot 0.995=0.93
$$

\section{Purity: 93\%}




\section{S34. [IPrAu, MeCN], $\mathrm{BF}_{4},{ }^{19} \mathrm{~F}$ qNMR Verifié}

\begin{tabular}{|l|l|l|l|}
\hline Zero filling & LB & Phase & Baseline \\
\hline $256 \mathrm{~K}$ & 0.1 & Manual & FID reconstruction \\
\hline
\end{tabular}

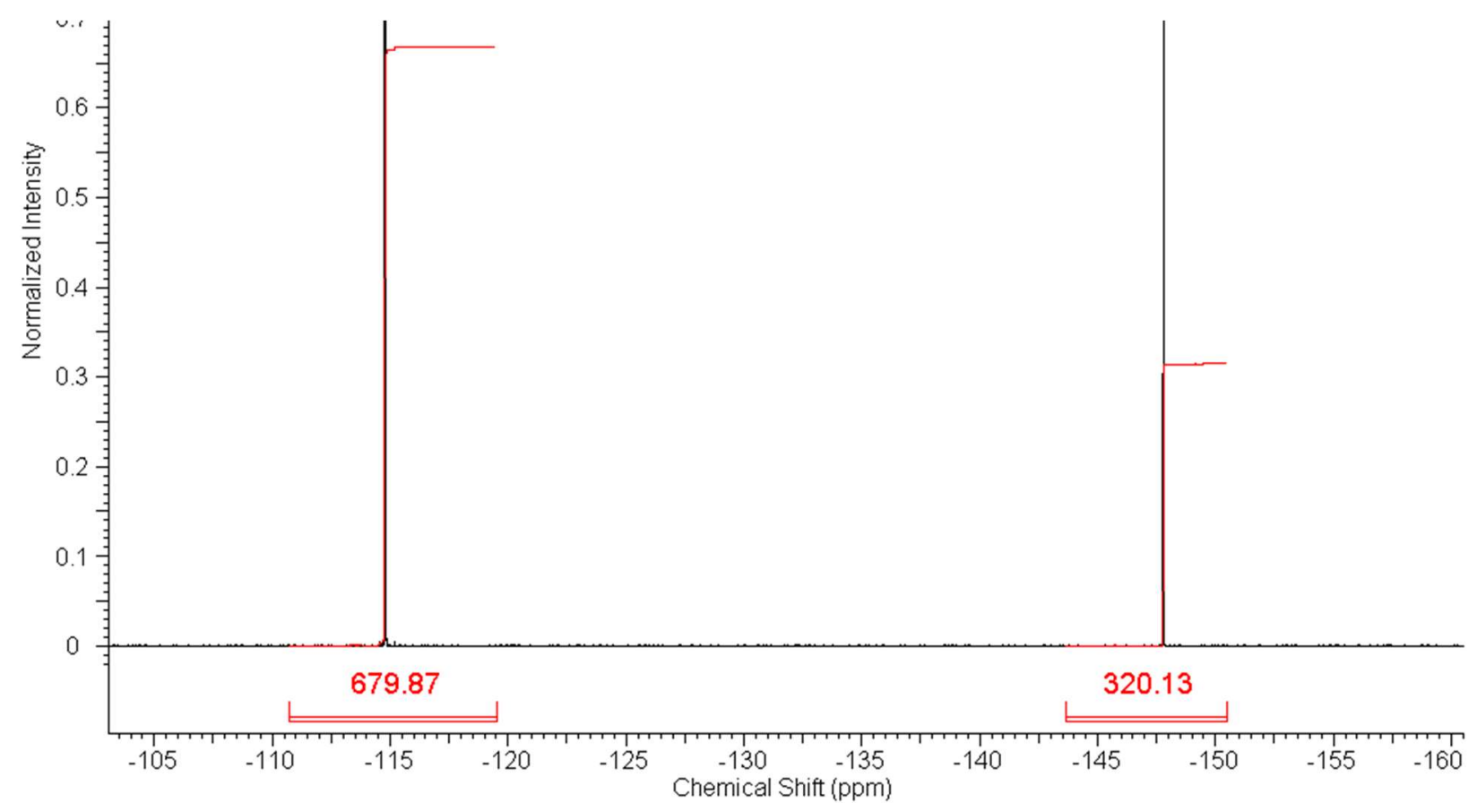

\begin{tabular}{|l|l|l|}
\hline Masse sample $(\mathrm{mg})$ & Masse Standard $(\mathrm{mg})$ & Solvent: dmso- $\mathrm{d}_{6}$ \\
\hline 16.74 & 26.74 & \\
\hline
\end{tabular}

\begin{tabular}{|l|l|}
\hline Signals standard $(\mathrm{ppm})$ & -115 \\
\hline Integration & 679.9 \\
\hline \hline Signals sample (ppm) & -148 \\
\hline Integration & 320.1 \\
\hline
\end{tabular}

Somme of the integral: sample, 320.1; standard, 679.9.

Number of fluorine: sample, 4; standard, 1.

Molecular weight: sample, 713.4; standard, 144.6.

Mass of: sample, $16.74 ;$ standard: 26.74.

$$
P_{s}=\frac{I_{s}}{I_{s t d}} \cdot \frac{N_{s t d}}{N_{s}} \cdot \frac{M_{s}}{M_{s t d}} \cdot \frac{m_{s t d}}{m_{s}} \cdot P_{s t}=\frac{320.1}{679.9} \cdot \frac{1}{4} \cdot \frac{713.4}{144.6} \cdot \frac{26.74}{16.74} \cdot 0.996=0.95
$$

\section{Purity: $95 \%$}




\section{S35. PEPSSI. 0.8 $\mathrm{CH}_{2} \mathrm{Cl}_{2},{ }^{1} \mathrm{H}$ qNMR Verifié!!}

\begin{tabular}{|l|l|l|l|}
\hline Zero filling & LB & Phase & Baseline \\
\hline $256 \mathrm{~K}$ & 0.1 & Manual & $6^{\text {Th }}$ order \\
\hline
\end{tabular}

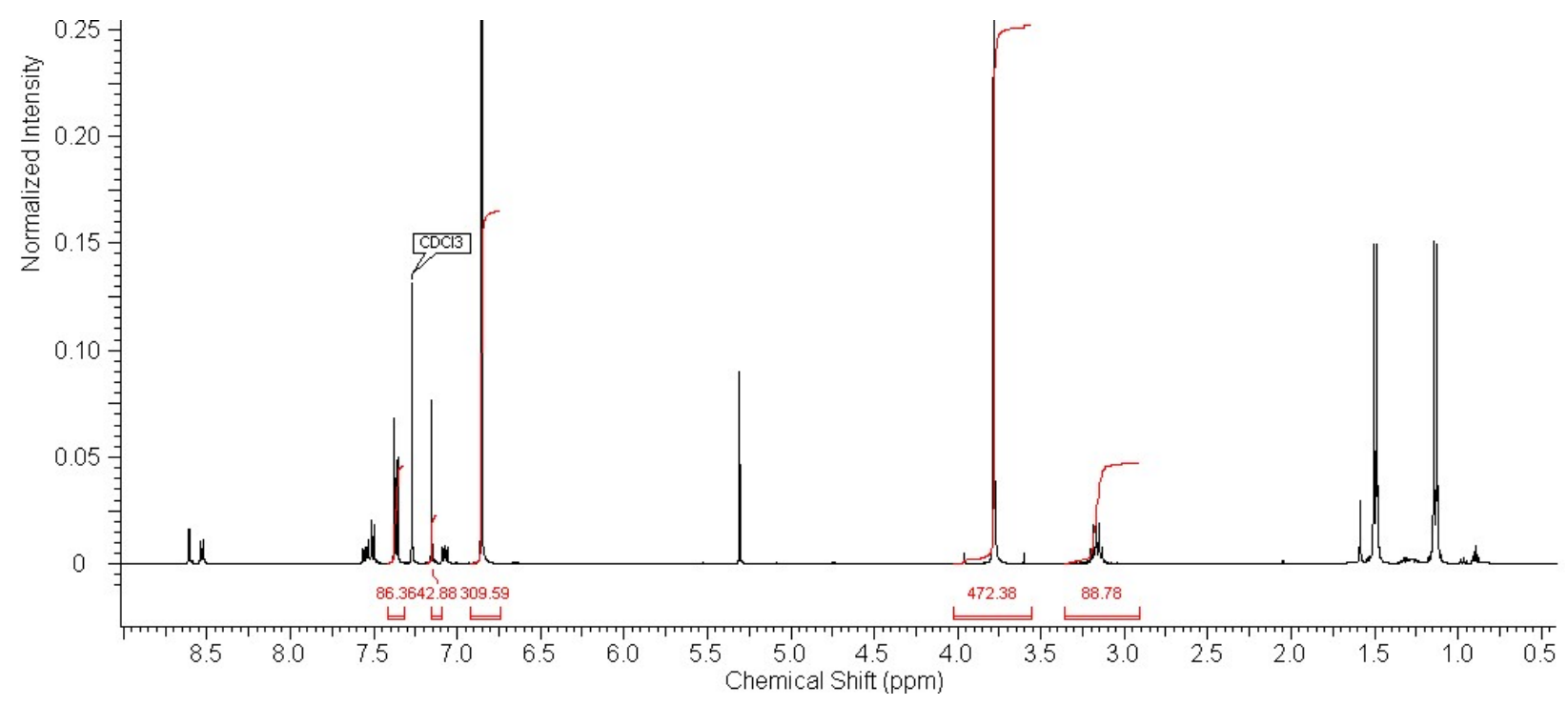

\begin{tabular}{|l|l|l|}
\hline Masse sample (mg) & Masse Standard (mg) & Solvent: $\mathrm{CDCl}_{3}$ \\
\hline 19.73 & 13.43 & \\
\hline
\end{tabular}

\begin{tabular}{|l|l|l|l|}
\hline Signals standard (ppm) & 6.7 & 3.7 & \\
\hline Integration & 309.6 & 472.4 & \\
\hline \hline Signals sample (ppm) & 7.37 & 7.15 & 3.16 \\
\hline Integration & 86.4 & 42.9 & 88.8 \\
\hline
\end{tabular}

Somme of the integral: sample, 307.1; standard, 782.

Number of protons: sample, 14; standard, 10.

Molecular weight: sample, 745; standard, 138.2.

Mass of: sample (0.8 dcm), 19.73; standard: 12.43 .

$$
P_{s}=\frac{I_{s}}{I_{s t d}} \cdot \frac{N_{s t d}}{N_{s}} \cdot \frac{M_{s}}{M_{s t d}} \cdot \frac{m_{s t d}}{m_{s}} \cdot P_{s t d}=\frac{307}{782} \cdot \frac{10}{14} \cdot \frac{745}{138.2} \cdot \frac{12.43}{19.73} \cdot 0.995=0.96
$$

\section{Purity: 96\%}

